\title{
Catalytic Four-component Assembly Based on Allenylboronate Platform: A New Access to Privileged Allylic Amine Structures
}

\author{
Keisuke Tonogaki, ${ }^{\dagger}$ Kenichiro Itami,,${ }^{, *}$ and Jun-ichi Yoshida ${ }^{\dagger, *}$ \\ 'Department of Synthetic Chemistry \& Biological Chemistry, Graduate School of Engineering, \\ Kyoto University, Nishikyo-ku, Kyoto 615-8510, Japan \\ ${ }^{\ddagger}$ Research Center for Materials Science, Nagoya University, Nagoya 464-8602, Japan
}

\section{General.}

${ }^{1} \mathrm{H}$ and ${ }^{13} \mathrm{C}$ NMR spectra were recorded on Varian MERCURY plus-400 $\left({ }^{1} \mathrm{H} 400 \mathrm{MHz},{ }^{13} \mathrm{C} 100 \mathrm{MHz}\right)$ spectrometers in $\mathrm{CDCl}_{3}$ using tetramethylsilane $\left({ }^{1} \mathrm{H}, \delta=0.00\right)$ and $\mathrm{CDCl}_{3}\left({ }^{13} \mathrm{C}, \delta=77.0\right)$ as internal standards. EI mass spectra were recorded on JMS-SX102A spectrometer. FAB mass spectra were recorded on JMS-HX110A spectrometer. Gel permeation chromatography was carried out with Japan Analytical Industry LC-918. Column chromatography was performed with silica gel 60 A (SILICYCLE). Unless otherwise noted, all reactions were carried out under argon atmosphere. Unless otherwise noted, all materials were obtained from commercial suppliers and used without further purification. All amines were used after distillation from $\mathrm{CaH}_{2}$. Phenylallene, ${ }^{1}$ cyclohexylallene, ${ }^{1}$ and triisopropylsilylallene ${ }^{2}$ were prepared by the literature procedures.

\section{Synthesis of Allenylboronate Pinacol Ester (1).}

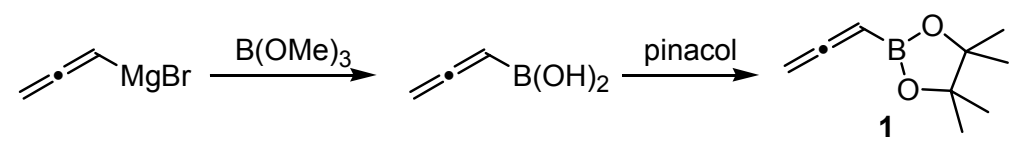

Freshly distilled trimethylborate $(14.5 \mathrm{~g}, 140 \mathrm{mmol})$ was added to a round bottom flask containing ether $(105 \mathrm{~mL})$ and cooled to $-78{ }^{\circ} \mathrm{C}$. A $0{ }^{\circ} \mathrm{C}$ solution of allenylmagnesium bromide ${ }^{3}(1 \mathrm{M}$ in ether, 140 mmol) was added via canula at rate as to keep the internal reaction temperature below $-65{ }^{\circ} \mathrm{C}$. The resultant mixture was stirred at $-78{ }^{\circ} \mathrm{C}$ for $3 \mathrm{~h}$, warmed to $0{ }^{\circ} \mathrm{C}$ over $1 \mathrm{~h}$, and stirred at $0{ }^{\circ} \mathrm{C}$ for an additional hour. Aqueous $\mathrm{HCl}(2 \mathrm{M}, 280 \mathrm{mmol})$ was added via an addition funnel to form the boronic acid. After the mixture was stirred for $1 \mathrm{~h}$, the organic layer was separated from the aqueous layer. The aqueous layer was extracted with ether and combined organic layers were dried over $\mathrm{Na}_{2} \mathrm{SO}_{4}$. The filtrate was evaporated until $\sim 180 \mathrm{~mL}$ of solvent remained (rotary evaporator was flushed and backfilled using an argon balloon). Pinacol (11.8 g, $100 \mathrm{mmol})$ and $\mathrm{MgSO}_{4}(20 \mathrm{~g}, 166 \mathrm{mmol})$ were added to the flask and the mixture was stirred for $14 \mathrm{~h}$ at room temperature. The silica gel chromatography of the residue (pentane/ether $=10 / 1)$ followed by distillation $\left(\mathrm{bp} 80-81{ }^{\circ} \mathrm{C} / 18.6 \mathrm{mmHg}\right.$,) afforded $1(7.6 \mathrm{~g}$, $46 \%)$ as a colorless oil: ${ }^{1} \mathrm{H}$ NMR $\left(400 \mathrm{MHz}, \mathrm{CDCl}_{3}\right) \delta 1.28(\mathrm{~s}, 12 \mathrm{H}), 4.65(\mathrm{~d}, J=7.2 \mathrm{~Hz}, 2 \mathrm{H}), 4.91(\mathrm{t}, J=7.2$ $\mathrm{Hz}, 1 \mathrm{H}) .{ }^{13} \mathrm{C}$ NMR $\left(100 \mathrm{MHz}, \mathrm{CDCl}_{3}\right) \delta 24.8,70.0$, 83.7, 218.6. HRMS (EI) $\mathrm{m} / z$ calcd for $\mathrm{C}_{9} \mathrm{H}_{15} \mathrm{BO}_{2}$ : 166.1165, found 166.1168 . 
Procedure for Three-component Assembly Reaction of Allenylboronate Pinacol Ester, Benzylamine, and 4-Iodotoluene (Eq 1).

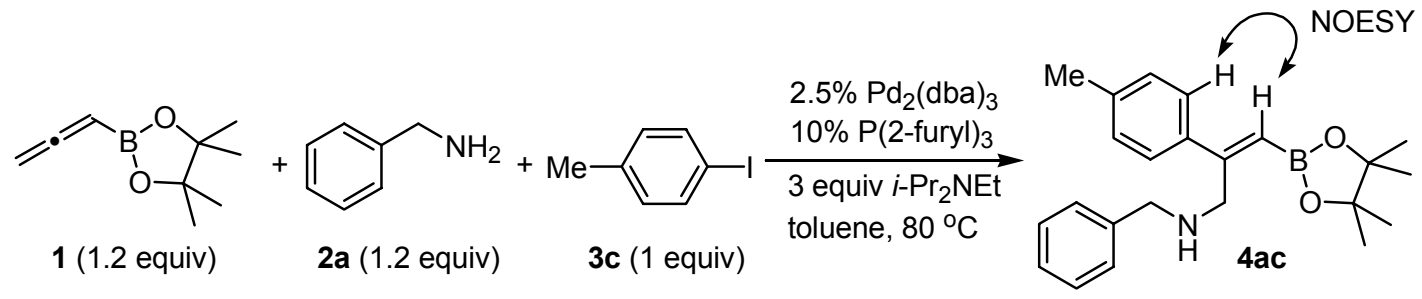

A mixture of $\mathrm{Pd}_{2}(\mathrm{dba})_{3}(6.9 \mathrm{mg}, 7.5 \mu \mathrm{mol}, 2.5 \mathrm{~mol} \%)$ and $\mathrm{P}(2$-furyl $) 3(7.0 \mathrm{mg}, 30 \mu \mathrm{mol}, 10 \mathrm{~mol} \%)$ in dry toluene $(0.5 \mathrm{~mL})$ was stirred at room temperature for $20 \mathrm{~min}$ under argon. To this mixture were added 4-iodotoluene (3c; $65.6 \mathrm{mg}, 0.30 \mathrm{mmol})$, allenylboronate pinacol ester (1; $59.8 \mathrm{mg}, 0.36 \mathrm{mmol})$, benzylamine (2a; $38.6 \mathrm{mg}, 0.36 \mathrm{mmol}), i$-Pr2 NEt $(116.3 \mathrm{mg}, 0.90 \mathrm{mmol})$, and dry toluene $(1.0 \mathrm{~mL})$ and the resultant mixture was further stirred at $80{ }^{\circ} \mathrm{C}$ for $24 \mathrm{~h}$. After cooling the reaction mixture to room temperature, catalyst and salts were removed by filtration (toluene). The filtrate was evaporated to afford crude product. The yield of 4 ac was determined to be $>99 \%$ from the ${ }^{1} \mathrm{H}$ NMR analysis of crude mixture using internal standard (tetrachloroethane). The purification of the residue by gel permeation chromatography $\left(\mathrm{CHCl}_{3}\right)$ afforded 4 ac $(78.3 \mathrm{mg}, 71 \%)$ as yellow solid: ${ }^{1} \mathrm{H} \mathrm{NMR}\left(400 \mathrm{MHz}, \mathrm{CDCl}_{3}\right) \delta 1.27$ $(\mathrm{s}, 12 \mathrm{H}), 2.30(\mathrm{~s}, 3 \mathrm{H}), 3.68(\mathrm{~s}, 2 \mathrm{H}), 4.00(\mathrm{~s}, 2 \mathrm{H}), 6.33(\mathrm{~s}, 1 \mathrm{H}), 7.06(\mathrm{~d}, J=8.0 \mathrm{~Hz}, 2 \mathrm{H}), 7.20(\mathrm{~d}, J=8.0 \mathrm{~Hz}$, $2 \mathrm{H}), 7.28(\mathrm{dd}, J=7.0,2.0 \mathrm{~Hz}, 2 \mathrm{H}), 7.35-7.43(\mathrm{~m}, 3 \mathrm{H}) .{ }^{13} \mathrm{C} \mathrm{NMR}\left(100 \mathrm{MHz}, \mathrm{CDCl}_{3}\right) \delta 21.2,25.5,52.3,52.7$, 79.7, 124.8, 128.3, 128.4, 128.7, 129.0, 134.0, 135.6, 136.9, 142.1. HRMS (EI) $\mathrm{m} / \mathrm{z}$ calcd for $\mathrm{C}_{23} \mathrm{H}_{30} \mathrm{BNO}_{2}$ : 363.2370, found 363.2372 .

\section{Effect of Phosphine Ligand on Palladium for Three-component Assembly Reaction. ${ }^{a}$}

Table S1.

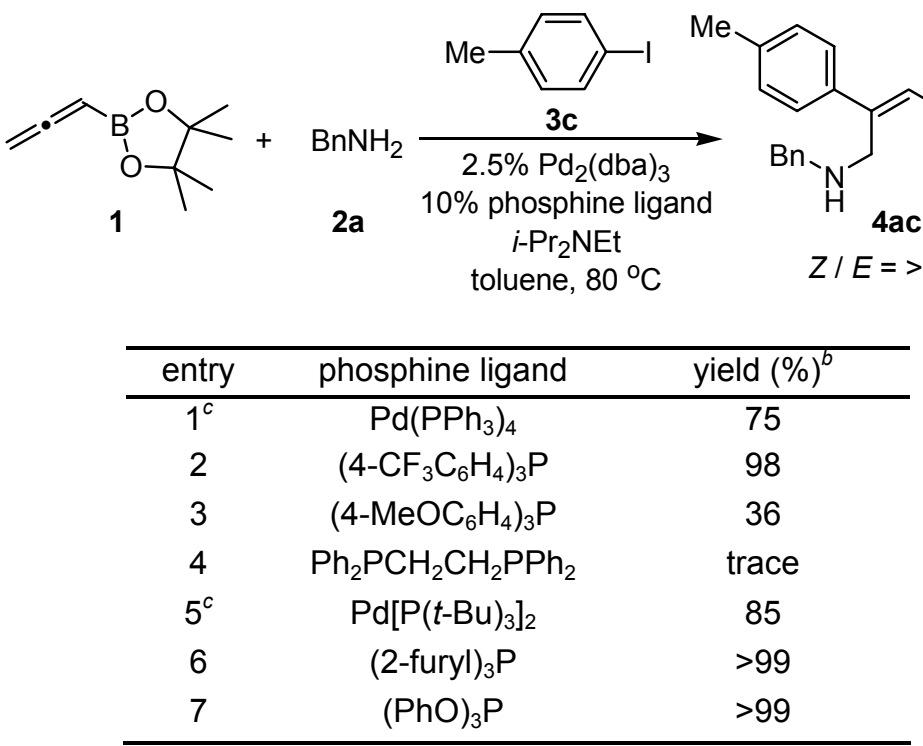

${ }^{a}$ Reaction conditions: 1 (0.36 mmol), 3c (0.30 mmol), $\mathrm{Pd}_{2}(\mathrm{dba})_{3}(2.5 \mathrm{~mol} \%)$, phosphine ligand (10 mol \%), $2 \mathrm{a}(0.36 \mathrm{mmol}), i$-Pr $2 \mathrm{NEt}(0.90 \mathrm{mmol})$, toluene $(1.5 \mathrm{~mL}), 80{ }^{\circ} \mathrm{C}, 24 \mathrm{~h} .{ }^{b} \mathrm{NMR}$ yield. ${ }^{c}$ Palladium catalysts were employed instead. 
Synthesis, Structure, and Reaction of ( $\pi$-allyl)Pd Complex 7 (Eq 2).
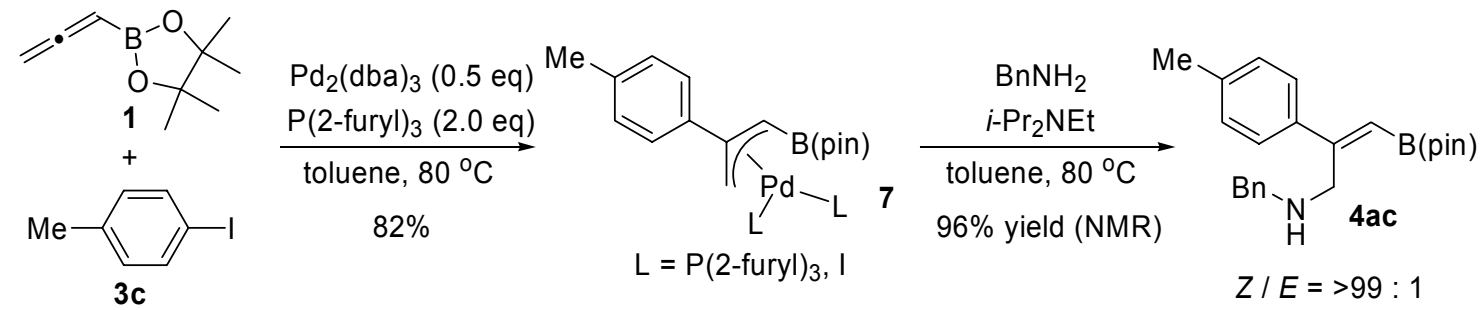

Synthsis of ( $\pi$-allyl)Pd Complex 7: A mixture of $\mathrm{Pd}_{2}(\mathrm{dba})_{3}(137.4 \mathrm{mg}, 0.15 \mathrm{mmol})$ and $\mathrm{P}(2 \text {-furyl })_{3}$ $(139.0 \mathrm{mg}, 0.60 \mathrm{mmol})$ in dry toluene $(2.0 \mathrm{~mL})$ was stirred at room temperature for $20 \mathrm{~min}$ under argon. To this mixture were added 4-iodotoluene (3c; $72.0 \mathrm{mg}, 0.33 \mathrm{mmol})$, allenylboronate pinacol ester (1; $54.9 \mathrm{mg}, 0.33 \mathrm{mmol})$, and dry toluene $(0.5 \mathrm{~mL})$ and the resultant mixture was further stirred at $80{ }^{\circ} \mathrm{C}$ for $12 \mathrm{~h}$. After cooling the reaction mixture to room temperature, the residue was subjected to gel permeation chromatography $\left(\mathrm{CHCl}_{3}\right)$ to afford $7(178.2 \mathrm{mg}, 82 \%)$ as a mixture of two isomers (major/minor = 67/33). ${ }^{1} \mathrm{H}$ NMR $\left(400 \mathrm{MHz}, \mathrm{CDCl}_{3}\right) \delta 1.02(\mathrm{~s}, 6 \mathrm{H}$, minor isomer), $1.12(\mathrm{~s}, 6 \mathrm{H}$, minor isomer), $1.29(\mathrm{~s}, 6 \mathrm{H}$, major isomer), $1.30(\mathrm{~s}, 6 \mathrm{H}$, major isomer), $2.34(\mathrm{~s}, 6 \mathrm{H}), 4.09(\mathrm{dd}, J=2.0,0.4 \mathrm{~Hz}, 1 \mathrm{H}$, major isomer), $4.32(\mathrm{dd}, J=11.2,1.2 \mathrm{~Hz}, 1 \mathrm{H}$, minor isomer), $4.49(\mathrm{dd}, J=2.8,2.0 \mathrm{~Hz}, 1 \mathrm{H}$, major isomer), $4.65(\mathrm{~d}, J=3.2 \mathrm{~Hz}, 1 \mathrm{H}$, minor isomer), $4.84(\mathrm{dd}, J=7.6,2.8 \mathrm{~Hz}, 1 \mathrm{H}$, major isomer), 5.10 (ddd, $J=6.8,2.8$, $1.2 \mathrm{~Hz}, 1 \mathrm{H}$, minor isomer), 6.41-6.45 (m, $6 \mathrm{H}), 7.02-7.04(\mathrm{~m}, 3 \mathrm{H}$, major isomer), 7.06-7.08 $(\mathrm{m}, 3 \mathrm{H}$, minor isomer), $7.10(\mathrm{~d}, J=8.0 \mathrm{~Hz}, 2 \mathrm{H}$, major isomer), $7.11(\mathrm{~d}, J=8.0 \mathrm{~Hz}, 2 \mathrm{H}$, minor isomer), $7.30(\mathrm{~d}, J=8.0 \mathrm{~Hz}$, $2 \mathrm{H}$, major isomer), $7.36(\mathrm{~d}, J=8.0 \mathrm{~Hz}, 2 \mathrm{H}$, minor isomer $), 7.65-7.66(\mathrm{~m}, 6 \mathrm{H}) .{ }^{13} \mathrm{C} \mathrm{NMR}\left(100 \mathrm{MHz}, \mathrm{CDCl}_{3}\right)$ $\delta 21.3$ (major isomer), 24.4 (minor isomer), 24.9 (major isomer), 24.96 (minor isomer), 25.02, 66.68 (major isomer), 67.70 (minor isomer), 72.9 (major isomer), 73.3 (minor isomer), 83.2 (minor isomer), 83.5 (major isomer), 110.9 (JC-P $=8.3 \mathrm{~Hz}$, minor isomer), $111.0\left(J_{\mathrm{C}-\mathrm{P}}=8.3 \mathrm{~Hz}\right.$, major isomer $), 124.3$ (JC-P $=23.0 \mathrm{~Hz}$, major isomer), 124.8 (JC-P $=21.9 \mathrm{~Hz}$, minor isomer), 126.5 (major isomer), 126.6 (minor isomer), 129.0 (minor isomer), 129.1 (major isomer), 135.8 (major isomer), 136.1 (minor isomer), 138.51 (JC-P = $5.5 \mathrm{~Hz}$, major isomer), 138.54 (JC-P $=4.8 \mathrm{~Hz}$, minor isomer), 139.3 (minor isomer), 139.4 (major isomer), 143.9 (JC-P $=67.0 \mathrm{~Hz}$, minor isomer), 144.1 (JC-p $=66.7 \mathrm{~Hz}$, major isomer), 147.9 (JC-P $=5.1 \mathrm{~Hz}$, minor isomer), $148.1\left(\mathrm{Jc}-\mathrm{P}=4.8 \mathrm{~Hz}\right.$, major isomer). HRMS (FAB) $\mathrm{m} / z$ calcd for $\mathrm{C}_{28} \mathrm{H}_{31} \mathrm{BO}_{5} \mathrm{PPd}\left([\mathrm{M}-\mathrm{I}]^{+}\right): 595.1037$, found 595.1046.

From the NOE experiments, the structures of these two isomers were determined as shown below.
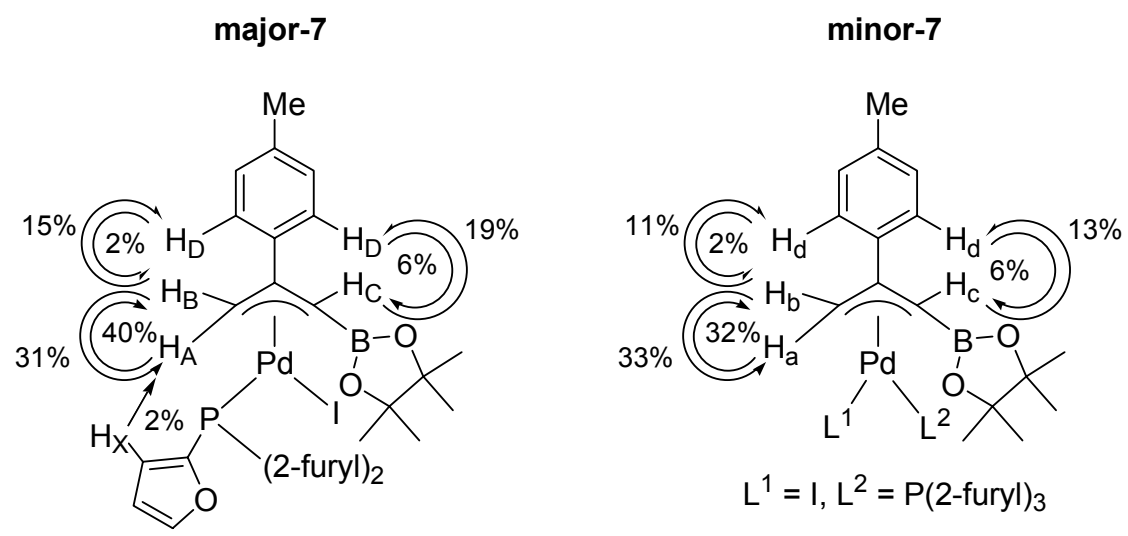

Reaction of ( $\pi$-allyl)Pd Complex 7 with Benzylamine (2a): A mixture of 7 (85.2 $\mathrm{mg}, 0.11 \mathrm{mmol}$ ), benzylamine (2a; $15.6 \mathrm{mg}, 0.15 \mathrm{mmol})$, and $i-\operatorname{Pr}_{2} \mathrm{NEt}(47.0 \mathrm{mg}, 0.36 \mathrm{mmol})$ in dry toluene $(2.0 \mathrm{~mL})$ was stirred at $80^{\circ} \mathrm{C}$ for $12 \mathrm{~h}$. After cooling the reaction mixture to room temperature, insoluble salts were removed by filtration (toluene). The filtrate was evaporated to afford crude product. The yield of 4ac was determined to be $96 \%$ from the ${ }^{1} \mathrm{H}$ NMR analysis of crude mixture using internal standard (tetrachloroethane). 
Effect of Boryl Group in Three-component Assembly Reaction. ${ }^{a}$

Table S2.
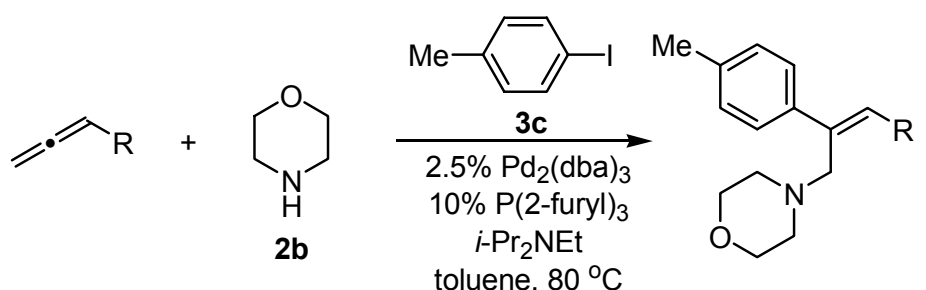

$2 b$

toluene, $80^{\circ} \mathrm{C}$

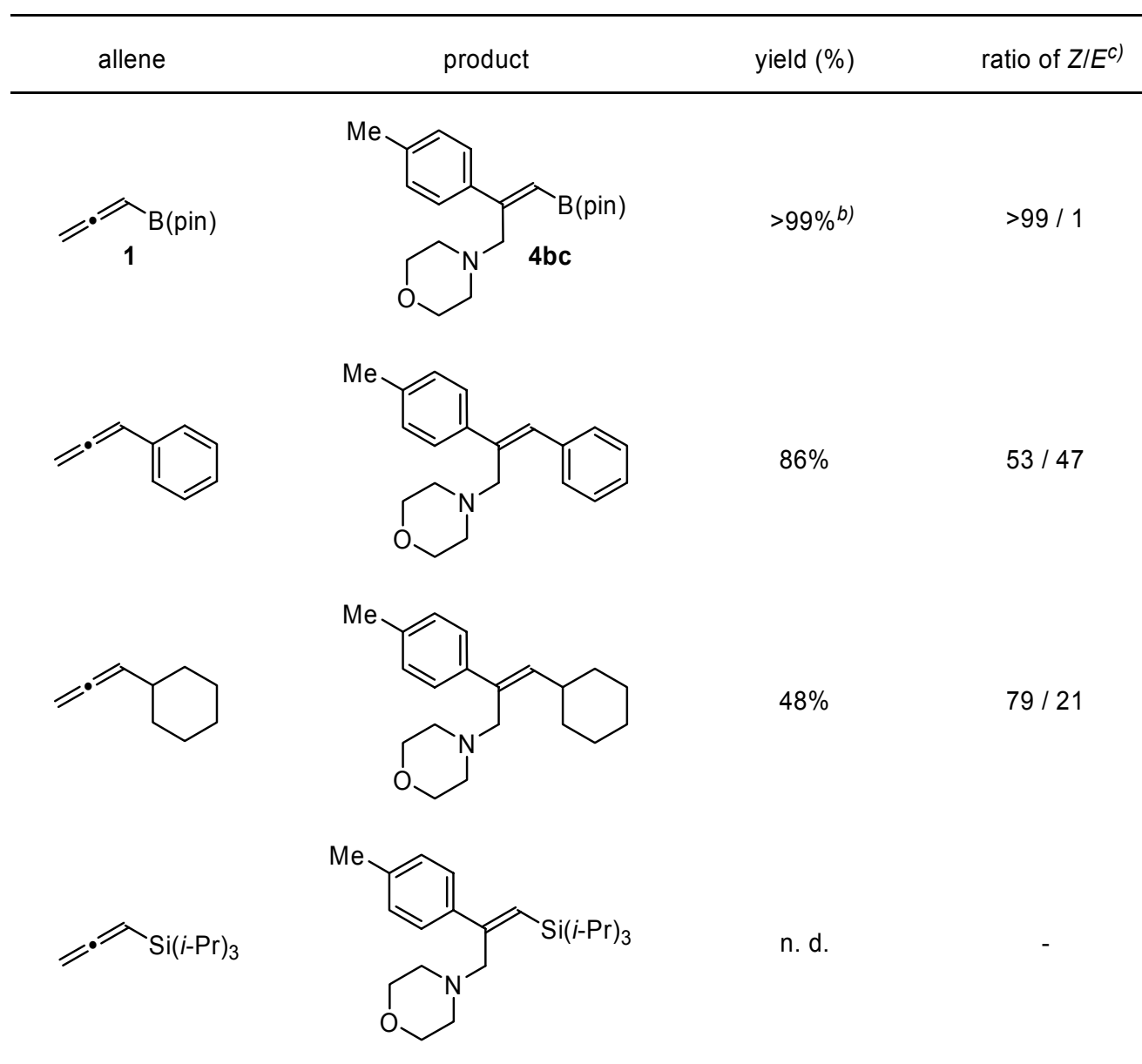

${ }^{a}$ Reaction conditions: Allene $(0.36 \mathrm{mmol}), 3 \mathbf{c}(0.30 \mathrm{mmol}), \mathrm{Pd}_{2}(\mathrm{dba})_{3}(2.5 \mathrm{~mol} \%), \mathrm{P}(2 \text {-furyl })_{3}(10$ mol \%), $2 \mathbf{b}(0.36 \mathrm{mmol}), i$-Pr 2 NEt $(0.90 \mathrm{mmol})$, toluene $(1.5 \mathrm{~mL}), 80^{\circ} \mathrm{C}, 24 \mathrm{~h} .{ }^{b}$ NMR yield. ${ }^{c}$ The ratio of $Z / E$ was determined by ${ }^{1} \mathrm{H}$ NMR analysis. 


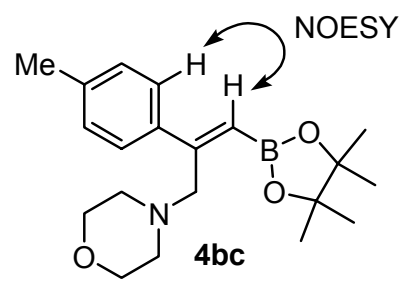

4bc: ${ }^{1} \mathrm{H}$ NMR (400 MHz, CDCl$) \delta 1.29(\mathrm{~s}, 12 \mathrm{H}), 2.33(\mathrm{~s}, 3 \mathrm{H}), 2.76(\mathrm{t}, J=4.8 \mathrm{~Hz}, 4 \mathrm{H}), 3.74(\mathrm{t}, J=4.8 \mathrm{~Hz}$, $4 \mathrm{H}), 3.80(\mathrm{~s}, 2 \mathrm{H}), 6.06(\mathrm{~s}, 1 \mathrm{H}), 7.10(\mathrm{~d}, J=8.0 \mathrm{~Hz}, 2 \mathrm{H}), 7.37(\mathrm{~d}, J=8.0 \mathrm{~Hz}, 2 \mathrm{H}) .{ }^{13} \mathrm{C} \mathrm{NMR}\left(100 \mathrm{MHz}, \mathrm{CDCl}_{3}\right)$ $\delta$ 21.3, 25.6, 52.7, 59.0, 65.2, 81.8, 125.7, 128.7, 137.4, 149.4. HRMS (EI) $m / z$ calcd for $\mathrm{C}_{20} \mathrm{H}_{30} \mathrm{BNO}_{3}: 343.2319$, found 343.2320 .<smiles></smiles>

4-(3-Phenyl-2-p-tolyl-allyl)morpholine: This compound was obtained as a mixture of isomers $(Z / E=$ 53/47). ${ }^{1} \mathrm{H}$ NMR (400 MHz, CDCl 3 ) $\delta 2.34$ (s, 3H, E-isomer), 2.35 (t, $J=4.4 \mathrm{~Hz}, 4 \mathrm{H}, \mathrm{Z}$-isomer), $2.36(\mathrm{~s}, 3 \mathrm{H}$, Z-isomer), $2.52(\mathrm{t}, J=4.4 \mathrm{~Hz}, 4 \mathrm{H}, E$-isomer), 3.27 (s, 2H, E-isomer), 3.51 (s, 2H, Z-isomer), 3.58 (t, $J=4.4$ $\mathrm{Hz}, 4 \mathrm{H}, \mathrm{Z}$-isomer), $3.70(\mathrm{t}, J=4.4 \mathrm{~Hz}, 4 \mathrm{H}, E$-isomer), 6.57 (s, 1H, E-isomer), 6.91 (s, 1H, Z-isomer), 6.95-6.98 (m, 2H), 7.05-7.11 (m, 7H), 7.14-7.16 (m, 2H), 7.22-7.26 (m, 1H), 7.32-7.36 (m, 2H), 7.40-7.45 (m, $4 \mathrm{H}) .{ }^{13} \mathrm{C}$ NMR (100 MHz, CDCl$) \delta 21.2$ (Z-isomer), 21.4 (E-isomer), 53.4 (Z-isomer), 53.7 (E-isomer), 57.1 (Z-isomer), 67.02 ( $E$-isomer), 67.04 (Z-isomer), 67.5 ( $E$-isomer), 126.36, 126.41, 126.7, 127.7, 128.0, 128.4, 128.7, 128.8, 128.98, 129.02, 129.2 (E-isomer), 131.9 (Z-isomer), 136.5, 136.7, 136.8, 137.3, 137.6, 137.9, 138.2, 140.0. HRMS (EI) $\mathrm{m} / z$ calcd for $\mathrm{C}_{20} \mathrm{H}_{23} \mathrm{NO}$ : 293.1780, found 293.1784.<smiles>[Z20]C1CN(C/C(=C\C2CCCCC2)c2ccc(C)cc2)CCO1</smiles>

4-(3-Cyclohexyl-2-p-tolyl-allyl)morpholine: This compound was obtained as a mixture of isomers (Z/E $=79 / 21) .{ }^{1} \mathrm{H}$ NMR $\left(400 \mathrm{MHz}, \mathrm{CDCl}_{3}\right) \delta 1.08-1.36(\mathrm{~m}, 12 \mathrm{H}), 1.58-1.77(\mathrm{~m}, 10 \mathrm{H}), 2.33(\mathrm{~s}, 3 \mathrm{H}, \mathrm{Z}$-isomer), 2.35 (s, 3H, E-isomer), $2.47(\mathrm{t}, J=4.4 \mathrm{~Hz}, 8 \mathrm{H}), 3.19(\mathrm{~s}, 2 \mathrm{H}, E$-isomer), $3.41(\mathrm{~s}, 2 \mathrm{H}, \mathrm{Z}$-isomer), $3.64(\mathrm{t}, J=4.4 \mathrm{~Hz}$, $4 \mathrm{H}, \mathrm{Z}$-isomer), 3.69 (t, $J=4.4 \mathrm{~Hz}, 4 \mathrm{H}, E$-isomer), $5.43(\mathrm{~d}, J=10.0 \mathrm{~Hz}, 1 \mathrm{H}, E$-isomer), $5.70(\mathrm{~d}, J=10.0 \mathrm{~Hz}$, $1 \mathrm{H}, \mathrm{Z}$-isomer), 7.08 (d, J = 8.0 Hz, 2H, Z-isomer), $7.13(\mathrm{~d}, J=8.0 \mathrm{~Hz}, 2 \mathrm{H}, E$-isomer), $7.35(\mathrm{~d}, J=8.0 \mathrm{~Hz}$, $4 \mathrm{H}) .{ }^{13} \mathrm{C}$ NMR (100 MHz, $\left.\mathrm{CDCl}_{3}\right) \delta 21.2$ (Z-isomer), 21.3 (E-isomer), 25.7 (E-isomer), 26.0 (Z-isomer), 26.07 ( $E$-isomer), 26.10 (Z-isomer), 33.2 (Z-isomer), 33.5 ( $E$-isomer), 37.5 ( $E$-isomer), 37.6 (Z-isomer), 53.2 (Z-isomer), 57.0 ( $(E$-isomer), 66.7 ( $E$-isomer), 66.9 (Z-isomer), 126.2, 128.0 ( $E$-isomer), 128.6 ( $Z$-isomer), 132.3 (E-isomer), 136.1 (Z-isomer), 139.1 (E-isomer), 139.9 (Z-isomer). HRMS (EI) $\mathrm{m} / \mathrm{z}$ calcd for $\mathrm{C}_{20} \mathrm{H}_{29} \mathrm{NO}: 299.2249$, found 299.2248. 
Typical Procedure for One-pot Four-component Assembly Reaction of Allenylboronate Pinacol Ester, Amines, and Two Aryl Iodides (Table 1).

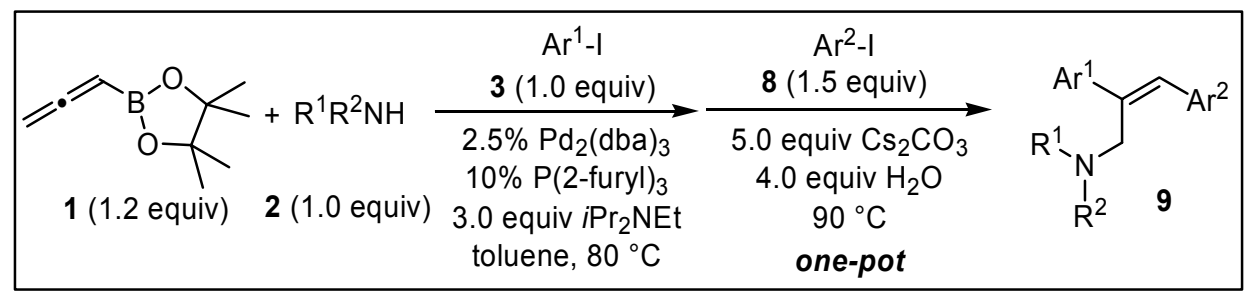

A mixture of $\mathrm{Pd}_{2}(\mathrm{dba})_{3}(6.9 \mathrm{mg}, 7.5 \mu \mathrm{mol}, 2.5 \mathrm{~mol} \%)$ and $\mathrm{P}(2 \text {-furyl })_{3}(7.0 \mathrm{mg}, 30 \mu \mathrm{mol}, 10 \mathrm{~mol} \%)$ in dry toluene $(0.5 \mathrm{~mL})$ was stirred at room temperature for 20 min under argon. To this mixture were added iodobenzene (3a; $60.2 \mathrm{mg}, 0.30 \mathrm{mmol}$ ), allenylboronate pinacol ester (1; $59.8 \mathrm{mg}, 0.36 \mathrm{mmol}$ ), morpholine (2b; $31.4 \mathrm{mg}, 0.36 \mathrm{mmol}), i-\operatorname{Pr} 2 \mathrm{NEt}(116.3 \mathrm{mg}, 0.90 \mathrm{mmol})$, and dry toluene $(1.0 \mathrm{~mL})$ and the resultant mixture was further stirred at $80{ }^{\circ} \mathrm{C}$ for $24 \mathrm{~h}$. To this mixture were added 4-iodoacetophenone (8b; $110.7 \mathrm{mg}, 0.45 \mathrm{mmol}), \mathrm{Cs}_{2} \mathrm{CO}_{3}(488.7 \mathrm{mg}, 1.5 \mathrm{mmol}), \mathrm{H}_{2} \mathrm{O}(21.6 \mathrm{mg}, 1.2 \mathrm{mmol})$, and dry toluene (0.5 $\mathrm{mL}$ ) at room temperature and the resultant mixture was further stirred at $90{ }^{\circ} \mathrm{C}$ for $24 \mathrm{~h}$. After cooling the reaction mixture to room temperature, catalyst and salts were removed by filtration through a short silica gel pad (EtOAc). The filtrate was evaporated and the residue was subjected to silica gel chromatography (hexane/EtOAc $=5 / 1$ to $3 / 1$ ) to afford 9 bab $(69.0 \mathrm{mg}, 73 \%)$ as colorless oil.<smiles>CC(=O)c1ccc(/C=C(\CN2CCOCC2)c2ccccc2)cc1</smiles>

9bab: 73\% yield from $\mathbf{1}$, morpholine (2b), iodobenzene (3a), and 4-iodoacetophenone (8b). ${ }^{1} \mathrm{H}$ NMR (400 $\left.\mathrm{MHz}, \mathrm{CDCl}_{3}\right) \delta 2.36(\mathrm{t}, J=4.4 \mathrm{~Hz}, 4 \mathrm{H}), 2.62(\mathrm{~s}, 3 \mathrm{H}), 3.52(\mathrm{~s}, 2 \mathrm{H}), 3.58(\mathrm{t}, J=4.4 \mathrm{~Hz}, 4 \mathrm{H}), 6.94(\mathrm{~s}, 1 \mathrm{H})$, 7.29-7.39 (m, 3H), $7.55(\mathrm{~d}, J=8.4 \mathrm{~Hz}, 4 \mathrm{H}), 7.96(\mathrm{~d}, J=8.4 \mathrm{~Hz}, 2 \mathrm{H}) .{ }^{13} \mathrm{C} \mathrm{NMR}\left(100 \mathrm{MHz}, \mathrm{CDCl}_{3}\right) \delta 26.6,53.4$, 57.2, 66.9, 126.5, 127.4, 128.1, 129.3, 131.5, 135.3, 140.5, 142.3, 142.5, 197.2. HRMS (EI) $\mathrm{m} / \mathrm{z}$ calcd for $\mathrm{C}_{21} \mathrm{H}_{23} \mathrm{NO}_{2}: 321.1729$, found 321.1727 .<smiles>COc1ccc(/C=C(\CN2CCOCC2)c2ccc(C)cc2)cc1</smiles>

9bcd: 74\% yield from 1, morpholine (2b), 4-iodotoluene (3c), and 4-iodoanisole (8d). ${ }^{1} \mathrm{H}$ NMR (400 MHz, $\left.\mathrm{CDCl}_{3}\right) \delta 2.36(\mathrm{~s}, 3 \mathrm{H}), 2.37(\mathrm{t}, J=4.8 \mathrm{~Hz}, 4 \mathrm{H}), 3.50(\mathrm{~s}, 2 \mathrm{H}), 3.59(\mathrm{t}, J=4.8 \mathrm{~Hz}, 4 \mathrm{H}), 3.82(\mathrm{~s}, 3 \mathrm{H}), 6.85(\mathrm{~s}, 1 \mathrm{H})$, $6.89(\mathrm{~d}, J=8.8 \mathrm{~Hz}, 2 \mathrm{H}), 7.14(\mathrm{~d}, J=8.0 \mathrm{~Hz}, 2 \mathrm{H}), 7.40(\mathrm{~d}, J=8.8 \mathrm{~Hz}, 2 \mathrm{H}), 7.42(\mathrm{~d}, J=8.0 \mathrm{~Hz}, 2 \mathrm{H}) .{ }^{13} \mathrm{C} \mathrm{NMR}$ $\left(100 \mathrm{MHz}, \mathrm{CDCl}_{3}\right) \delta 21.6,53.8,55.6,57.6,67.4,113.8,126.7,129.1,130.7,130.9,132.1,136.8,137.2,140.8$, 158.7. HRMS (EI) $\mathrm{m} / z$ calcd for $\mathrm{C}_{21} \mathrm{H}_{25} \mathrm{NO}_{2}$ : 323.1885, found 323.1885 . 
<smiles>Cc1ccc(C=C(CN2CCOCC2)c2ccsc2)cc1</smiles>

9bec: $90 \%$ yield from 1, morpholine (2b), 3-iodothiophene (3e), and 4-iodotoluene (8c). ${ }^{1} \mathrm{H}$ NMR (400 $\left.\mathrm{MHz}, \mathrm{CDCl}_{3}\right) \delta 2.36(\mathrm{~s}, 3 \mathrm{H}), 2.39(\mathrm{t}, J=4.4 \mathrm{~Hz}, 4 \mathrm{H}), 3.45(\mathrm{~s}, 2 \mathrm{H}), 3.63(\mathrm{t}, J=4.4 \mathrm{~Hz}, 4 \mathrm{H}), 7.08(\mathrm{~s}, 1 \mathrm{H}), 7.15$ $(\mathrm{d}, J=8.0 \mathrm{~Hz}, 2 \mathrm{H}), 7.26(\mathrm{dd}, J=5.2,2.8 \mathrm{~Hz}, 1 \mathrm{H}), 7.29(\mathrm{~d}, J=8.0 \mathrm{~Hz}, 2 \mathrm{H}), 7.36(\mathrm{dd}, J=5.2,1.2 \mathrm{~Hz}, 1 \mathrm{H}), 7.61$ $(\mathrm{dd}, J=2.8,1.2 \mathrm{~Hz}, 1 \mathrm{H}) .{ }^{13} \mathrm{C} \mathrm{NMR}\left(100 \mathrm{MHz}, \mathrm{CDCl}_{3}\right) \delta$ 21.3, 53.3, 57.4, 67.1, 121.0, 124.7, 126.1, 128.7, 129.1, 130.9, 132.1, 134.2, 136.5, 143.6. HRMS (EI) m/z calcd for $\mathrm{C}_{18} \mathrm{H}_{21} \mathrm{NOS}: 299.1344$, found 299.1344.<smiles>FC(F)(F)c1ccc(C=Cc2ccccc2)cc1</smiles>

9bfa: $80 \%$ yield from 1 , morpholine (2b), 4-iodobenzotrifluoride (3f), and iodobenzene (8a). ${ }^{1} \mathrm{H}$ NMR $\left(400 \mathrm{MHz}, \mathrm{CDCl}_{3}\right) \delta 2.34(\mathrm{t}, J=4.4 \mathrm{~Hz}, 4 \mathrm{H}), 3.54(\mathrm{~s}, 2 \mathrm{H}), 3.56(\mathrm{t}, J=4.4 \mathrm{~Hz}, 4 \mathrm{H}), 7.00(\mathrm{~s}, 1 \mathrm{H}), 7.27-7.31(\mathrm{~m}$, $1 \mathrm{H}), 7.35-7.42(\mathrm{~m}, 4 \mathrm{H}), 7.59(\mathrm{~d}, J=8.4 \mathrm{~Hz}, 2 \mathrm{H}), 7.67(\mathrm{~d}, J=8.4 \mathrm{~Hz}, 2 \mathrm{H}) .{ }^{13} \mathrm{C}$ NMR $\left(100 \mathrm{MHz}, \mathrm{CDCl}_{3}\right) \delta 53.3$, $56.9,66.9,124.2\left(\mathrm{q}, J_{\mathrm{C}-\mathrm{F}}=269.0 \mathrm{~Hz}\right), 124.9\left(\mathrm{q}, J_{\mathrm{C}-\mathrm{F}}=3.6 \mathrm{~Hz}\right), 126.9,127.2,128.1,129.0\left(\mathrm{q}, J_{\mathrm{C}-\mathrm{F}}=32.1 \mathrm{~Hz}\right)$, 129.1, 134.0, 136.9, 137.4, 146.2 (q, Jc-F $=1.2 \mathrm{~Hz}$ ). HRMS (EI) $\mathrm{m} / \mathrm{z}$ calcd for $\mathrm{C}_{20} \mathrm{H}_{20} \mathrm{~F}_{3} \mathrm{NO}$ : 347.1497, found 347.1499 .<smiles>COc1cccc(C=C(c2cccc3ccccc23)C2CCCCC2)c1</smiles>

9bgh: $59 \%$ yield from 1, morpholine (2b), 1-iodonaphthalene (3g), and 3-iodoanisole (8h). ${ }^{1} \mathrm{H}$ NMR (400 $\left.\mathrm{MHz}, \mathrm{CDCl}_{3}\right) \delta 2.37(\mathrm{t}, J=4.4 \mathrm{~Hz}, 4 \mathrm{H}), 3.54(\mathrm{t}, J=4.4 \mathrm{~Hz}, 4 \mathrm{H}), 3.59(\mathrm{~s}, 2 \mathrm{H}), 3.84(\mathrm{~s}, 3 \mathrm{H}), 6.77(\mathrm{~s}, 1 \mathrm{H}), 6.85$ $(\mathrm{dd}, J=8.4,2.4 \mathrm{~Hz}, 1 \mathrm{H}), 7.09(\mathrm{dd}, J=7.6,0.8 \mathrm{~Hz}, 1 \mathrm{H}), 7.22-7.32(\mathrm{~m}, 2 \mathrm{H}), 7.41-7.48(\mathrm{~m}, 4 \mathrm{H}), 7.76-7.78(\mathrm{~m}$, 1H), 7.84-7.86 (m, 1H), 8.05-8.08 (m, 1H). ${ }^{13} \mathrm{C}$ NMR (100 MHz, CDCl $) \delta$ 53.7, 55.3, 60.1, 67.0, 112.8, 114.7, 121.9, 125.0, 125.39, 125.44, 125.5, 125.7, 127.1, 128.4, 129.0, 131.3, 133.8, 135.5, 138.2, 138.5, 142.1, 159.3. HRMS (EI) $m / z$ calcd for $\mathrm{C}_{24} \mathrm{H}_{25} \mathrm{NO}_{2}: 359.1885$, found 359.1882 .<smiles>Cc1ccc(C=Cc2ccccc2)cc1</smiles>

9cca: 66\% yield from 1, pyrrolidine (2c), 4-iodotoluene (3c), and iodobenzene (8a). ${ }^{1} \mathrm{H}$ NMR (400 MHz, $\left.\mathrm{CDCl}_{3}\right) \delta 1.66(\mathrm{ddd}, J=13.2,6.8,6.8 \mathrm{~Hz}, 4 \mathrm{H}), 2.36(\mathrm{~s}, 3 \mathrm{H}), 2.41-2.45(\mathrm{~m}, 4 \mathrm{H}), 3.67(\mathrm{~s}, 2 \mathrm{H}), 6.82(\mathrm{~s}, 1 \mathrm{H}), 7.16$ $(\mathrm{d}, J=8.0 \mathrm{~Hz}, 2 \mathrm{H}), 7.23-7.27(\mathrm{~m}, 1 \mathrm{H}), 7.33-7.37(\mathrm{~m}, 2 \mathrm{H}), 7.42-7.45(\mathrm{~m}, 4 \mathrm{H}) .{ }^{13} \mathrm{C}$ NMR $\left(100 \mathrm{MHz}, \mathrm{CDCl}_{3}\right) \delta$ 21.3, 23.6, 54.0, 54.2, 126.5, 126.6, 128.0, 128.8, 129.2, 131.2, 136.7, 137.7, 139.6, 140.1. HRMS (EI) $\mathrm{m} / \mathrm{z}$ calcd for $\mathrm{C}_{20} \mathrm{H}_{23} \mathrm{~N}$ : 277.1830, found 277.1833. 
<smiles>COc1ccc(/C=C2\CCCN(CC(C)(C)C)C2)cc1</smiles>

9cde: 69\% yield from 1, pyrrolidine (2c), 4-iodoanisole (3d), and 3-iodothiophene (8e). ${ }^{1} \mathrm{H}$ NMR (400 $\left.\mathrm{MHz} \mathrm{CDCl}_{3}\right) \delta 1.67(\mathrm{ddd}, J=13.2,6.8,6.4 \mathrm{~Hz}, 4 \mathrm{H}), 2.43(\mathrm{ddd}, J=13.2,6.8,6.4 \mathrm{~Hz}, 4 \mathrm{H}), 3.64(\mathrm{~s}, 2 \mathrm{H}), 3.81$ $(\mathrm{s}, 3 \mathrm{H}), 6.70(\mathrm{~s}, 1 \mathrm{H}), 6.87(\mathrm{~d}, J=8.8 \mathrm{~Hz}, 2 \mathrm{H}), 7.19(\mathrm{dd}, J=4.8,1.2 \mathrm{~Hz}, 1 \mathrm{H}), 7.27(\mathrm{dd}, J=4.8,3.2 \mathrm{~Hz}, 1 \mathrm{H})$, $7.44(\mathrm{~d}, J=8.8 \mathrm{~Hz}, 2 \mathrm{H}), 7.47(\mathrm{dd}, J=3.2,1.2 \mathrm{~Hz}, 1 \mathrm{H}) .{ }^{13} \mathrm{C} \mathrm{NMR}\left(100 \mathrm{MHz}, \mathrm{CDCl}_{3}\right) \delta 23.6,54.1,55.1,55.3$, 113.5, 123.6, 124.5, 124.6, 127.6, 129.3, 136.0, 138.8, 139.0, 158.6. HRMS (EI) $m / z$ calcd for $\mathrm{C}_{18} \mathrm{H}_{21} \mathrm{NOS}$ : 299.1344, found 299.1345 .<smiles>CCOC(=O)c1ccc(C=Cc2ccccc2C)cc1</smiles>

9cij: 66\% yield from 1, pyrrolidine (2c), ethyl 4-iodobenzoate (3i), and 2-iodotoluene (8j). ${ }^{1} \mathrm{H}$ NMR (400 $\left.\mathrm{MHz} \mathrm{CDCl}_{3}\right) \delta 1.39(\mathrm{t}, J=6.8 \mathrm{~Hz}, 3 \mathrm{H}), 1.59(\mathrm{ddd}, J=13.6,6.8,6.8 \mathrm{~Hz}, 4 \mathrm{H}), 2.28(\mathrm{~s}, 3 \mathrm{H}), 2.32(\mathrm{ddd}, J=13.6$, $6.8,6.8 \mathrm{~Hz}, 4 \mathrm{H}), 3.55(\mathrm{~s}, 2 \mathrm{H}), 4.37(\mathrm{q}, J=6.8 \mathrm{~Hz}, 2 \mathrm{H}), 6.90(\mathrm{~s}, 1 \mathrm{H}), 7.18-7.20(\mathrm{~m}, 3 \mathrm{H}), 7.29-7.31(\mathrm{~m}, 1 \mathrm{H})$, $7.66(\mathrm{~d}, J=8.8 \mathrm{~Hz}, 2 \mathrm{H}), 8.02(\mathrm{~d}, J=8.8 \mathrm{~Hz}, 2 \mathrm{H}) .{ }^{13} \mathrm{C} \mathrm{NMR}\left(100 \mathrm{MHz}, \mathrm{CDCl}_{3}\right) \delta 14.5,20.2,23.6,53.8,54.1$, $60.8,125.3,126.6,127.2,128.9,129.3,129.4,129.6,131.4,136.4,136.5,139.4,147.0,166.3$. HRMS (EI) $\mathrm{m} / \mathrm{z}$ calcd for $\mathrm{C}_{23} \mathrm{H}_{27} \mathrm{NO}_{2}$ : 349.2042 , found 349.2038 .<smiles>COc1ccc(/C=C(\CN(C)Cc2ccccc2)c2ccc(C)cc2)cc1</smiles>

9dcd: $52 \%$ yield from $1, N$-benzylmethylamine (2d), 4-iodotoluene (3c), and 4-iodoanisole (8d). ${ }^{1} \mathrm{H}$ NMR $\left(400 \mathrm{MHz}, \mathrm{CDCl}_{3}\right) 2.06(\mathrm{~s}, 3 \mathrm{H}), 2.37(\mathrm{~s}, 3 \mathrm{H}), 3.41(\mathrm{~s}, 2 \mathrm{H}), 3.57(\mathrm{~s}, 2 \mathrm{H}), 3.83(\mathrm{~s}, 3 \mathrm{H}), 6.83(\mathrm{~s}, 1 \mathrm{H}), 6.88(\mathrm{~d}, J=$ $8.8 \mathrm{~Hz}, 2 \mathrm{H}), 7.12-7.25(\mathrm{~m}, 7 \mathrm{H}), 7.35-7.38(\mathrm{~m}, 4 \mathrm{H}) .{ }^{13} \mathrm{C} \mathrm{NMR}\left(100 \mathrm{MHz}, \mathrm{CDCl}_{3}\right) \delta 21.3,42.1,55.3,56.0,61.9$, $113.5,126.6,127.9,128.6,129.0,130.3,130.5,130.9,136.4,138.6,139.3,140.0,158.2$. HRMS (EI) $\mathrm{m} / \mathrm{z}$ calcd for $\mathrm{C}_{25} \mathrm{H}_{27} \mathrm{NO}$ : 357.2093, found 357.2092.<smiles>O=C(O)CN(Cc1ccccc1)C(=Cc1cccnc1)c1ccccc1</smiles>

9dak: $67 \%$ yield from $\mathbf{1}, \mathrm{N}$-benzylmethylamine (2d), iodobenzene (3a), and 3-iodopyridine $(8 \mathbf{k}) .{ }^{1} \mathrm{H}$ NMR (400 MHz, $\left.\mathrm{CDCl}_{3}\right) \delta 2.06(\mathrm{~s}, 3 \mathrm{H}), 3.40(\mathrm{~s}, 2 \mathrm{H}), 3.57(\mathrm{~s}, 2 \mathrm{H}), 6.83(\mathrm{~s}, 1 \mathrm{H}), 7.08-7.10(\mathrm{~m}, 2 \mathrm{H})$, 7.19-7.39 $(\mathrm{m}, 7 \mathrm{H}), 7.48-7.50(\mathrm{~m}, 2 \mathrm{H}), 7.75(\mathrm{dt}, J=7.6,1.6 \mathrm{~Hz}, 1 \mathrm{H}), 8.50(\mathrm{dd}, J=4.4,0.8 \mathrm{~Hz}, 1 \mathrm{H}), 8.66(\mathrm{~s}, 1 \mathrm{H}) .{ }^{13} \mathrm{C}$ NMR $\left(100 \mathrm{MHz}, \mathrm{CDCl}_{3}\right) \delta 42.0,56.0,61.8,122.9,126.8,126.9,127.4,127.8,127.9,128.0,128.9,133.2,136.2$, $138.9,142.0,142.6,147.6,150.2$. HRMS (EI) $\mathrm{m} / z$ calcd for $\mathrm{C}_{22} \mathrm{H}_{22} \mathrm{~N}_{2}: 314.1783$, found 314.1784. 
<smiles>CCOC(=O)c1ccc(/C=C(\CN(C)Cc2ccccc2)c2ccsc2)cc1</smiles>

9dei: $82 \%$ yield from $1, N$-benzylmethylamine (2d), 3-iodothiophene (3e), and ethyl 4-iodobenzoate (8i). ${ }^{1} \mathrm{H}$ NMR $\left(400 \mathrm{MHz}, \mathrm{CDCl}_{3}\right) \delta 1.41(\mathrm{t}, J=6.8 \mathrm{~Hz}, 3 \mathrm{H}), 2.08(\mathrm{~s}, 3 \mathrm{H}), 3.41(\mathrm{~s}, 2 \mathrm{H}), 3.52(\mathrm{~s}, 2 \mathrm{H}), 4.39(\mathrm{q}, J=6.8$ $\mathrm{Hz}, 2 \mathrm{H}), 7.09(\mathrm{~s}, 1 \mathrm{H}), 7.16-7.29(\mathrm{~m}, 6 \mathrm{H}), 7.33(\mathrm{dd}, J=5.2,1.2 \mathrm{~Hz}, 1 \mathrm{H}), 7.46(\mathrm{~d}, J=8.4 \mathrm{~Hz}, 2 \mathrm{H}), 7.57(\mathrm{dd}, J=$ 2.8, $1.2 \mathrm{~Hz}, 1 \mathrm{H}), 8.03(\mathrm{~d}, J=8.4 \mathrm{~Hz}, 2 \mathrm{H}) .{ }^{13} \mathrm{C} \mathrm{NMR}\left(100 \mathrm{MHz}, \mathrm{CDCl}_{3}\right) \delta 14.5,41.9,56.5,60.9,61.9,122.0$, $124.8,126.2,126.8,128.0,128.6,128.9,129.1,129.3,135.9,139.0,142.0,142.9,166.2$. HRMS (EI) $\mathrm{m} / \mathrm{z}$ calcd for $\mathrm{C}_{24} \mathrm{H}_{25} \mathrm{NO}_{2} \mathrm{~S}: 391.1606$, found 391.1608 .<smiles>CN(CC(=Cc1ccc(Cl)cc1)c1ccc(C#N)cc1)Cc1ccccc1</smiles>

9dlm: 74\% yield from $N$-benzylmethylamine (2d), 3-iodobenzonitrile (31), and 4-chloroiodobenzene (8m). ${ }^{1} \mathrm{H}$ NMR (400 MHz, CDCl 3 ) $\delta 2.04(\mathrm{~s}, 3 \mathrm{H}), 3.36(\mathrm{~s}, 2 \mathrm{H}), 3.55(\mathrm{~s}, 2 \mathrm{H}), 6.90(\mathrm{~s}, 1 \mathrm{H}), 7.05$ (dd, J = 7.6, 2.4 $\mathrm{Hz}, 2 \mathrm{H}), 7.20-7.26(\mathrm{~m}, 3 \mathrm{H}), 7.29(\mathrm{~d}, J=8.4 \mathrm{~Hz}, 2 \mathrm{H}), 7.34(\mathrm{~d}, J=8.4 \mathrm{~Hz}, 2 \mathrm{H}), 7.53(\mathrm{~d}, J=8.4 \mathrm{~Hz}, 2 \mathrm{H}), 7.61(\mathrm{~d}$, $J=8.4 \mathrm{~Hz}, 2 \mathrm{H}) .{ }^{13} \mathrm{C} \mathrm{NMR}\left(100 \mathrm{MHz}, \mathrm{CDCl}_{3}\right) \delta 42.0,55.2,61.8,110.6,118.9,127.0,127.5,128.0,128.3,128.8$, 130.4, 131.6, 132.4, 133.1, 135.0, 138.5, 139.5, 146.6. HRMS (EI) $\mathrm{m} / \mathrm{z}$ calcd for $\mathrm{C}_{24} \mathrm{H}_{21} \mathrm{ClN}_{2}: 372.1393$, found 372.1394 .

\section{Synthesis of Various Allylic Amines through C-B Functionalization of 4 (Scheme 1).}

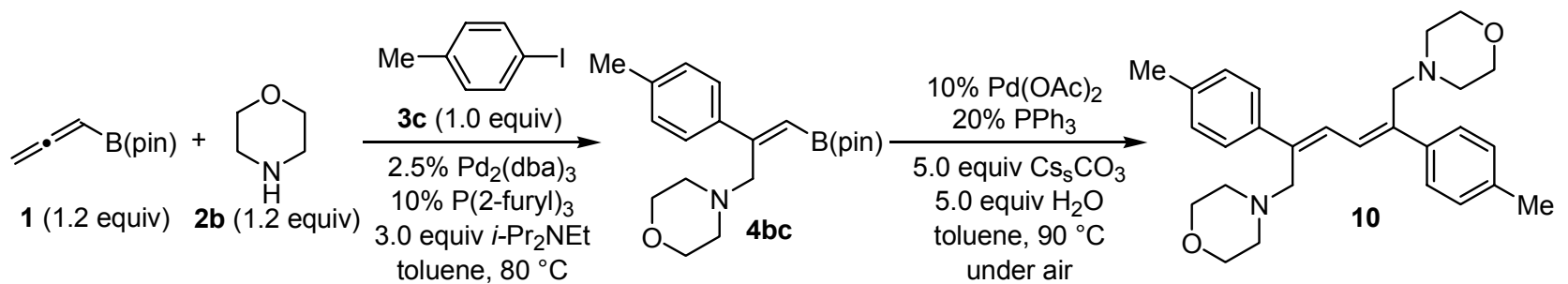

A mixture of $\mathrm{Pd}_{2}(\mathrm{dba}) 3(6.9 \mathrm{mg}, 7.5 \mu \mathrm{mol}, 2.5 \mathrm{~mol} \%)$ and $\mathrm{P}(2$-furyl $) 3(7.0 \mathrm{mg}, 30 \mu \mathrm{mol}, 10 \mathrm{~mol} \%)$ in dry toluene $(0.5 \mathrm{~mL})$ was stirred at room temperature for $20 \mathrm{~min}$ under argon. To this mixture were added 4-iodotoluene (3c; $64.9 \mathrm{mg}, 0.30 \mathrm{mmol})$, allenylboronate pinacol ester (1; $59.8 \mathrm{mg}, 0.36 \mathrm{mmol})$, morpholine $(\mathbf{2} \mathbf{b} ; 31.4 \mathrm{mg}, 0.36 \mathrm{mmol}), i-\operatorname{Pr} 2 \mathrm{NEt}(116.3 \mathrm{mg}, 0.90 \mathrm{mmol})$, and dry toluene $(1.0 \mathrm{~mL})$ and the resultant mixture was further stirred at $80{ }^{\circ} \mathrm{C}$ for $24 \mathrm{~h}$. After cooling the reaction mixture to room temperature, catalyst and salts were removed by filtration (toluene). The filtrate was evaporated to give a crude $4 \mathrm{bc}$, which was directly used for the next operation.

A mixture of $4 \mathrm{bc}, \mathrm{Pd}(\mathrm{OAc}) 2(6.7 \mathrm{mg}, 30 \mu \mathrm{mol}), \mathrm{PPh}_{3}(15.7 \mathrm{mg}, 60 \mu \mathrm{mol}), \mathrm{Cs}_{2} \mathrm{CO}_{3}(489 \mathrm{mg}, 1.5 \mathrm{mmol})$, and $\mathrm{H}_{2} \mathrm{O}(27 \mathrm{mg}, 1.5 \mathrm{mmol})$ in toluene $(2.0 \mathrm{~mL})$ was stirred at $90{ }^{\circ} \mathrm{C}$ for $12 \mathrm{~h}$ under an air atmosphere. After cooling the reaction mixture to room temperature, catalyst and salts were removed by filtration through a short silica gel pad (EtOAc). The residue was subjected to silica gel chromatography (hexane/EtOAc $=5 / 1$ to $2 / 1)$ to afford $10\left(40.7 \mathrm{mg}, 63 \%\right.$ in 2 steps) as pale yellow solid: ${ }^{1} \mathrm{H}$ NMR (400 $\left.\mathrm{MHz}_{\mathrm{CDCl}}\right) \delta 2.37(\mathrm{~s}, 6 \mathrm{H}), 2.50(\mathrm{t}, J=4.0 \mathrm{~Hz}, 8 \mathrm{H}), 3.57(\mathrm{~s}, 4 \mathrm{H}), 3.65(\mathrm{t}, J=4.0 \mathrm{~Hz}, 8 \mathrm{H}), 7.13(\mathrm{~s}, 2 \mathrm{H}), 7.16$ $(\mathrm{d}, J=8.0 \mathrm{~Hz}, 4 \mathrm{H}), 7.49(\mathrm{~d}, J=8.0 \mathrm{~Hz}, 4 \mathrm{H}) .{ }^{13} \mathrm{C} \mathrm{NMR}\left(100 \mathrm{MHz}, \mathrm{CDCl}_{3}\right) \delta 21.3,53.5,56.9,67.1,126.1,126.6$, 128.9, 137.0, 139.6. HRMS (EI) $\mathrm{m} / z$ calcd for $\mathrm{C}_{28} \mathrm{H}_{36} \mathrm{~N}_{2} \mathrm{O}_{2}$ : 432.2777, found 432.2781. 


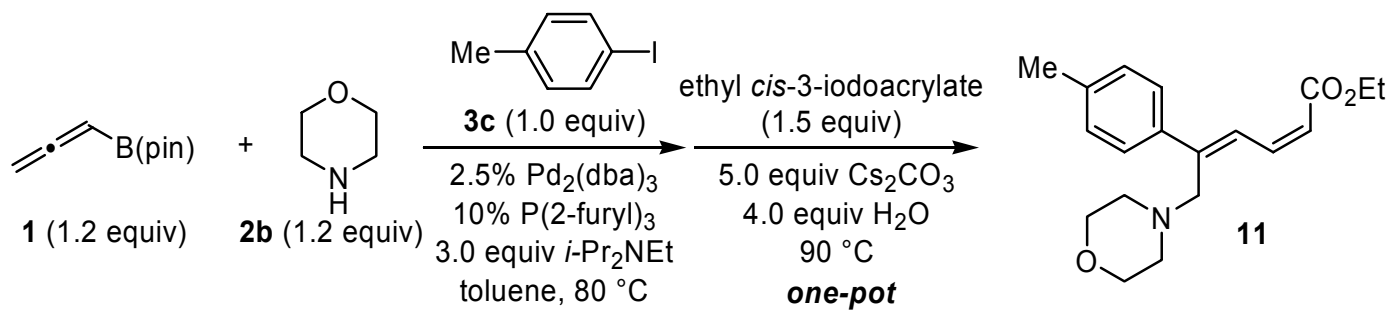

A mixture of $\mathrm{Pd}_{2}(\mathrm{dba})_{3}(6.9 \mathrm{mg}, 7.5 \mu \mathrm{mol}, 2.5 \mathrm{~mol} \%)$ and $\mathrm{P}(2$-furyl $) 3(7.0 \mathrm{mg}, 30 \mu \mathrm{mol}, 10 \mathrm{~mol} \%)$ in dry toluene $(0.5 \mathrm{~mL})$ was stirred at room temperature for $20 \mathrm{~min}$ under argon. To this mixture were added 4-iodotoluene (3c; $63.0 \mathrm{mg}, 0.29 \mathrm{mmol})$, allenylboronate pinacol ester $(\mathbf{1} ; 59.8 \mathrm{mg}, 0.36 \mathrm{mmol})$, morpholine $(\mathbf{2 b} ; 31.4 \mathrm{mg}, 0.36 \mathrm{mmol}), i-\operatorname{Pr} 2 \mathrm{NEt}(116.3 \mathrm{mg}, 0.90 \mathrm{mmol})$, and dry toluene $(1.0 \mathrm{~mL})$ and the resultant mixture was further stirred at $80{ }^{\circ} \mathrm{C}$ for $24 \mathrm{~h}$. To this mixture were added ethyl cis-3-iodoacrylate (101.7 mg, $0.45 \mathrm{mmol}), \mathrm{Cs}_{2} \mathrm{CO}_{3}(488.7 \mathrm{mg}, 1.5 \mathrm{mmol}), \mathrm{H}_{2} \mathrm{O}(21.6 \mathrm{mg}, 1.2 \mathrm{mmol})$, and dry toluene $(0.5 \mathrm{~mL})$ at room temperature and the resultant mixture was further stirred at $90{ }^{\circ} \mathrm{C}$ for $24 \mathrm{~h}$. After cooling the reaction mixture to room temperature, catalyst and salts were removed by filtration through a short silica gel pad (EtOAc). The filtrate was evaporated and the residue was subjected to silica gel chromatography (hexane/EtOAc $=6 / 1$ to $4 / 1)$ to afford $11(65.9 \mathrm{mg}, 72 \%)$ as colorless oil: ${ }^{1} \mathrm{H}$ $\operatorname{NMR}\left(400 \mathrm{MHz}, \mathrm{CDCl}_{3}\right) \delta 1.32(\mathrm{t}, J=7.2 \mathrm{~Hz}, 3 \mathrm{H}), 2.35(\mathrm{~s}, 3 \mathrm{H}), 2.46(\mathrm{t}, J=4.4 \mathrm{~Hz}, 4 \mathrm{H}), 3.57(\mathrm{~s}, 2 \mathrm{H}), 3.63(\mathrm{t}$, $J=4.4 \mathrm{~Hz}, 4 \mathrm{H}), 4.23(\mathrm{q}, J=7.2 \mathrm{~Hz}, 2 \mathrm{H}), 5.97(\mathrm{~d}, J=14.8 \mathrm{~Hz}, 1 \mathrm{H}), 6.65(\mathrm{~d}, J=12.0 \mathrm{~Hz}, 1 \mathrm{H}), 7.14(\mathrm{~d}, J=8.4$ $\mathrm{Hz}, 2 \mathrm{H}), 7.47(\mathrm{~d}, J=8.4 \mathrm{~Hz}, 2 \mathrm{H}), 7.88(\mathrm{dd}, J=14.8,12.0 \mathrm{~Hz}, 1 \mathrm{H}) .{ }^{13} \mathrm{C}$ NMR $\left(100 \mathrm{MHz}, \mathrm{CDCl}_{3}\right) \delta 14.4,21.3$, 53.4, 57.1, 60.3, 67.0, 122.3, 126.4, 127.7, 128.9, 138.1, 139.8, 144.3, 166.9. HRMS (EI) $\mathrm{m} / \mathrm{z}$ calcd for $\mathrm{C}_{19} \mathrm{H}_{25} \mathrm{NO}_{3}: 315.1834$, found 315.1835.

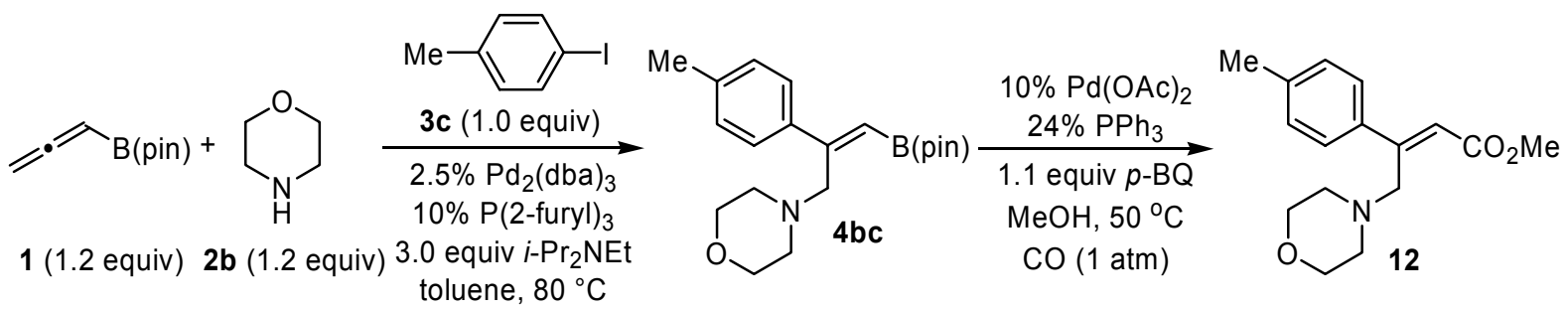

A mixture of $\mathrm{Pd}_{2}(\mathrm{dba})_{3}(6.9 \mathrm{mg}, 7.5 \mu \mathrm{mol}, 2.5 \mathrm{~mol} \%)$ and $\mathrm{P}(2 \text {-furyl })_{3}(7.0 \mathrm{mg}, 30 \mu \mathrm{mol}, 10 \mathrm{~mol} \%)$ in dry toluene $(0.5 \mathrm{~mL})$ was stirred at room temperature for 20 min under argon. To this mixture were added 4-iodotoluene (3c; $64.6 \mathrm{mg}, 0.30 \mathrm{mmol})$, allenylboronate pinacol ester $(\mathbf{1} ; 59.8 \mathrm{mg}, 0.36 \mathrm{mmol})$, morpholine $(\mathbf{2} \mathbf{b} ; 31.4 \mathrm{mg}, 0.36 \mathrm{mmol}), i-\operatorname{Pr} 2 \mathrm{NEt}(116.3 \mathrm{mg}, 0.90 \mathrm{mmol})$, and dry toluene $(1.0 \mathrm{~mL})$ and the resultant mixture was further stirred at $80{ }^{\circ} \mathrm{C}$ for $24 \mathrm{~h}$. After cooling the reaction mixture to room temperature, catalyst and salts were removed by filtration (toluene). The filtrate was evaporated to give a crude $\mathbf{4 b c}$, which was directly used for the next operation.

A dry schlenk tube was charged with $\mathrm{Pd}(\mathrm{OAc}) 2(6.7 \mathrm{mg}, 30 \mu \mathrm{mol}, 10 \mathrm{~mol} \%), \mathrm{PPh}_{3}(19.2 \mathrm{mg}, 73 \mu \mathrm{mol}$, $24 \mathrm{~mol} \%$ ), and $p$-benzoquinone ( $35.7 \mathrm{mg}, 0.33 \mathrm{mmol}$ ). Then the tube was flushed with carbon monoxide. To this mixture was added a solution of $4 \mathbf{b c}$ in anhydrous $\mathrm{MeOH}(3 \mathrm{~mL})$, and then the solution was stirred at $50{ }^{\circ} \mathrm{C}$ for $10 \mathrm{~h}$ under atmospheric pressure of carbon monoxide. The reaction mixture was filtered, and the filtrate was evaporated. Water was added to the residue, and the aqueous phase was extracted with EtOAc. The combined organic phase was washed with brine and dried over $\mathrm{Na}_{2} \mathrm{SO}_{4}$. The filtrate was evaporated, and the residue was subjected to silica gel chromatography (hexane/EtOAc $=$ $5 / 1$ to $4 / 1)$ to afford 12 (50.0 mg, 61\% in 2 steps) as colorless oil. $\left.{ }^{1} \mathrm{H} \mathrm{NMR} \mathrm{(400} \mathrm{MHz,} \mathrm{CDCl}_{3}\right) \delta 2.37(\mathrm{~s}, 3 \mathrm{H})$, $2.50(\mathrm{t}, J=4.4 \mathrm{~Hz}, 4 \mathrm{H}), 3.60(\mathrm{t}, J=4.4 \mathrm{~Hz}, 4 \mathrm{H}), 3.75(\mathrm{~s}, 3 \mathrm{H}), 3.96(\mathrm{~s}, 2 \mathrm{H}), 6.20(\mathrm{~s}, 1 \mathrm{H}), 7.16(\mathrm{~d}, J=8.0 \mathrm{~Hz}$, $2 \mathrm{H}), 7.44(\mathrm{~d}, J=8.0 \mathrm{~Hz}, 2 \mathrm{H}) .{ }^{13} \mathrm{C} \mathrm{NMR}\left(100 \mathrm{MHz}, \mathrm{CDCl}_{3}\right) \delta 21.4,51.3,53.3,55.9,66.9,119.8,126.8,128.9$, 137.4, 139.0, 153.7, 166.6. HRMS (EI) $m / z$ calcd for $\mathrm{C}_{16} \mathrm{H}_{21} \mathrm{NO}_{3}$ : 275.1521, found 275.1525. 


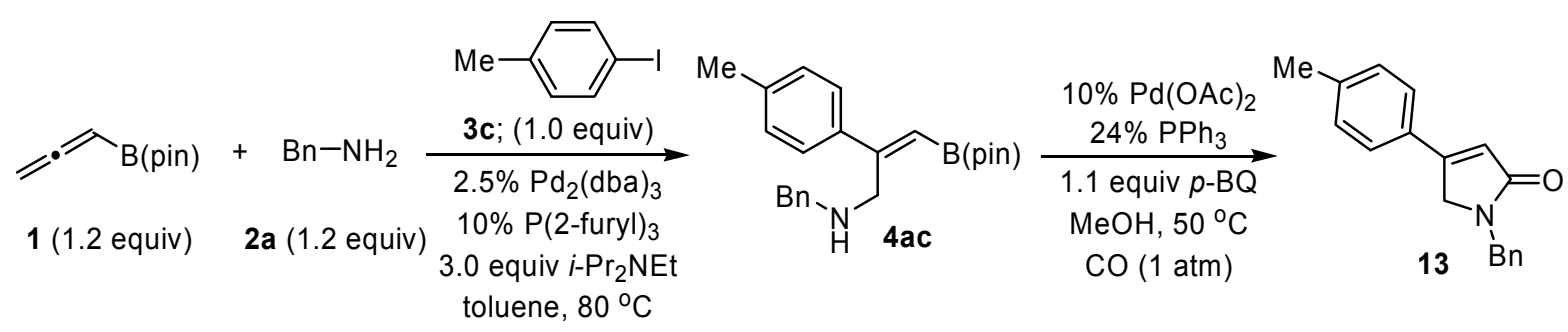

A mixture of $\mathrm{Pd}_{2}(\mathrm{dba})_{3}(6.9 \mathrm{mg}, 7.5 \mu \mathrm{mol}, 2.5 \mathrm{~mol} \%)$ and $\mathrm{P}(2$-furyl $) 3(7.0 \mathrm{mg}, 30 \mu \mathrm{mol}, 10 \mathrm{~mol} \%)$ in dry toluene $(0.5 \mathrm{~mL})$ was stirred at room temperature for $20 \mathrm{~min}$ under argon. To this mixture were added 4-iodotoluene (3c; $64.2 \mathrm{mg}, 0.29 \mathrm{mmol})$, allenylboronate pinacol ester (1; $59.8 \mathrm{mg}, 0.36 \mathrm{mmol})$, benzylamine (2a; $38.6 \mathrm{mg}, 0.36 \mathrm{mmol}), i-\operatorname{Pr} 2 \mathrm{NEt}(116.3 \mathrm{mg}, 0.90 \mathrm{mmol})$, and dry toluene $(1.0 \mathrm{~mL})$ and the resultant mixture was further stirred at $80{ }^{\circ} \mathrm{C}$ for $24 \mathrm{~h}$. After cooling the reaction mixture to room temperature, catalyst and salts were removed by filtration (toluene). The filtrate was evaporated to give a crude 4 ac, which was directly used for the next operation.

A dry schlenk tube was charged with $\mathrm{Pd}(\mathrm{OAc}) 2(6.7 \mathrm{mg}, 30 \mu \mathrm{mol}, 10 \mathrm{~mol} \%), \mathrm{PPh}_{3}(19.2 \mathrm{mg}, 73 \mu \mathrm{mol}$, $24 \mathrm{~mol} \%$ ), and $p$-benzoquinone ( $35.7 \mathrm{mg}, 0.33 \mathrm{mmol})$. Then the tube was flushed with carbon monoxide. To this mixture was added a solution of $4 \mathrm{ac}$ in anhydrous $\mathrm{MeOH}(3 \mathrm{~mL})$, and then the solution was stirred at $50{ }^{\circ} \mathrm{C}$ for $10 \mathrm{~h}$ under atmospheric pressure of carbon monoxide. The reaction mixture was filtered, and the filtrate was evaporated. Water was added to the residue, and the aqueous phase was extracted with EtOAc. The combined organic phase was washed with brine and dried over $\mathrm{Na}_{2} \mathrm{SO}_{4}$. The filtrate was evaporated, and the residue was subjected to silica gel chromatography (hexane/EtOAc $=$ 2/1 to 1/1) to afford 13 (47.7 mg, 62\% in 2 steps) as colorless solid: $\left.{ }^{1} \mathrm{H} \mathrm{NMR} \mathrm{(400} \mathrm{MHz,} \mathrm{CDCl} 3\right) \delta 2.35$ (s, $3 \mathrm{H}), 4.19(\mathrm{~d}, J=1.6 \mathrm{~Hz}, 2 \mathrm{H}), 4.68(\mathrm{~s}, 2 \mathrm{H}), 6.41(\mathrm{t}, J=1.6 \mathrm{~Hz}, 1 \mathrm{H}), 7.16(\mathrm{~d}, J=8.4 \mathrm{~Hz}, 2 \mathrm{H}), 7.24-7.35(\mathrm{~m}, 7 \mathrm{H})$. ${ }^{13} \mathrm{C}$ NMR $\left(100 \mathrm{MHz}, \mathrm{CDCl}_{3}\right) \delta 21.5,46.0,51.8,119.1,125.5,127.4,127.8,128.6,128.9,129.4,137.2,140.3$, 154.3, 171.5. HRMS (EI) $\mathrm{m} / z$ calcd for $\mathrm{C}_{18} \mathrm{H}_{17} \mathrm{NO}$ : 263.1310, found 263.1310 .

\section{Synthesis of Rolipram (Scheme 2).}

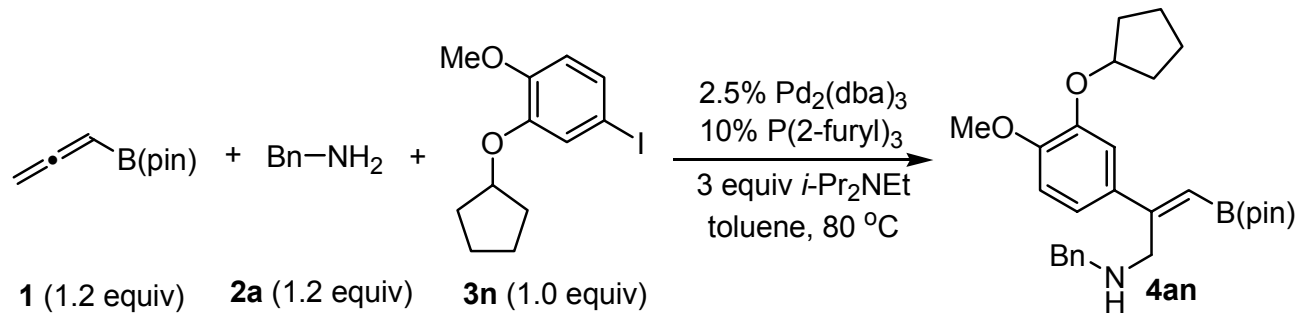

A mixture of $\mathrm{Pd}_{2}(\mathrm{dba})_{3}(6.9 \mathrm{mg}, 7.5 \mu \mathrm{mol}), \mathrm{P}(2$-furyl $) 3(7.0 \mathrm{mg}, 30 \mu \mathrm{mol})$, in dry toluene $(0.5 \mathrm{~mL})$ was stirred at room temperature for $20 \mathrm{~min}$ under argon. To this mixture were added 2-cyclopentyloxy-4-iodo-1-methoxybenzene ${ }^{4}(\mathbf{3 n} ; 94.8 \mathrm{mg}, 0.30 \mathrm{mmol})$, allenylboronate pinacol ester (1; $59.8 \mathrm{mg}, 0.36 \mathrm{mmol}$ ), benzylamine (2a; $38.6 \mathrm{mg}, 0.36 \mathrm{mmol}), i$-Pr $2 \mathrm{NEt}$ (116.3 mg, $0.90 \mathrm{mmol}$ ), and dry toluene $(1.0 \mathrm{~mL})$ and the resultant mixture was further stirred at $80{ }^{\circ} \mathrm{C}$ for $24 \mathrm{~h}$. After cooling the reaction mixture to room temperature, catalyst and salts were removed by filtration (toluene). The filtrate was evaporated to give a crude 4an, which was directly used for the next operation: ${ }^{1} \mathrm{H}$ NMR $\left(400 \mathrm{MHz}, \mathrm{CDCl}_{3}\right) \delta 1.28(\mathrm{~s}, 12 \mathrm{H}), 1.55-1.64(\mathrm{~m}, 2 \mathrm{H}), 1.76-1.96(\mathrm{~m}, 6 \mathrm{H}), 3.66(\mathrm{~s}, 2 \mathrm{H}), 3.80(\mathrm{~s}, 3 \mathrm{H}), 4.00(\mathrm{~s}$, $2 \mathrm{H}), 4.73-4.78(\mathrm{~m}, 1 \mathrm{H}), 6.20(\mathrm{~s}, 1 \mathrm{H}), 6.65(\mathrm{dd}, J=8.4,2.0 \mathrm{~Hz}, 1 \mathrm{H}), 6.71(\mathrm{~d}, J=8.4 \mathrm{~Hz}, 1 \mathrm{H}), 7.02(\mathrm{~d}, J=2.0$ $\mathrm{Hz}, 1 \mathrm{H}), 7.26-7.34(\mathrm{~m}, 2 \mathrm{H}), 7.35-7.43(\mathrm{~m}, 3 \mathrm{H}) .{ }^{13} \mathrm{C} \mathrm{NMR}\left(100 \mathrm{MHz}, \mathrm{CDCl}_{3}\right) \delta 24.1,25.6,32.8,52.3,52.8$, 56.0, 79.8, 80.4, 111.2, 112.6, 116.9, 128.3, 128.4, 129.1, 130.1, 135.7, 142.1, 147.3, 149.6. HRMS (FAB) $\mathrm{m} / \mathrm{z}$ calcd for $\mathrm{C}_{28} \mathrm{H}_{38} \mathrm{BNO}_{4}$ : 464.2972, found 464.2975. 

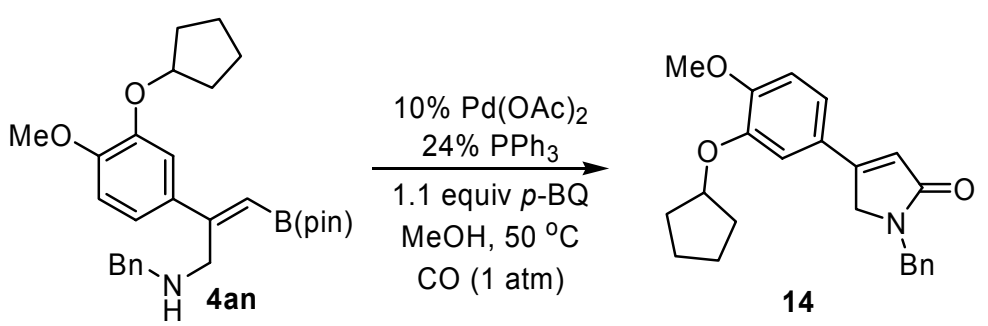

A dry schlenk tube was charged with $\mathrm{Pd}(\mathrm{OAc})_{2}(6.7 \mathrm{mg}, 30 \mu \mathrm{mol}, 10 \mathrm{~mol} \%), \mathrm{PPh}_{3}(19.2 \mathrm{mg}, 73.2$ $\mu \mathrm{mol}, 24 \mathrm{~mol} \%)$, and $p$-benzoquinone $(35.7 \mathrm{mg}, 0.33 \mathrm{mmol}$ ). Then the tube was flushed with carbon monoxide. To the mixture was added a solution of $4 \mathrm{an}$ in anhydrous $\mathrm{MeOH}(3 \mathrm{~mL})$, and then the solution was stirred at $50{ }^{\circ} \mathrm{C}$ for $10 \mathrm{~h}$ under atmospheric pressure of carbon monoxide. The reaction mixture was filtered, and the filtrate was evaporated. Water was added to the residue, and the aqueous phase was extracted with EtOAc. The combined organic phase was washed with brine and dried over $\mathrm{Na}_{2} \mathrm{SO}_{4}$. The filtrate was evaporated, and the residue was subjected to silica gel chromatography (hexane/EtOAc $=2 / 1$ to $1 / 1)$ to afford $14\left(63.4 \mathrm{mg}, 59 \%\right.$ in 2 steps) as colorless solid: ${ }^{1} \mathrm{H} \mathrm{NMR} \mathrm{(400} \mathrm{MHz,}$ $\left.\mathrm{CDCl}_{3}\right) \delta$ 1.57-1.67 (m, 2H), 1.78-1.97 (m, 6H), $3.85(\mathrm{~s}, 3 \mathrm{H}), 4.19(\mathrm{~d}, J=0.8 \mathrm{~Hz}, 2 \mathrm{H}), 4.68(\mathrm{~s}, 2 \mathrm{H}), 4.75-4.79$ $(\mathrm{m}, 1 \mathrm{H}), 6.33(\mathrm{t}, J=0.8 \mathrm{~Hz}, 1 \mathrm{H}), 6.80-6.82(\mathrm{~m}, 1 \mathrm{H}), 6.95-6.97(\mathrm{~m}, 2 \mathrm{H}), 7.25-7.36(\mathrm{~m}, 5 \mathrm{H}) .{ }^{13} \mathrm{C}$ NMR $(100$ $\left.\mathrm{MHz}_{2} \mathrm{CDCl}_{3}\right) \delta 24.1,32.9,46.0,51.9,56.1,80.8,111.6,112.5,118.1,118.8,124.6,127.4,127.7,128.6,137.3$, 147.7, 151.8, 154.3, 171.7. HRMS (EI) $\mathrm{m} / z$ calcd for $\mathrm{C}_{23} \mathrm{H}_{25} \mathrm{NO}_{3}: 363.1834$, found 363.1840 .<smiles>COc1ccc(C2=CC(=O)N(Cc3ccccc3)C2)cc1OC1CCCC1</smiles><smiles>CCO[C@H](C)[C@H](C)OC1CCCC1</smiles>

A mixture of 14 and $\mathrm{Pd} / \mathrm{C}(40 \mathrm{mg}, 5 \% \mathrm{Pd})$ in ethanol $(2.5 \mathrm{~mL})$ was stirred at $60{ }^{\circ} \mathrm{C}$ for $24 \mathrm{~h}$ under $\mathrm{H}_{2}$ (60 atm). The reaction mixture was passed through a pad of Celite, and the filtrate was evaporated to give a crude 15, which was directly used for the next operation; ${ }^{1} \mathrm{H}$ NMR $\left(400 \mathrm{MHz}, \mathrm{CDCl}_{3}\right) \delta 1.55-1.65$ $(\mathrm{m}, 2 \mathrm{H}), 1.76-1.94(\mathrm{~m}, 6 \mathrm{H}), 2.58(\mathrm{dd}, J=16.8,8.4 \mathrm{~Hz}, 1 \mathrm{H}), 2.86(\mathrm{dd}, J=16.8,8.8 \mathrm{~Hz}, 1 \mathrm{H}), 3.24(\mathrm{dd}, J=9.6$, $7.2 \mathrm{~Hz}, 1 \mathrm{H}), 3.42-3.50(\mathrm{~m}, 1 \mathrm{H}), 3.60(\mathrm{dd}, J=9.6,8.4 \mathrm{~Hz}, 1 \mathrm{H}), 3.80(\mathrm{~s}, 3 \mathrm{H}), 4.44(\mathrm{~d}, J=14.4 \mathrm{~Hz}, 1 \mathrm{H}), 4.55(\mathrm{~d}$, $J=14.4 \mathrm{~Hz}, 1 \mathrm{H}), 4.66-4.70(\mathrm{~m}, 1 \mathrm{H}), 6.64-6.68(\mathrm{~m}, 2 \mathrm{H}), 6.76(\mathrm{~d}, J=8.0 \mathrm{~Hz}, 1 \mathrm{H}), 7.24-7.34(\mathrm{~m}, 5 \mathrm{H}) .{ }^{13} \mathrm{C} \mathrm{NMR}$ $\left(100 \mathrm{MHz} \mathrm{CDCl}_{3}\right) \delta 24.13,24.14,32.89,32.91,36.8,39.2,46.7,54.1,56.2,80.6,112.2,113.7,118.6,127.5$, 128.1, 128.6, 134.8, 136.2, 147.8, 149.0, 173.5. HRMS (EI) $\mathrm{m} / \mathrm{z}$ calcd for $\mathrm{C}_{23} \mathrm{H}_{27} \mathrm{NO}_{3}$ : 365.1991, found 365.1991.

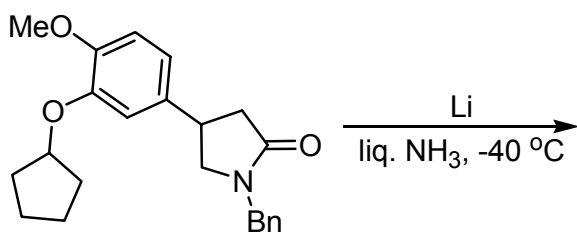

15<smiles>COc1ccc(C2CNC(=O)C2)cc1OC1CCCC1</smiles>

Rolipram

The flask was cooled to $-78{ }^{\circ} \mathrm{C}$ and $\mathrm{NH}_{3}$ gas was then bubbled through the flask till approximately 5 $\mathrm{mL}$ were collected under argon. To the liquid $\mathrm{NH}_{3}$ was added a solution of $\mathbf{1 5}$ in the minimum amount of dry THF, and this solution was allowed to warm to $-40{ }^{\circ} \mathrm{C}$. Excess lithium (bars) was added, and the resulting solution was stirred for $10 \mathrm{~min}$. After solid $\mathrm{NH}_{4} \mathrm{Cl}$ was added to quench the reaction, $\mathrm{NH}_{3}$ was evaporated by allowing the reaction mixture to warm till room temperature. Water was added to the residue, and the aqueous phase was extracted with $\mathrm{CHCl}_{3}$. The combined organic phase was washed with brine and dried over $\mathrm{Na}_{2} \mathrm{SO}_{4}$. The filtrate was evaporated, and the residue was subjected to silica gel chromatography (hexane/EtOAc $=1 / 5$ to EtOAc only) to afford rolipram ${ }^{5}(20.8 \mathrm{mg}, 81 \%$ in 2 steps) 
as colorless solid: ${ }^{1} \mathrm{H}$ NMR (400 MHz, $\left.\mathrm{CDCl}_{3}\right) \delta 1.58-1.63(\mathrm{~m}, 2 \mathrm{H}), 1.80-1.94(\mathrm{~m}, 6 \mathrm{H}), 2.47(\mathrm{dd}, J=16.8,9.2$ $\mathrm{Hz}, 1 \mathrm{H}), 2.71(\mathrm{dd}, J=16.8,9.2 \mathrm{~Hz}, 1 \mathrm{H}), 3.38(\mathrm{dd}, J=9.2,7.2 \mathrm{~Hz}, 1 \mathrm{H}), 3.58-3.67(\mathrm{~m}, 1 \mathrm{H}), 3.75(\mathrm{dd}, J=9.2$, $7.2 \mathrm{~Hz}, 1 \mathrm{H}), 3.83(\mathrm{~s}, 3 \mathrm{H}), 4.74-4.78(\mathrm{~m}, 1 \mathrm{H}), 5.90(\mathrm{br}, 1 \mathrm{H}), 6.75-6.83(\mathrm{~m}, 3 \mathrm{H}) .{ }^{13} \mathrm{C}$ NMR $\left(100 \mathrm{MHz}, \mathrm{CDCl}_{3}\right) \delta$ 24.1, 32.9, 38.1, 40.1, 49.7, 56.2, 80.7, 112.3, 113.8, 118.7, 134.4, 147.8, 149.1, 177.2. HRMS (EI) $\mathrm{m} / \mathrm{z}$ calcd for

$\mathrm{C}_{16} \mathrm{H}_{21} \mathrm{NO}_{3}: 275.1521$, found 275.1525.

\section{References Cited in Supporting Information}

(1) (a) Crebbé, P.; Fillion, H.; André, D.; Luche, J.-L. J. Chem. Soc., Chem. Commun. 1979, 859. (b) Searles, S.; Li, Y.; Nassim, B.; Lopes, M.-T. R.; Tran, P. T.; Crabbé, P. J. Chem. Soc., Perkin Trans. 1 1984, 747. (c) Crabbé, P.; Nassim, B.; Lopes, M.-T. R. Organic Synthesis; Wiley: New York, 1990; Collect. Vol. VII, p 276.

(2) Zheng, B.; Myers, A. G. J. Am. Chem. Soc. 1996, 118, 4492. For the preparation of o-nitrobenzenesulfonylhydrazine (NBSH), see: Myers, A. G.; Zheng, B.; Movassaghi, M. J. Org. Chem. $1997,62,7507$.

(3) Hopf, H.; Bohm, I.; Kleinschroth, J. Org. Synth. 1982, 60, 41.

(4) Muraoka, N.; Mineno, M.; Itami, K.; Yoshida, J. J. Org. Chem. 2005, 70, 6933.

(5) Garcia, A. L. L.; Carpes, M. J. S.; de Oca, A. C. B. M.; dos Santos, M. A. G.; Santana, C. C.; Correia, C.

R. D. J. Org. Chem. 2005, 70, 1050. 
${ }^{1} \mathrm{H}$ NMR $\left(400 \mathrm{MHz}, \mathrm{CDCl}_{3}\right)$<smiles>C=CB1OC(C)(C)C(C)(C)O1</smiles>

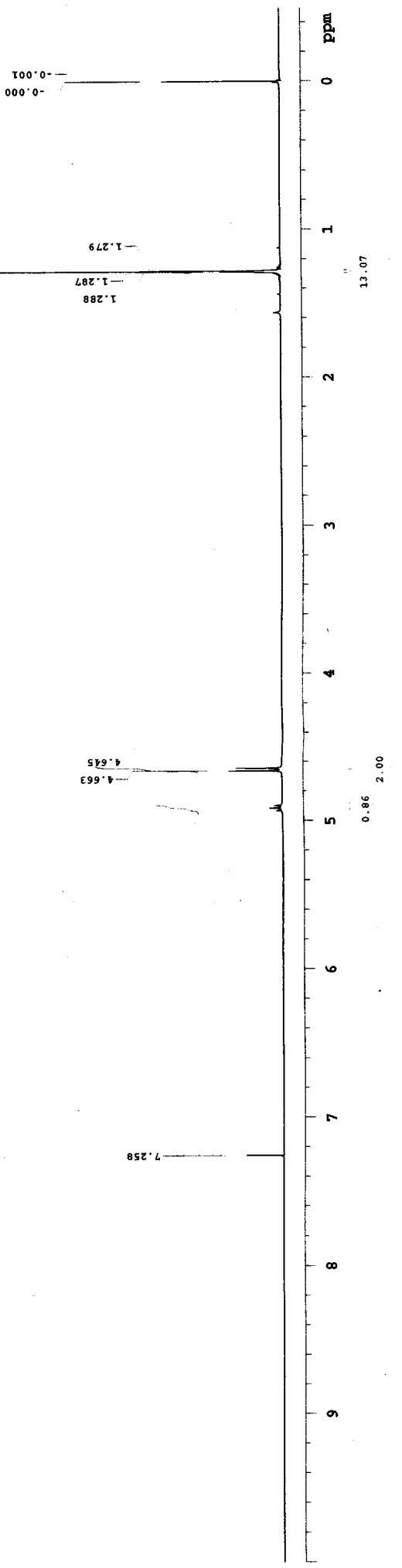


${ }^{13} \mathrm{C}$ NMR (400MHz, $\mathrm{CDCl}_{3}$ )<smiles></smiles>

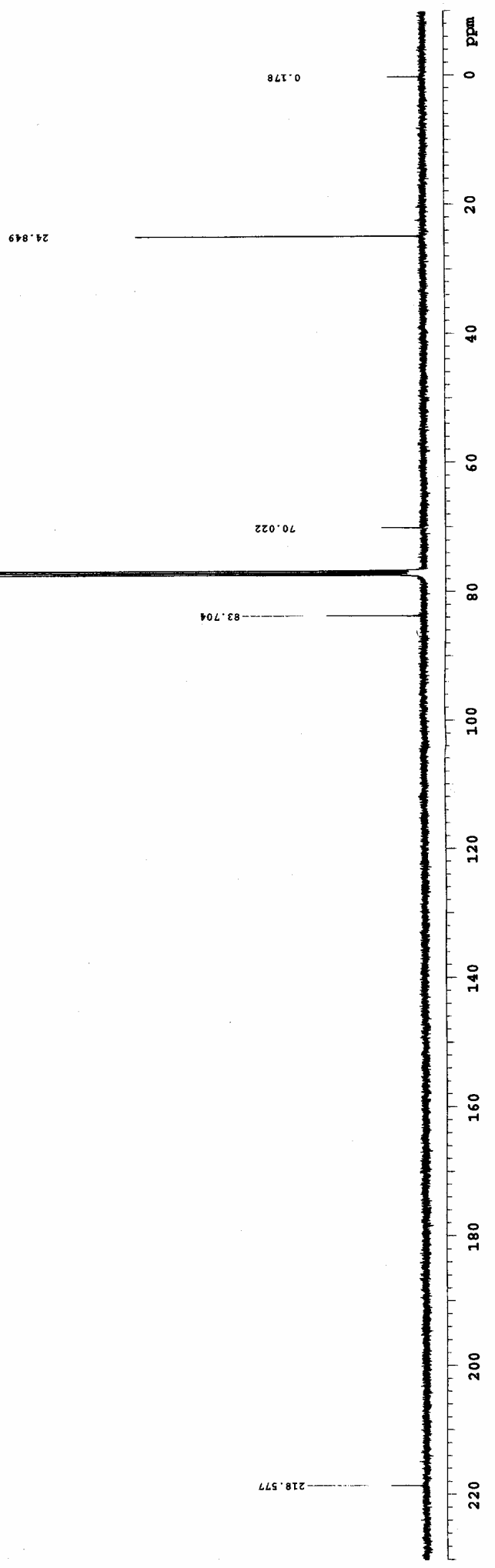


${ }^{1} \mathrm{H}$ NMR $\left(400 \mathrm{MHz}, \mathrm{CDCl}_{3}\right)$
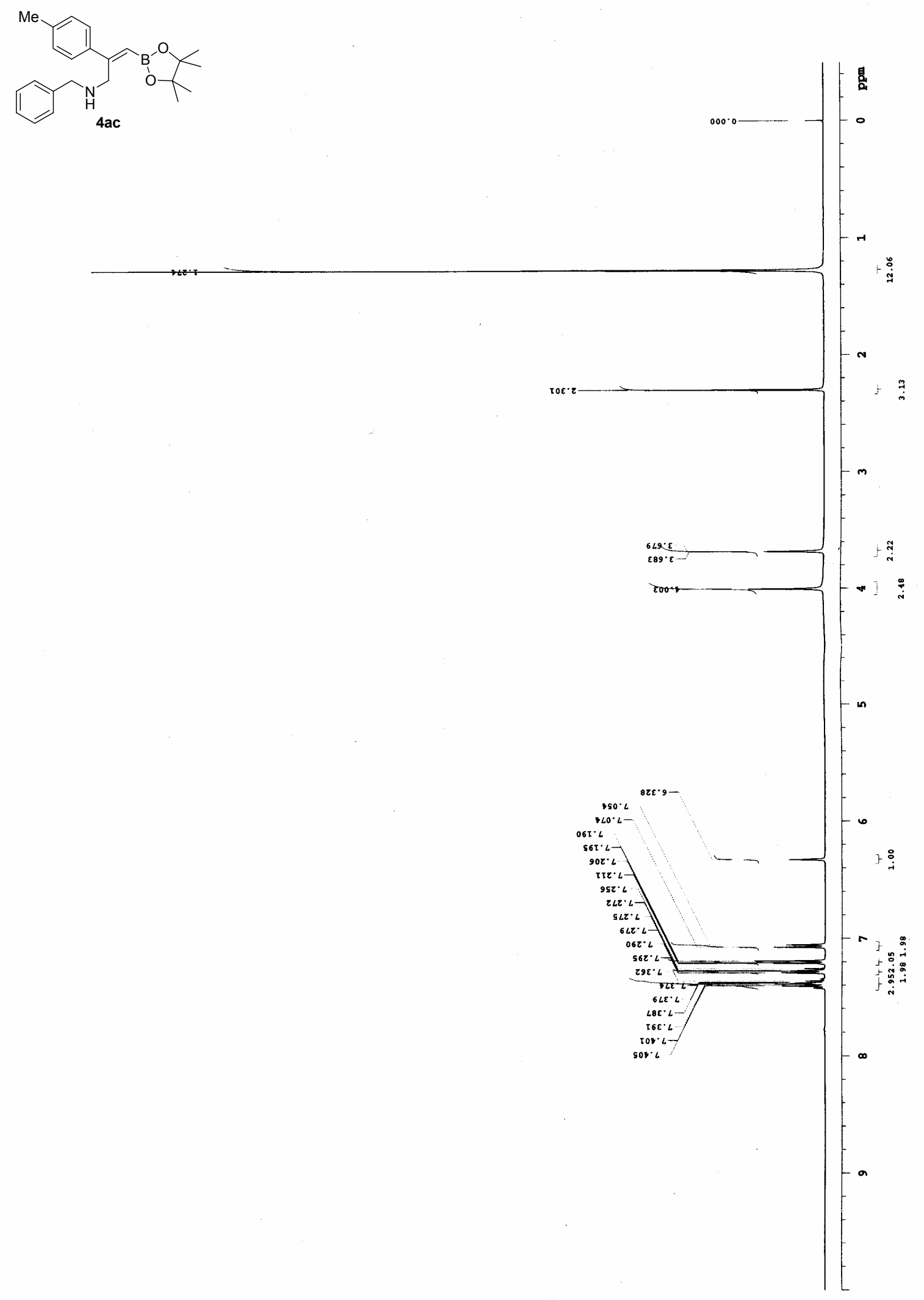
${ }^{13} \mathrm{C}$ NMR (100MHz, $\mathrm{CDCl}_{3}$ )

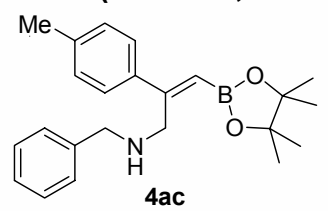

$889^{\circ} 9$

$000 \cdot 4 L$

ZOZ. $L L$
LI $\varepsilon$ LL

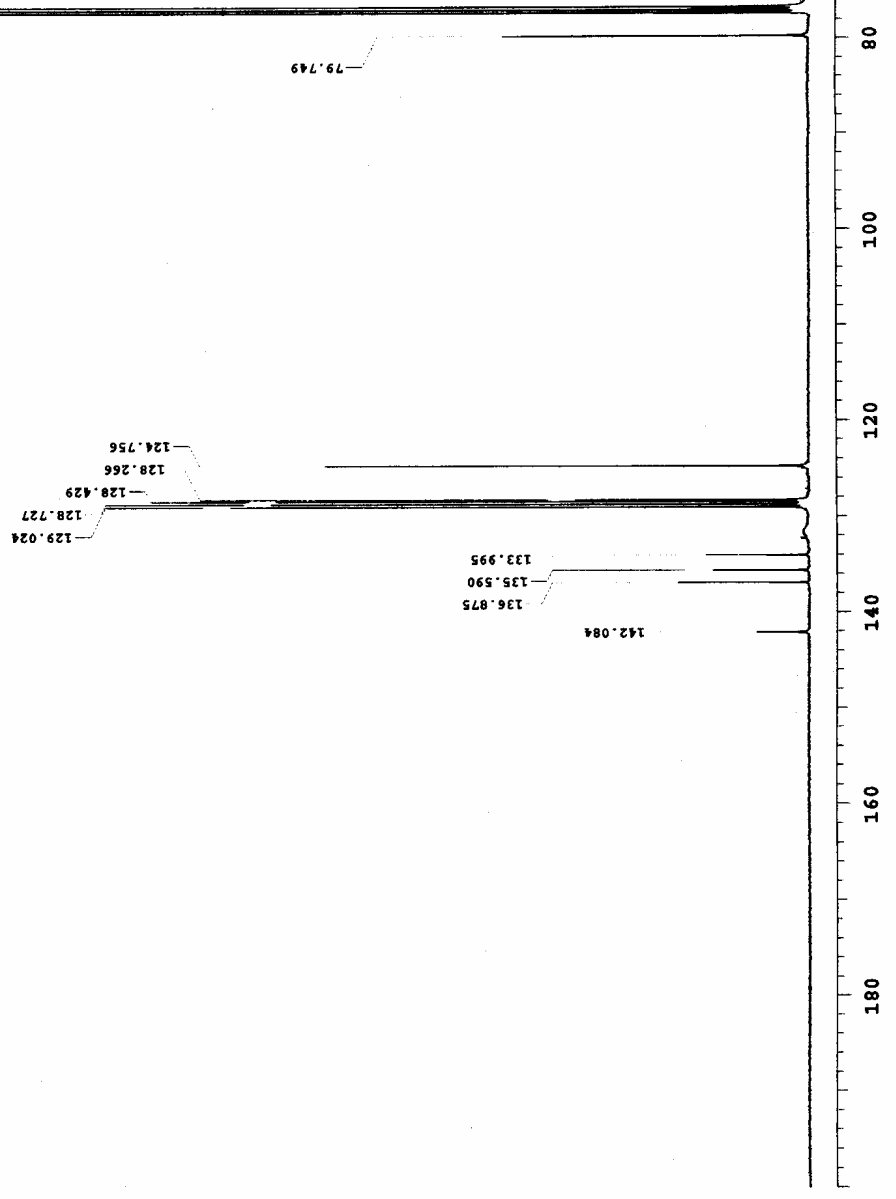


NOESY $\left(400 \mathrm{MHz}, \mathrm{CDCl}_{3}\right)$

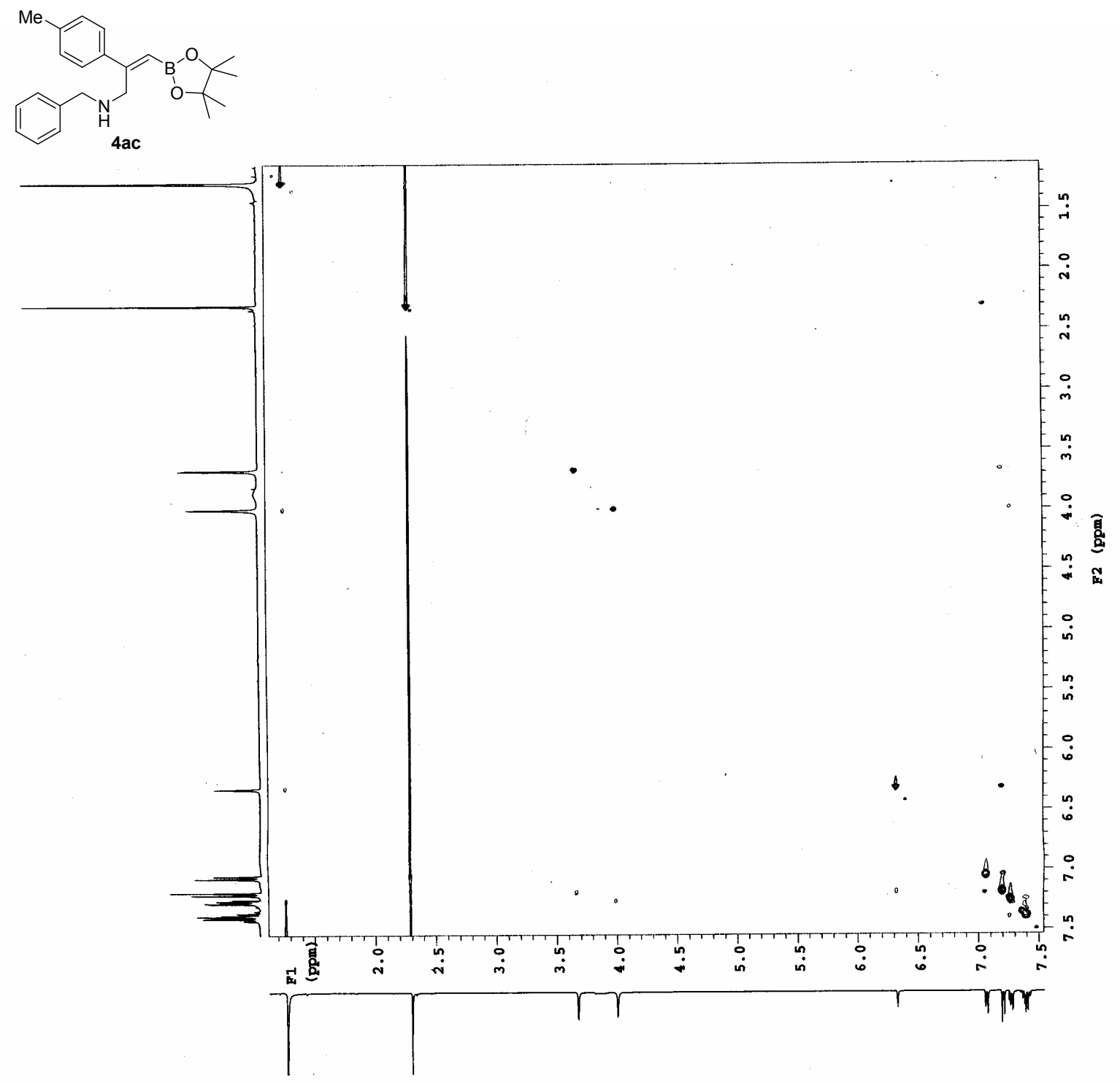


${ }^{1} \mathrm{H}$ NMR (400MHz, $\mathrm{CDCl}_{3}$ )
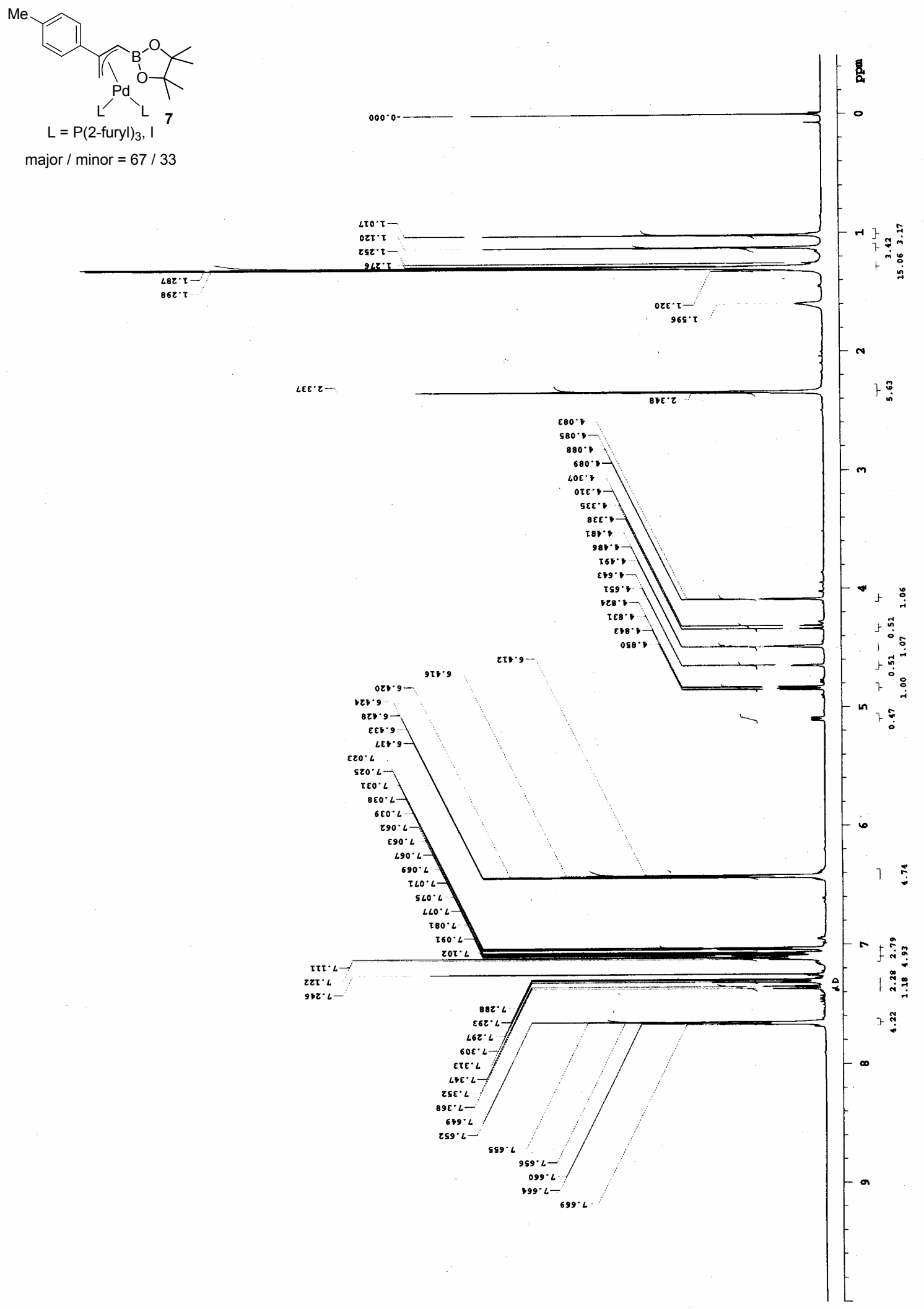
${ }^{13} \mathrm{C} \mathrm{NMR}\left(400 \mathrm{MHz}, \mathrm{CDCl}_{3}\right)$

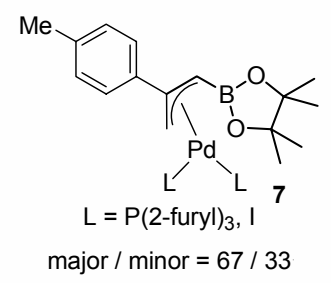

major $/$ minor $=67 / 33$

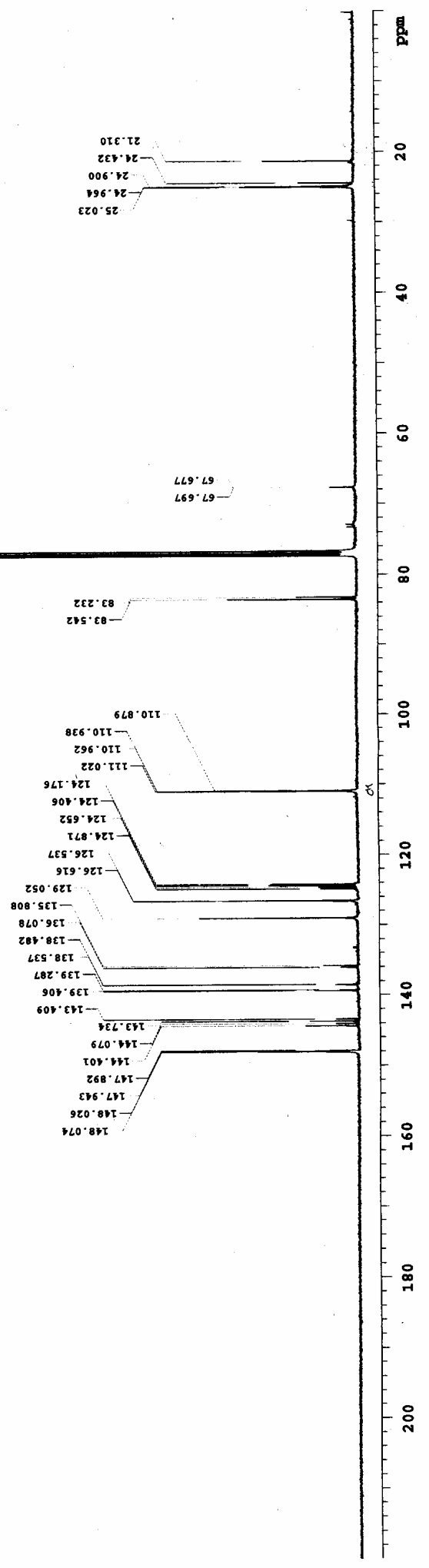


${ }^{1} \mathrm{H}$ NMR $\left(400 \mathrm{MHz}, \mathrm{CDCl}_{3}\right)$
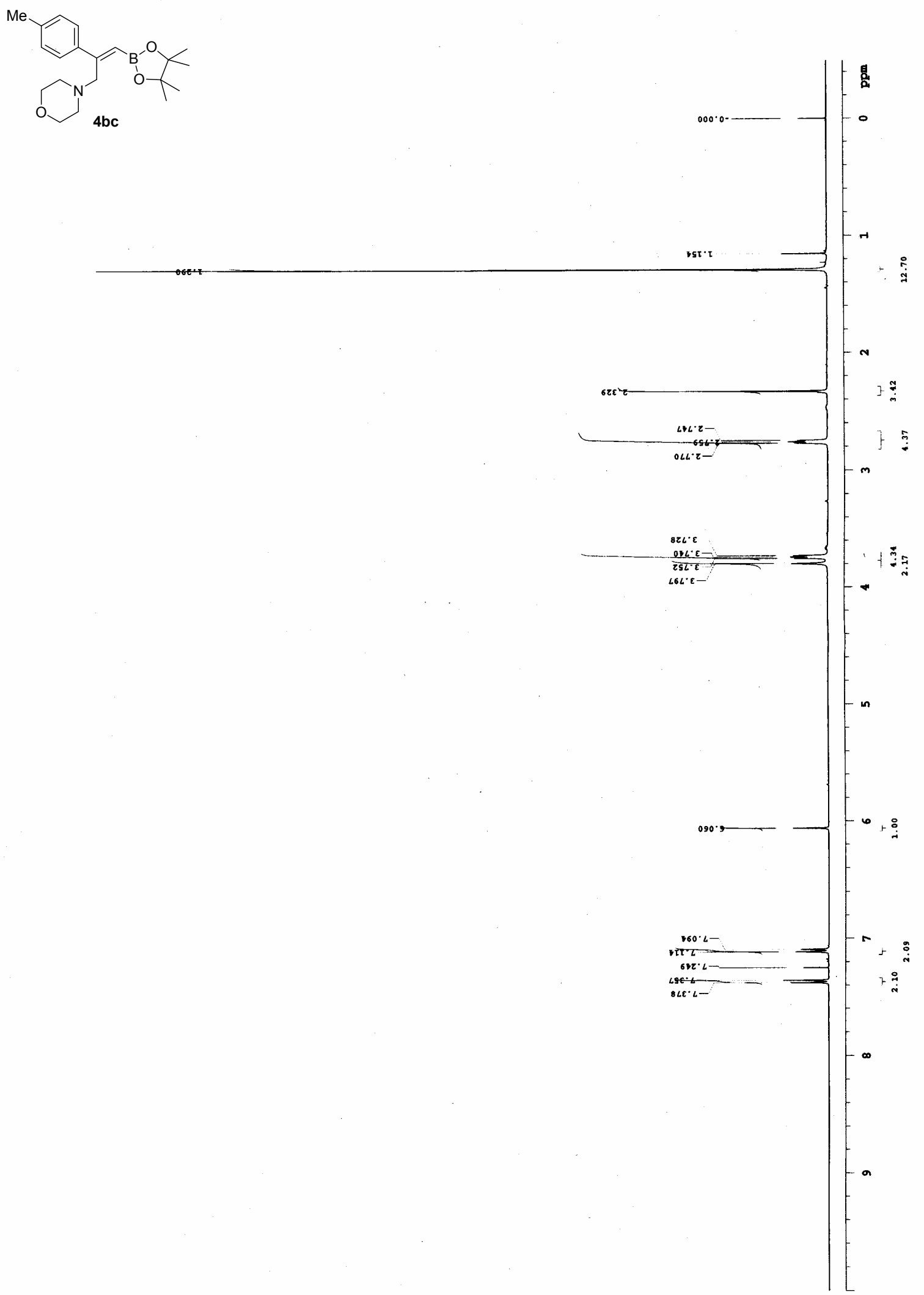
${ }^{13} \mathrm{C} \mathrm{NMR}$ (100MHz, $\mathrm{CDCl}_{3}$ )
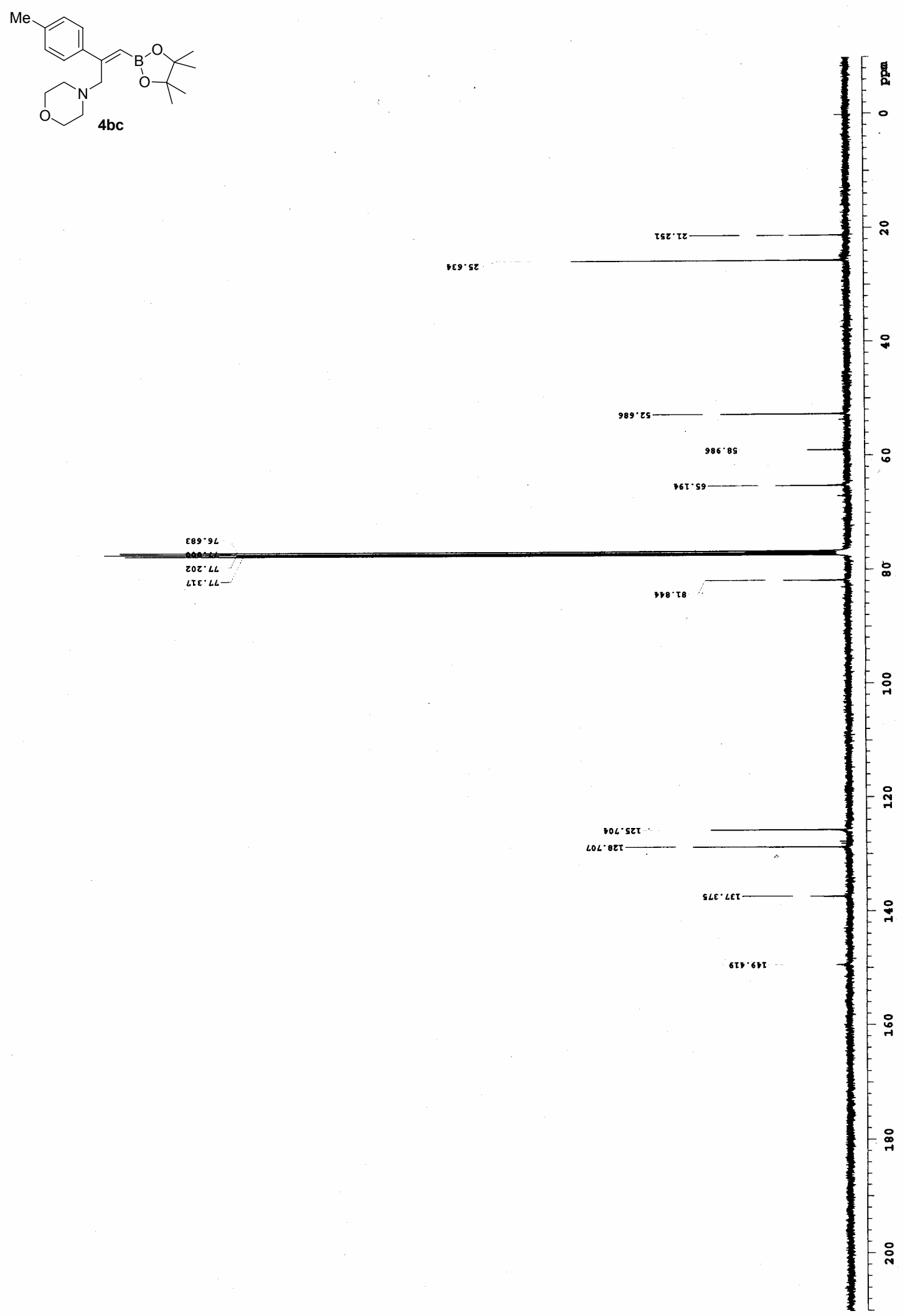
NOESY $\left(400 \mathrm{MHz}, \mathrm{CDCl}_{3}\right)$

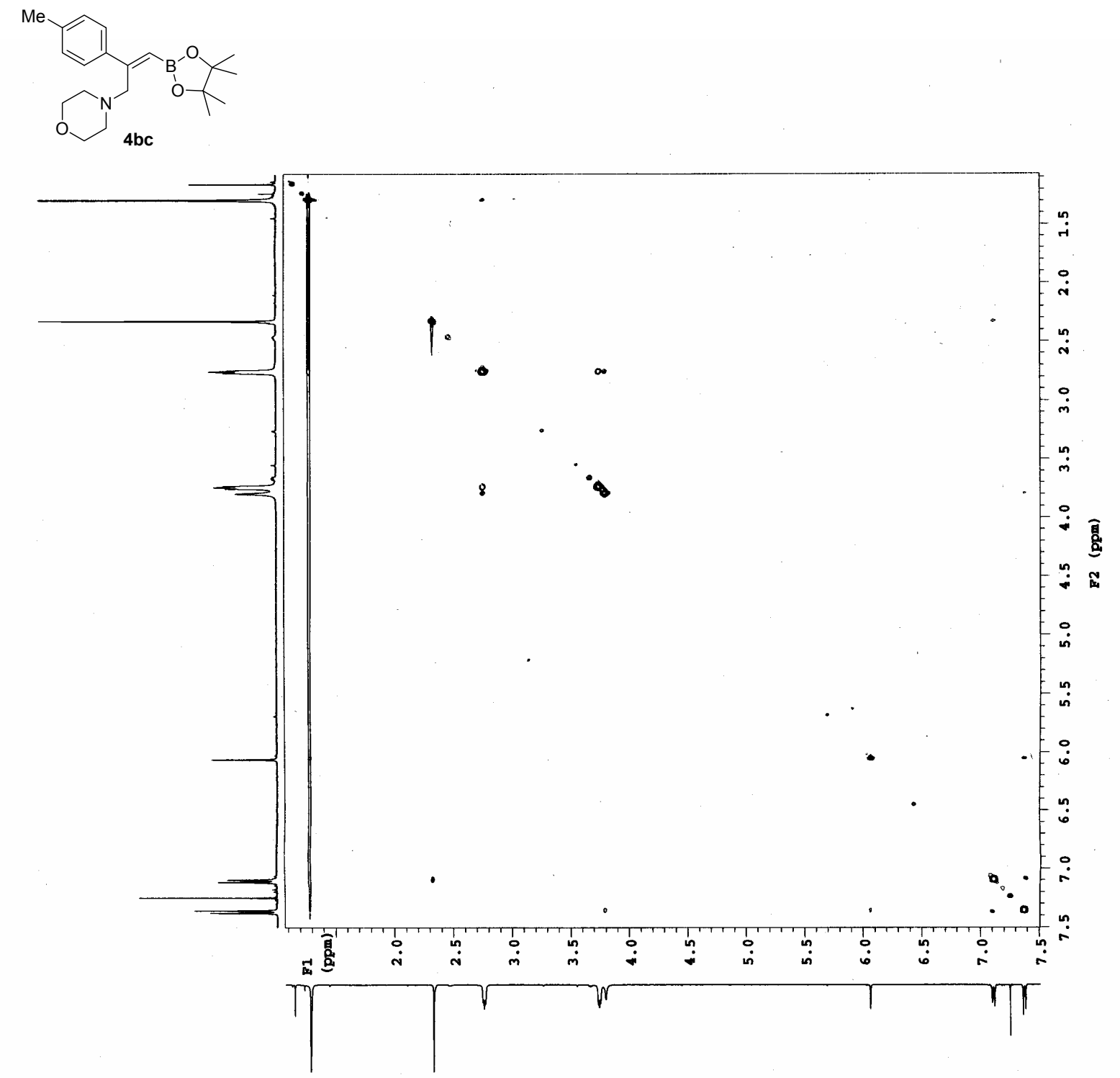


${ }^{1} \mathrm{H}$ NMR $\left(400 \mathrm{MHz}, \mathrm{CDCl}_{3}\right)$

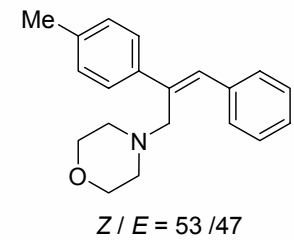

$298: z-$

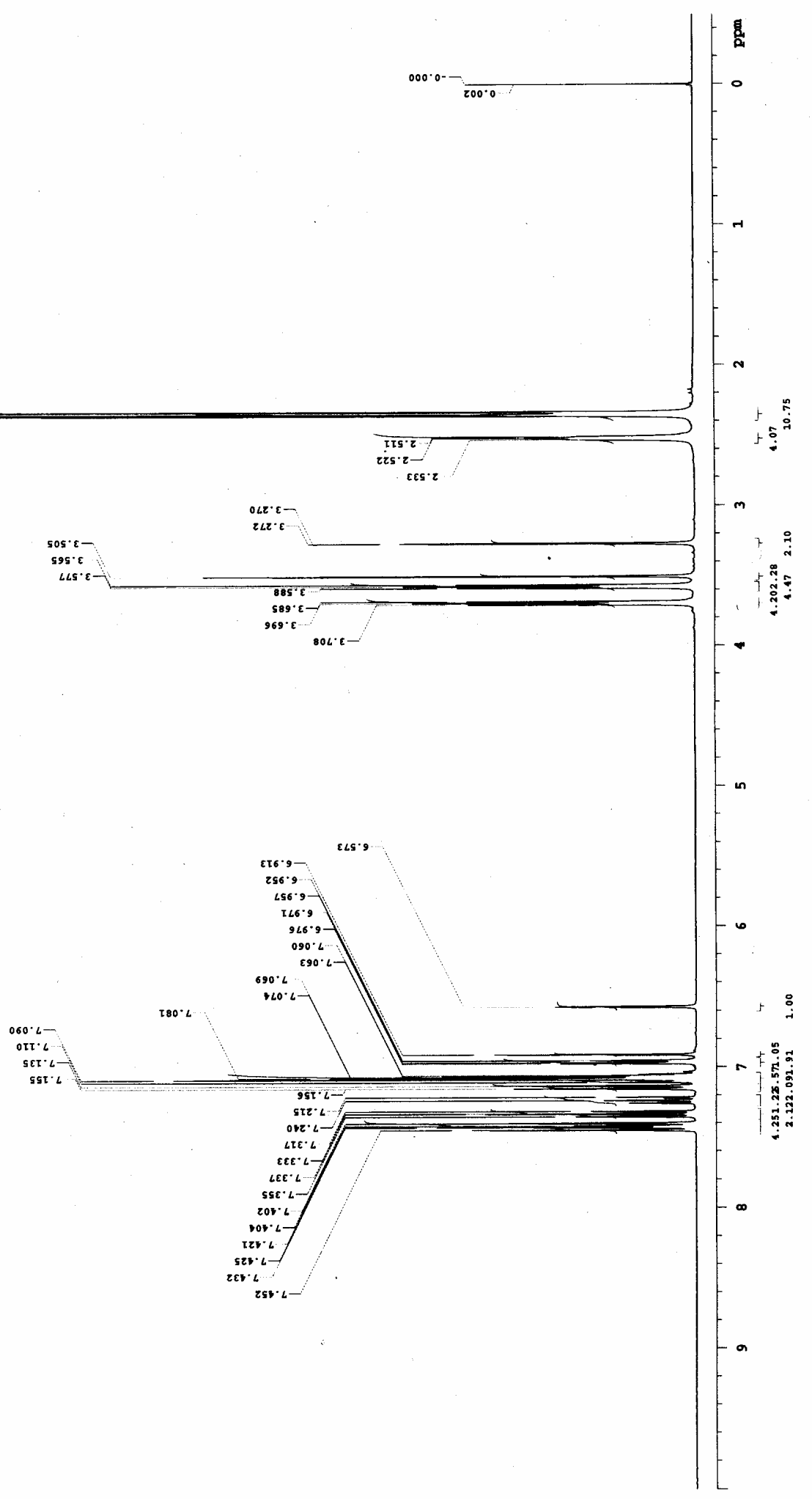


${ }^{13} \mathrm{C} \mathrm{NMR}$ (100MHz, $\mathrm{CDCl}_{3}$ )

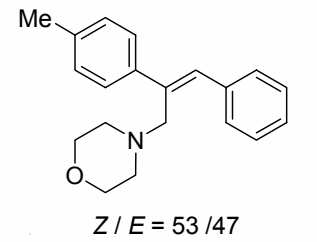

$\varepsilon 89 \cdot 96$

ZIE $\angle L=$

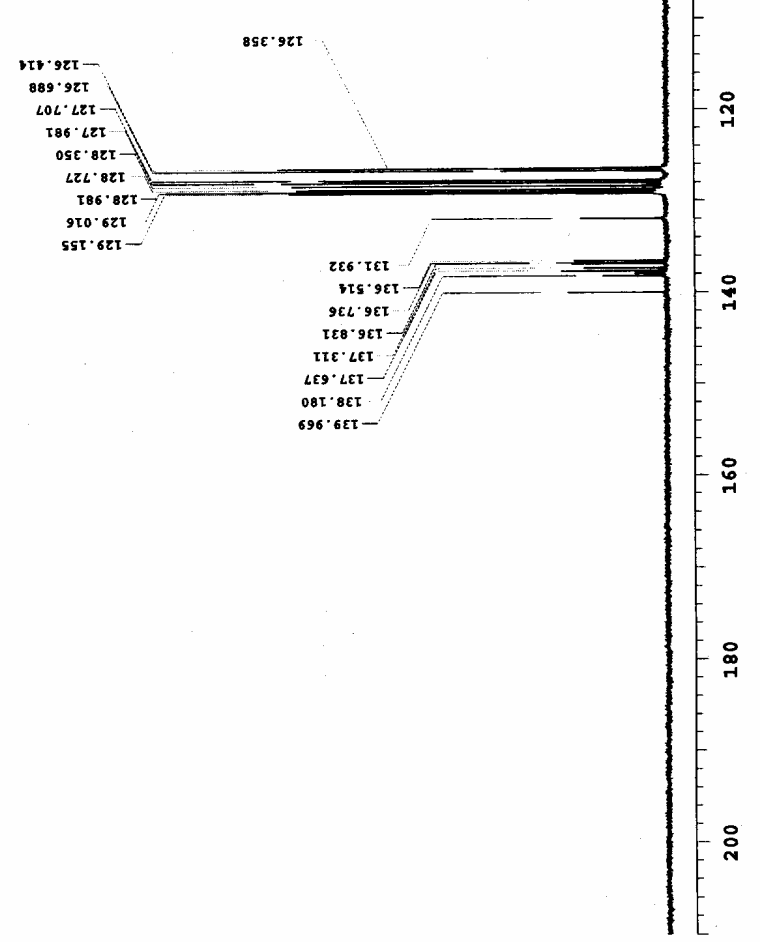


${ }^{1} \mathrm{H}$ NMR $\left(400 \mathrm{MHz}, \mathrm{CDCl}_{3}\right)$

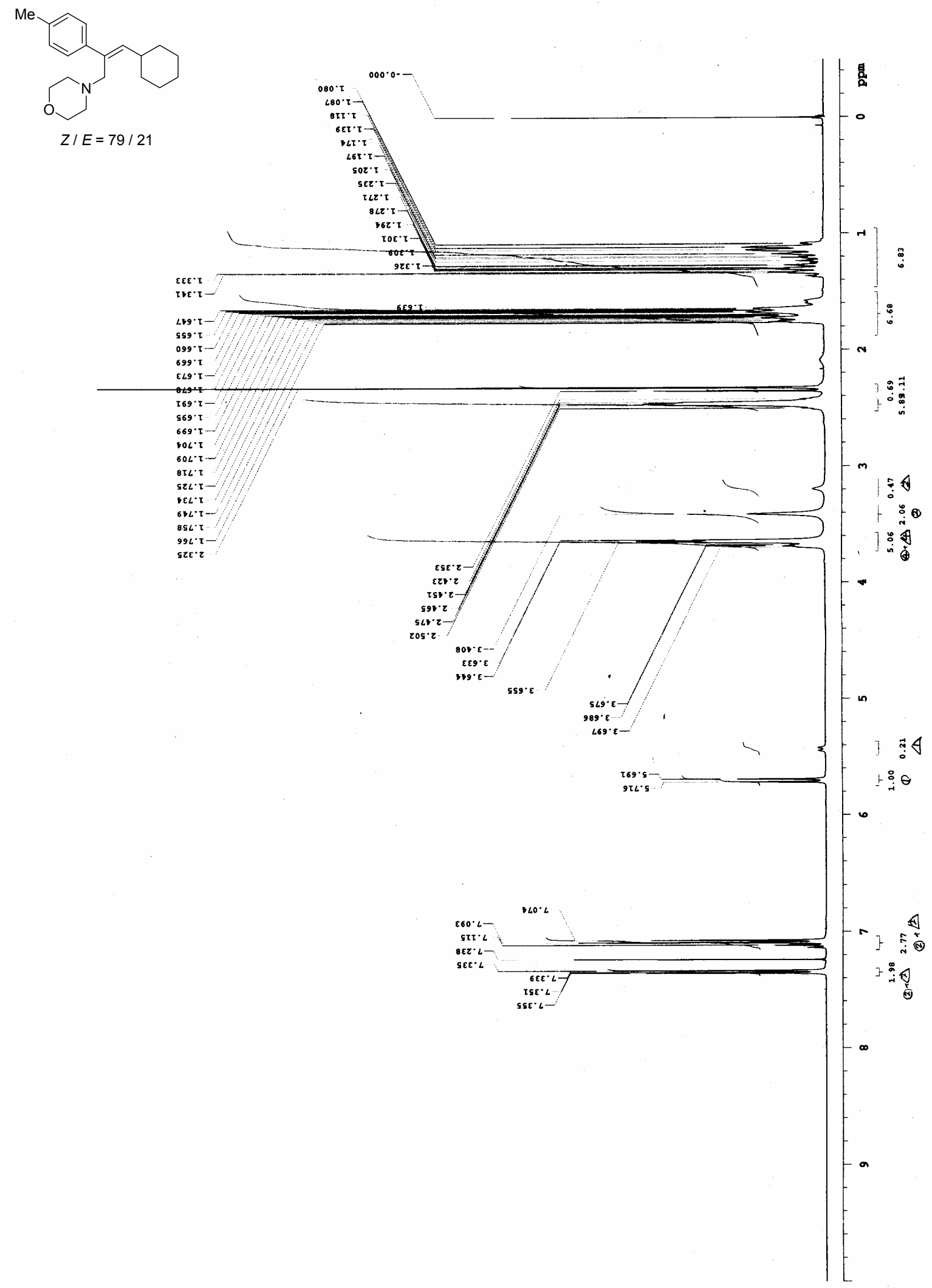


${ }^{13} \mathrm{C} \mathrm{NMR}$ (100MHz, $\mathrm{CDCl}_{3}$ )
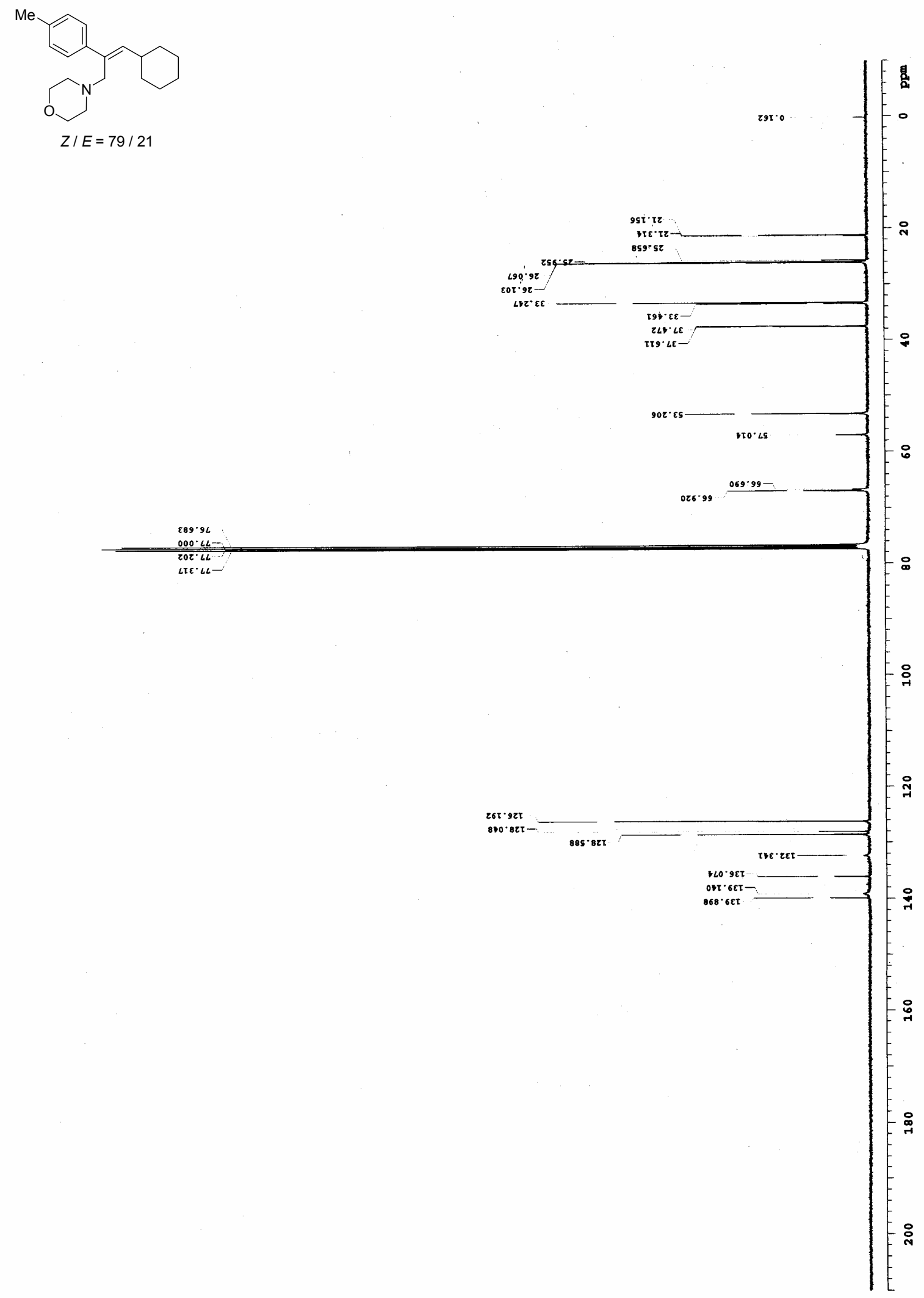
${ }^{1} \mathrm{H}$ NMR $\left(400 \mathrm{MHz}, \mathrm{CDCl}_{3}\right)$<smiles>CC(=O)c1ccc(/C=C(\CN2CCOCC2)c2ccccc2)cc1</smiles>

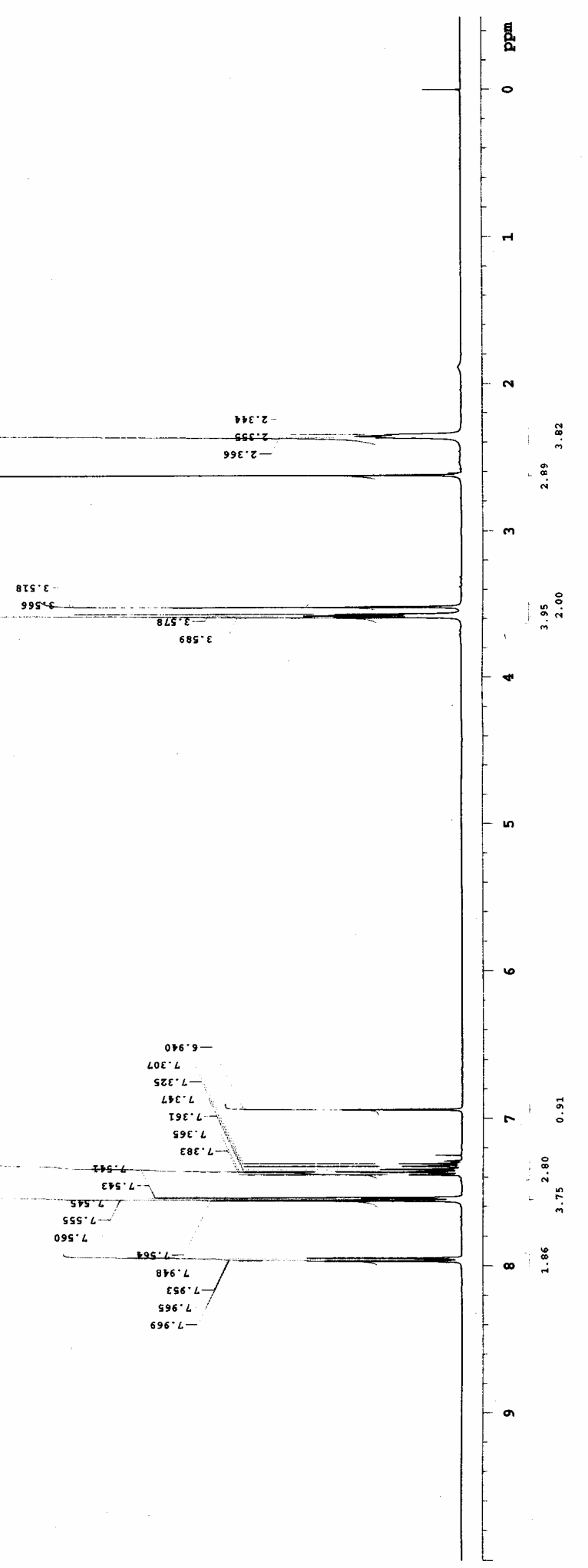


${ }^{13} \mathrm{C} \mathrm{NMR}\left(100 \mathrm{MHz}, \mathrm{CDCl}_{3}\right)$
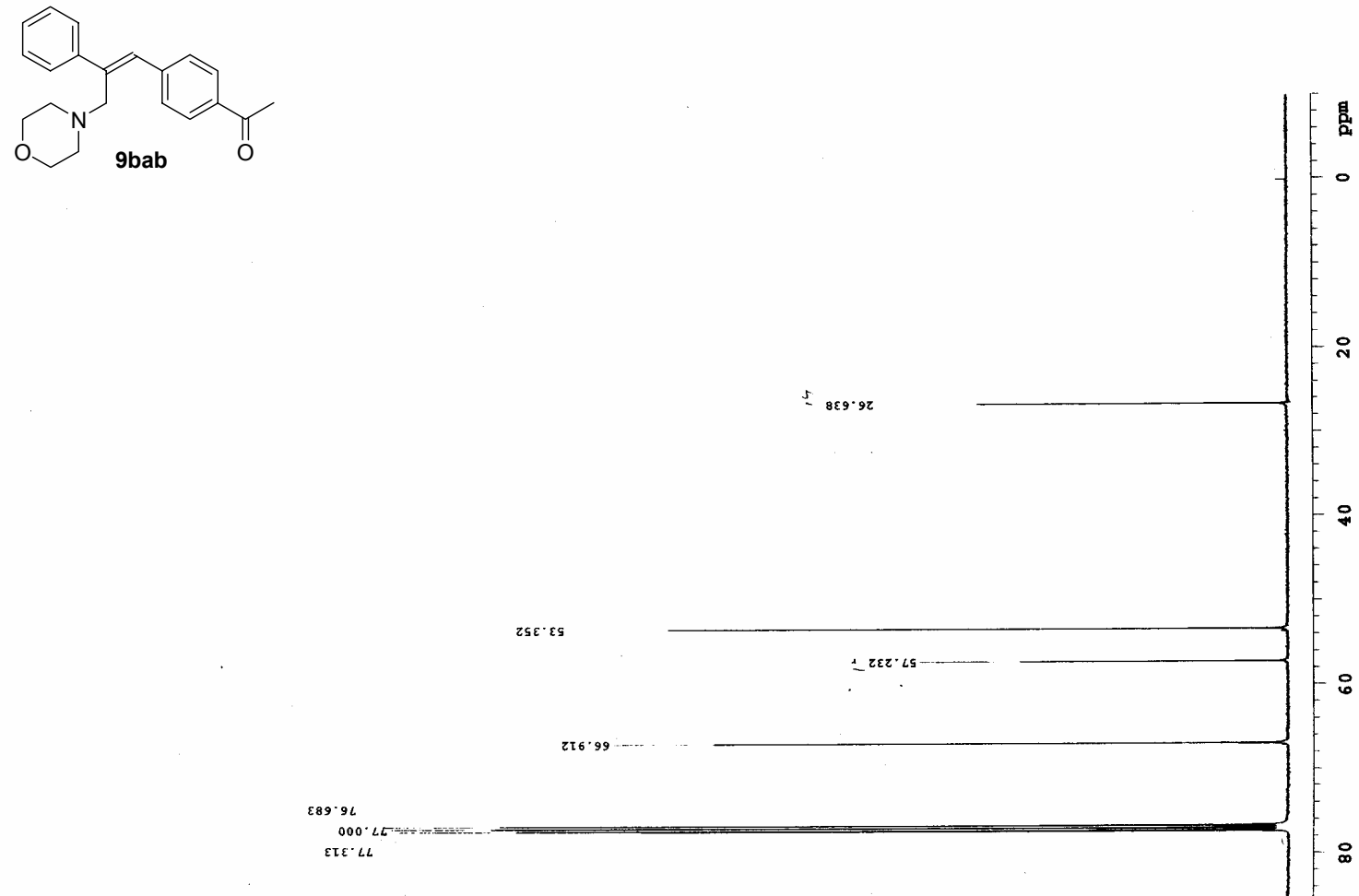
$\tau \bar{T} \cdot 9 z \tau-$
$z 0 \sigma^{\circ} \cdot L z \tau$ $2 z \varepsilon \cdot 6 z \pi$

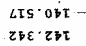

08v-zrt-

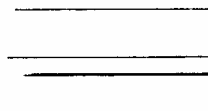


${ }^{1} \mathrm{H}$ NMR $\left(400 \mathrm{MHz}, \mathrm{CDCl}_{3}\right)$
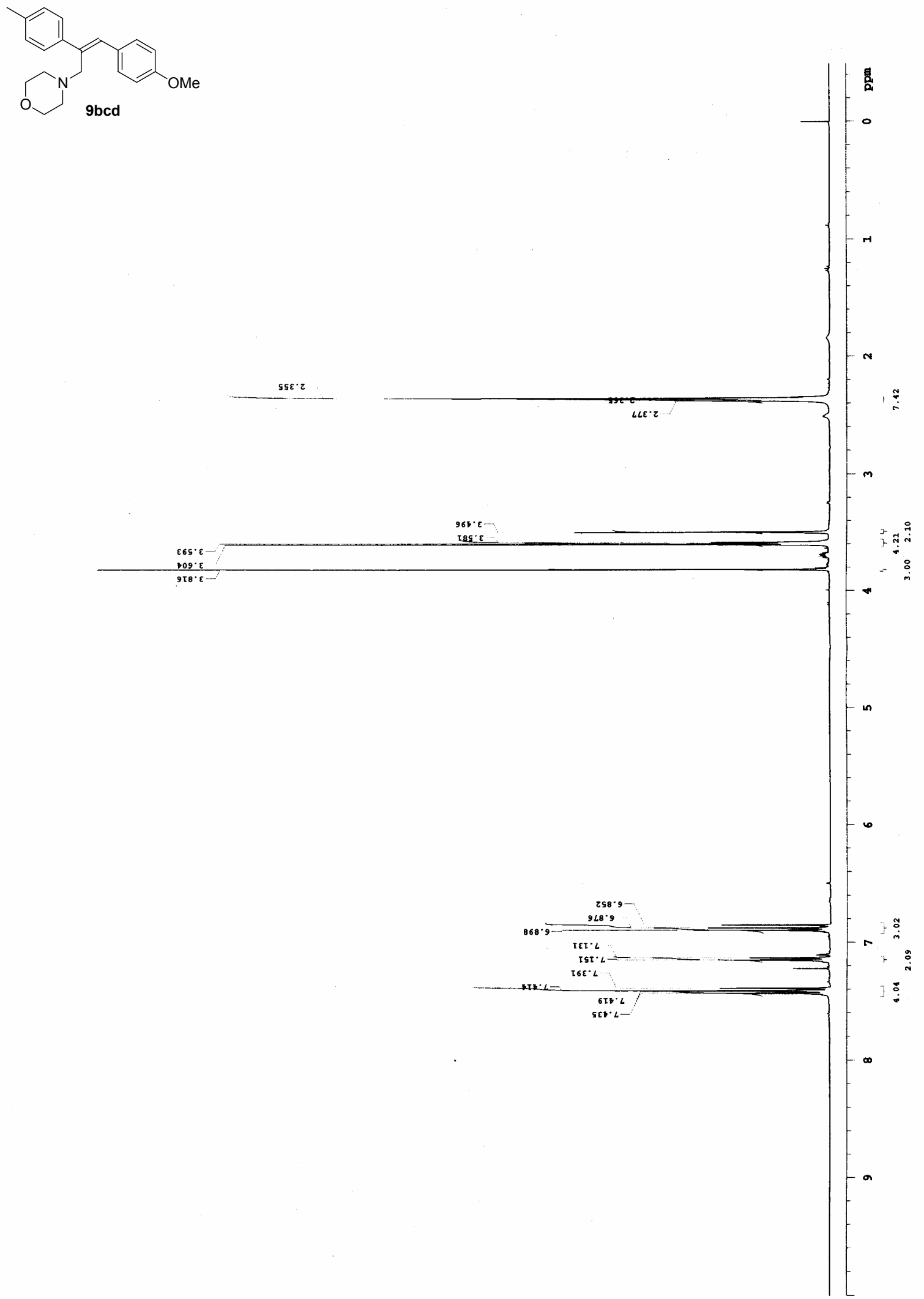
${ }^{13} \mathrm{C}$ NMR (100MHz, $\mathrm{CDCl}_{3}$ )
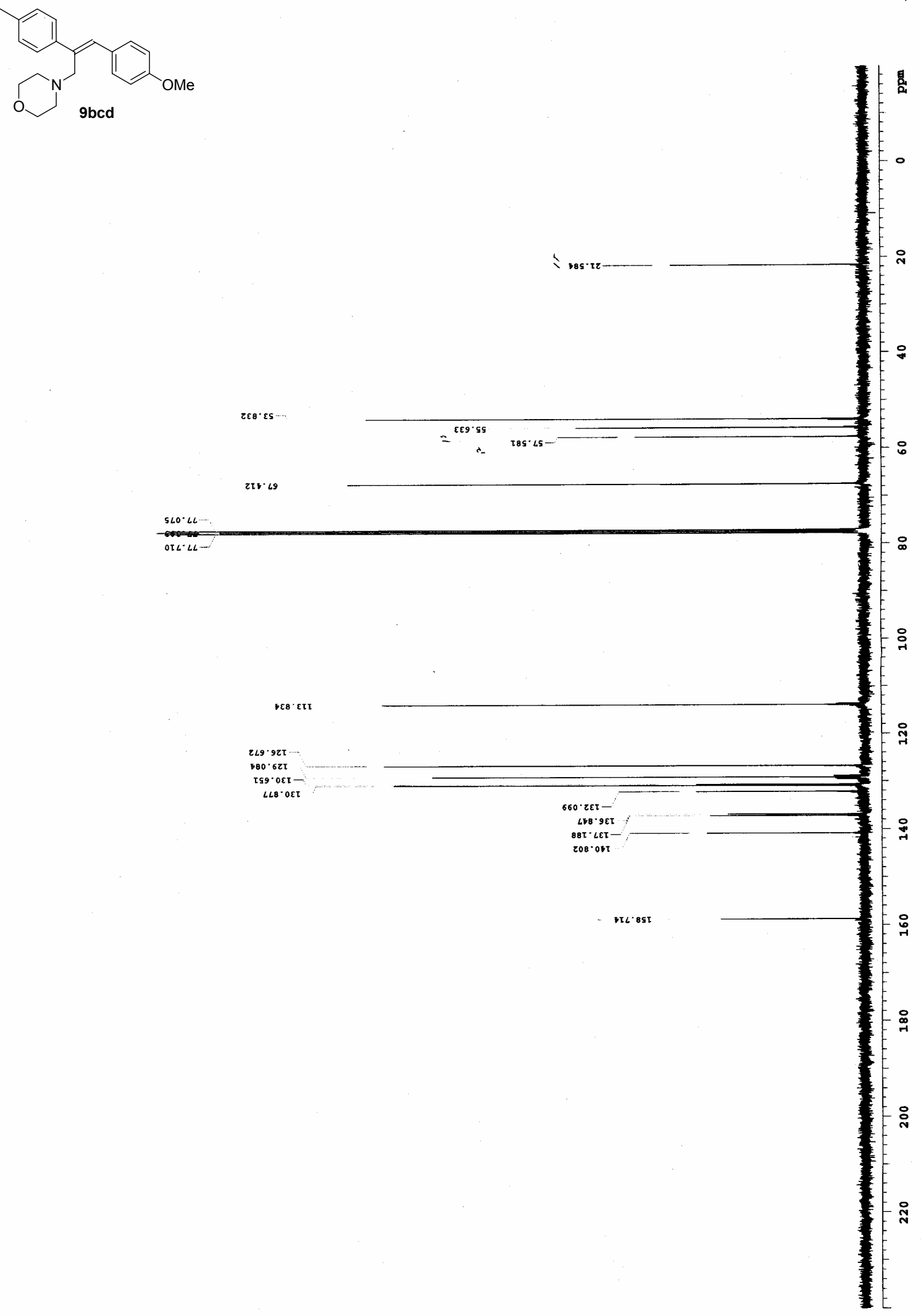
${ }^{1} \mathrm{H}$ NMR (400MHz, $\mathrm{CDCl}_{3}$ )
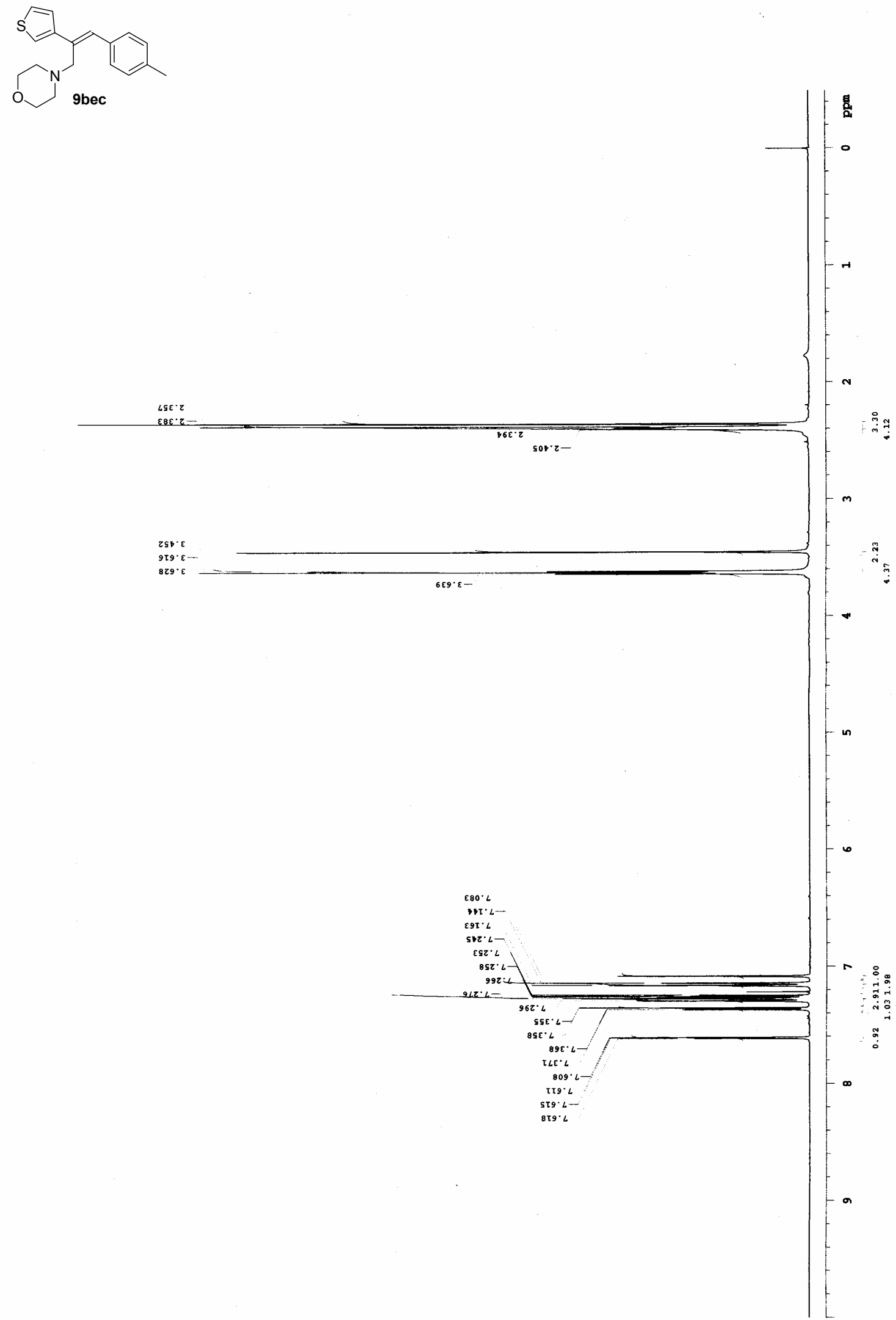
${ }^{13} \mathrm{C}$ NMR (100MHz, $\left.\mathrm{CDCl}_{3}\right)$
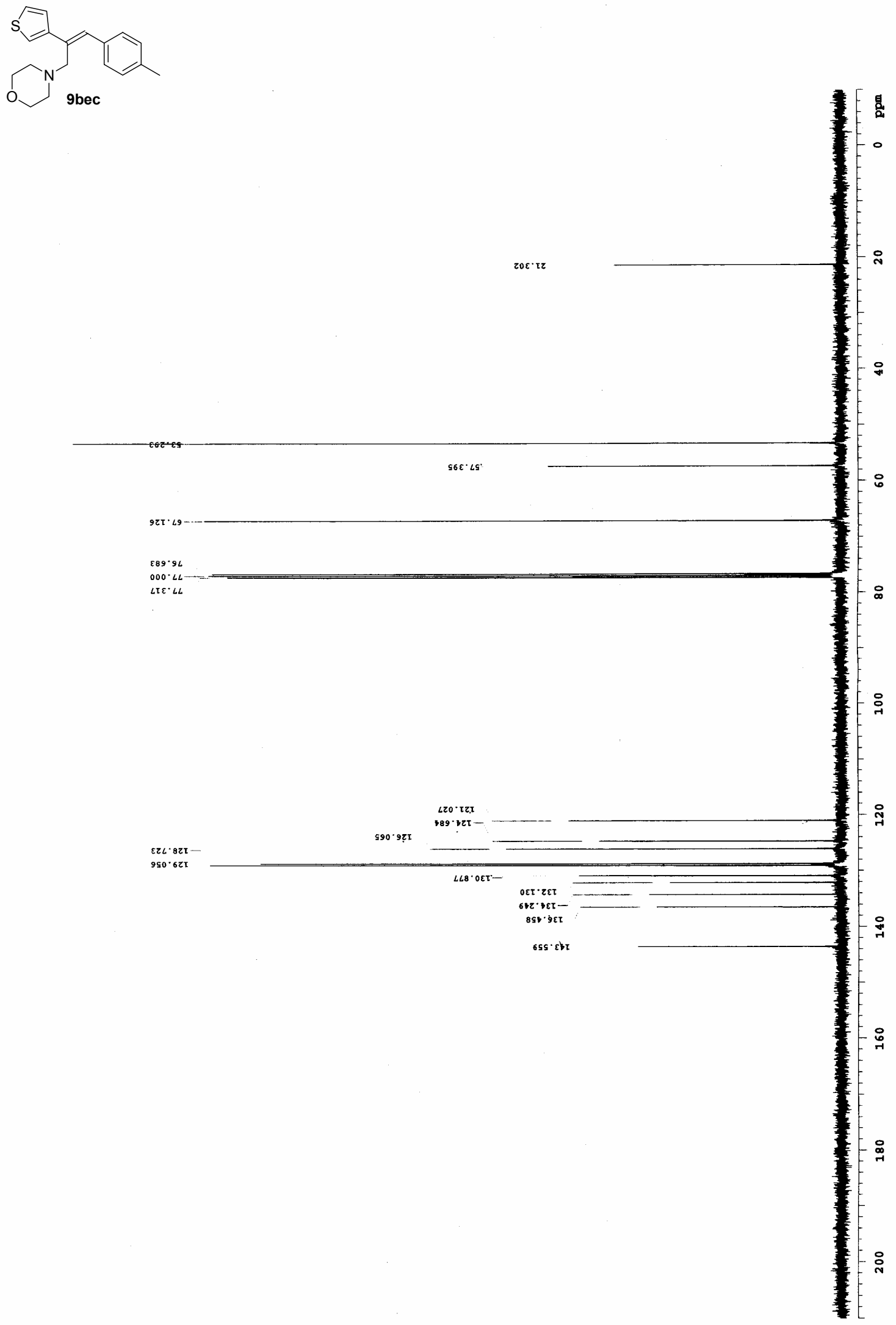
${ }^{1} \mathrm{H}$ NMR $\left(400 \mathrm{MHz}, \mathrm{CDCl}_{3}\right)$<smiles>FC(F)(F)c1ccc(/C(=C/c2ccccc2)CN2CCOCC2)cc1</smiles>

हท5' $\varepsilon$

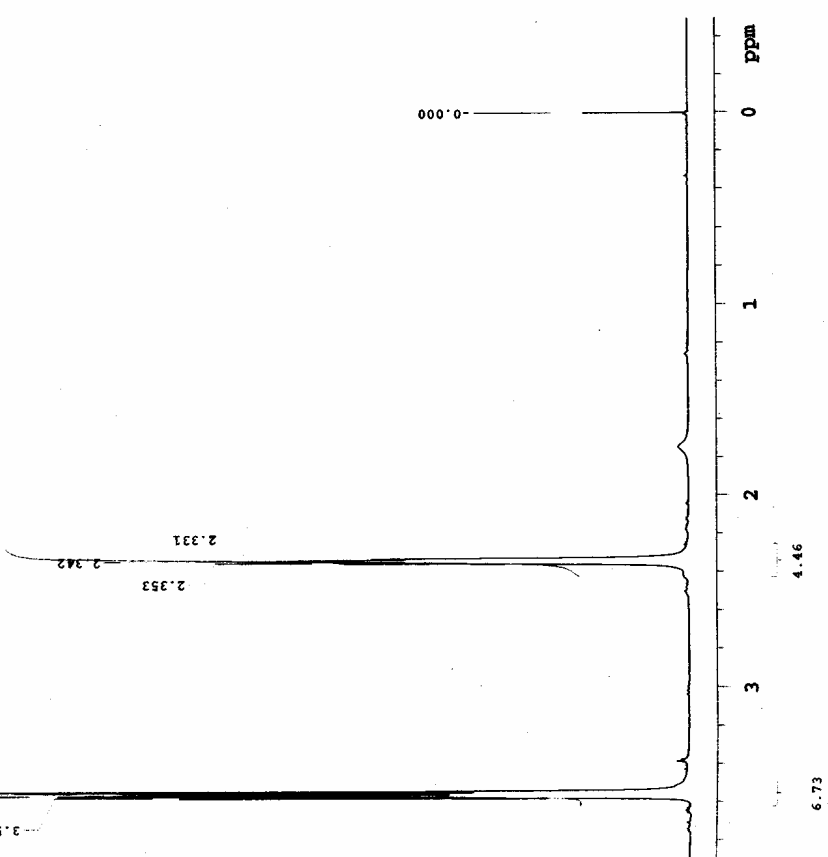

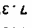

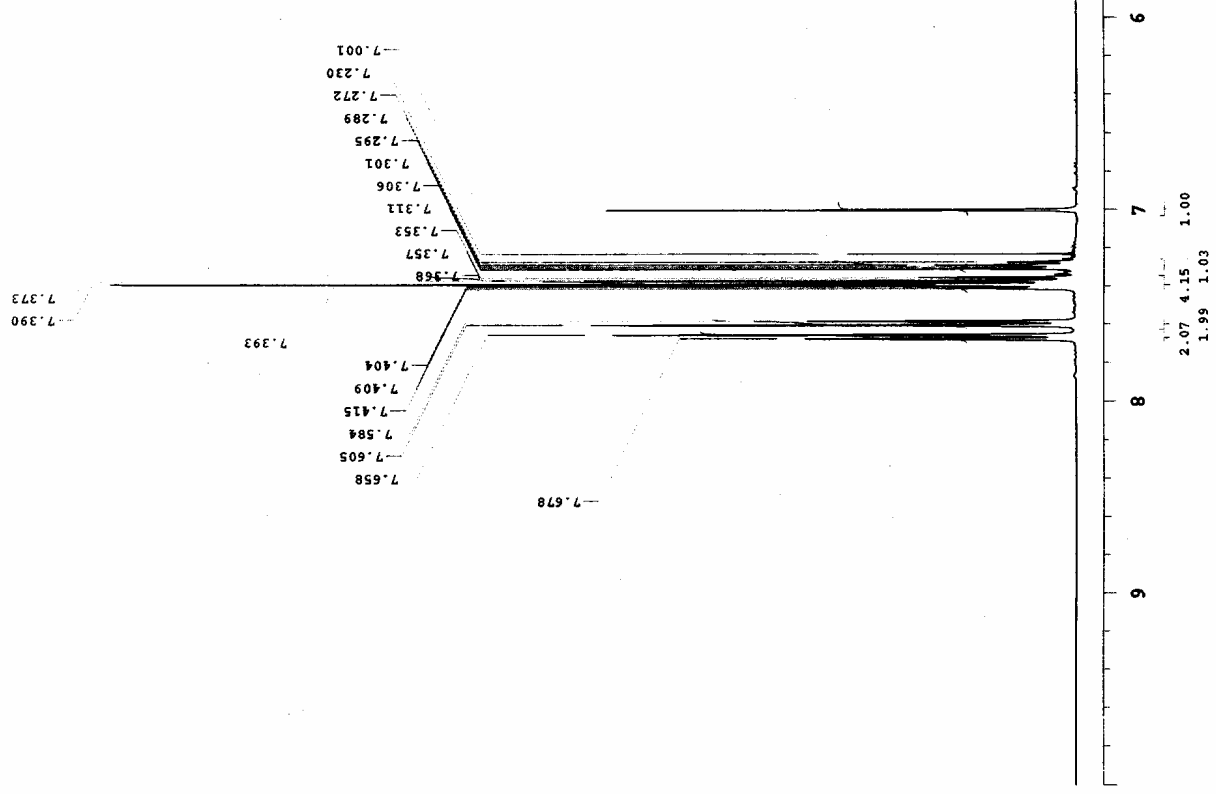


${ }^{13} \mathrm{C} \mathrm{NMR}$ (100MHz, $\mathrm{CDCl}_{3}$ )<smiles>C[C@@H]1COCCN1C/C(=C\c1ccccc1)c1ccc(C(F)(F)F)cc1</smiles><smiles>C1CCCC1</smiles>

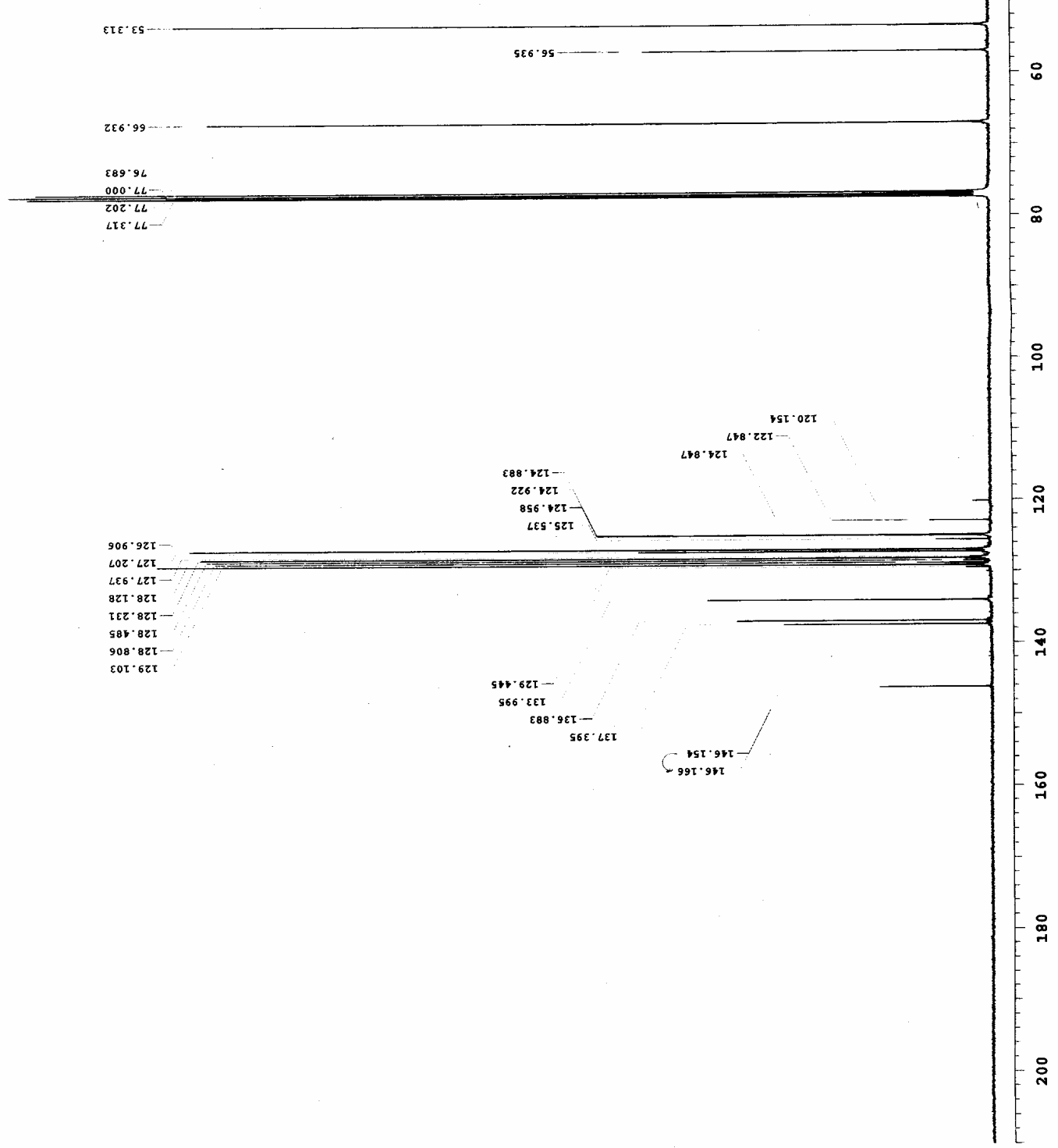


${ }^{1} \mathrm{H}$ NMR $\left(400 \mathrm{MHz}, \mathrm{CDCl}_{3}\right)$
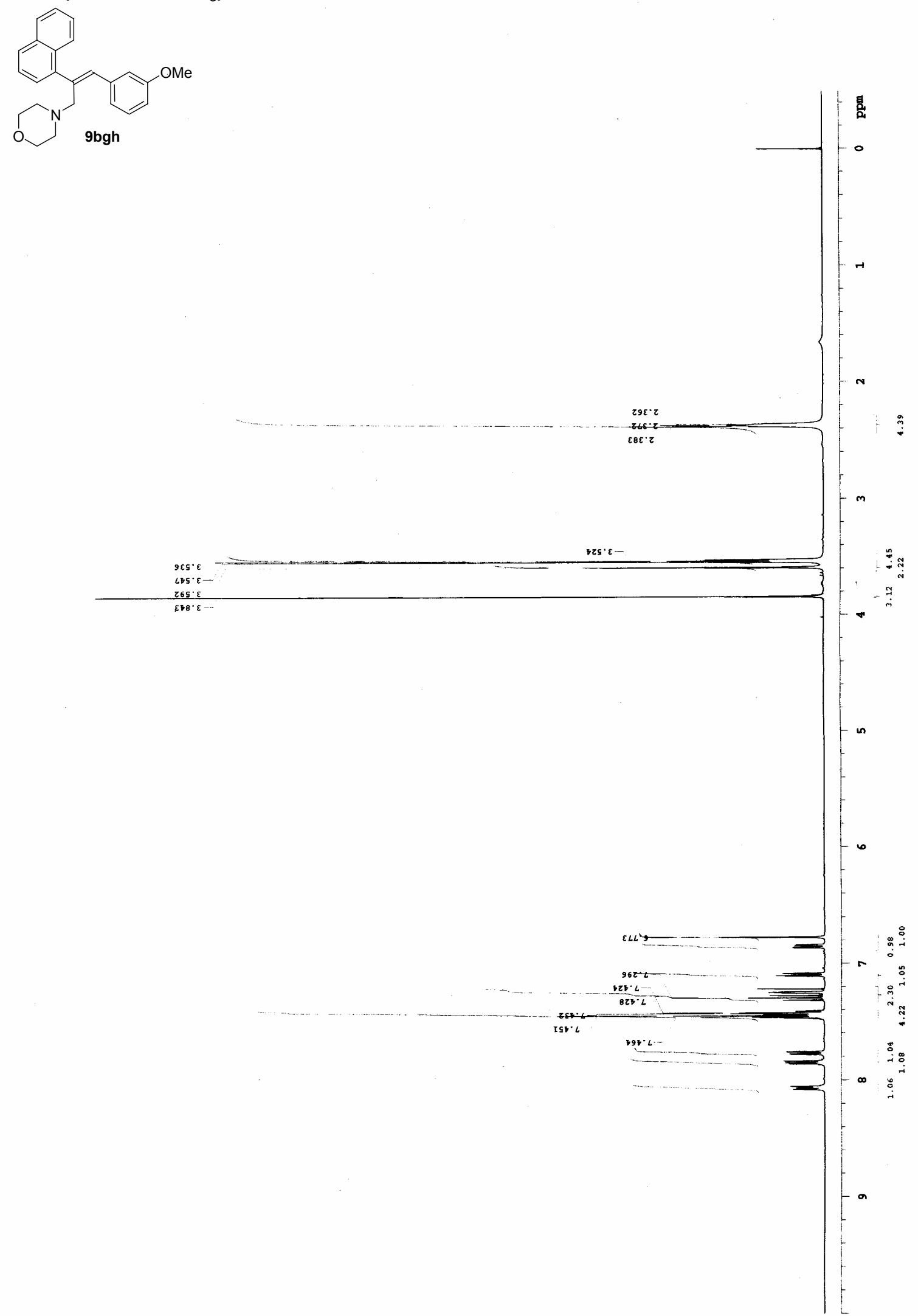
${ }^{13} \mathrm{C} \mathrm{NMR}\left(100 \mathrm{MHz}, \mathrm{CDCl}_{3}\right)$

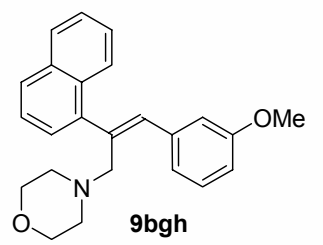

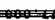

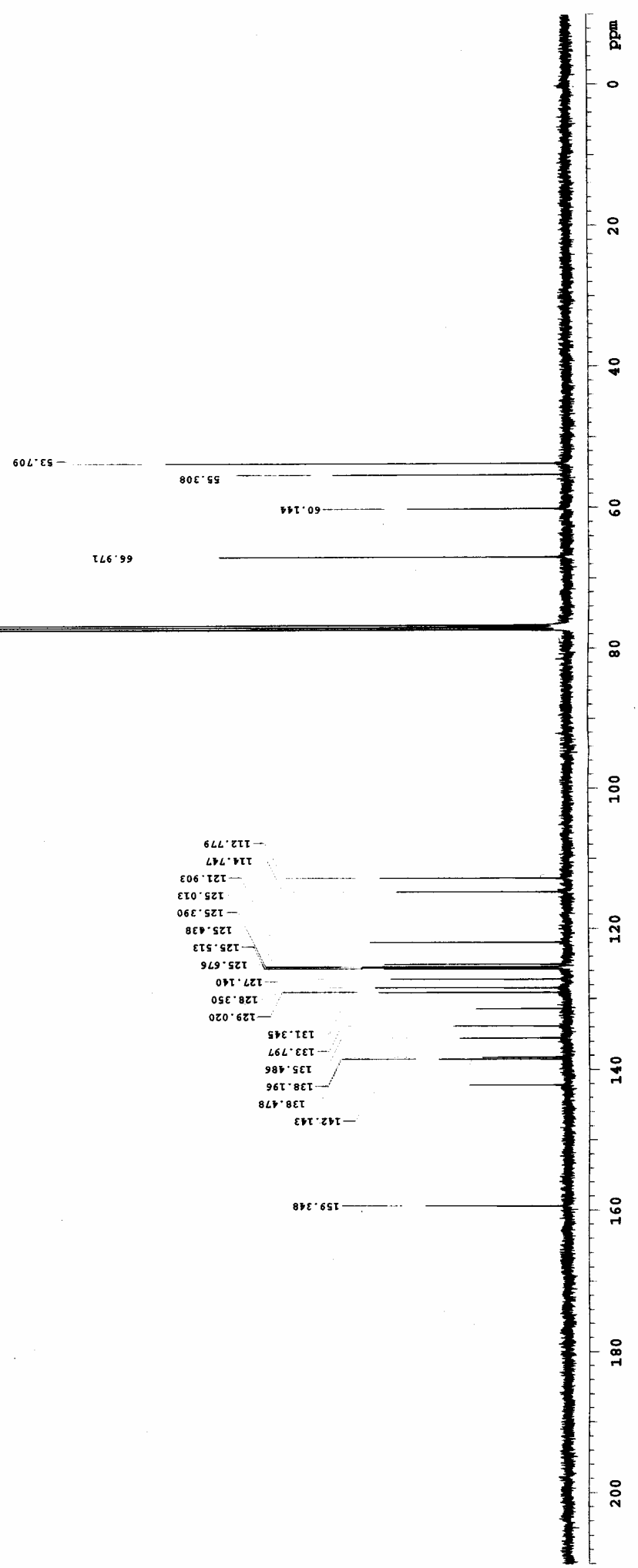


${ }^{1} \mathrm{H}$ NMR $\left(400 \mathrm{MHz}, \mathrm{CDCl}_{3}\right)$
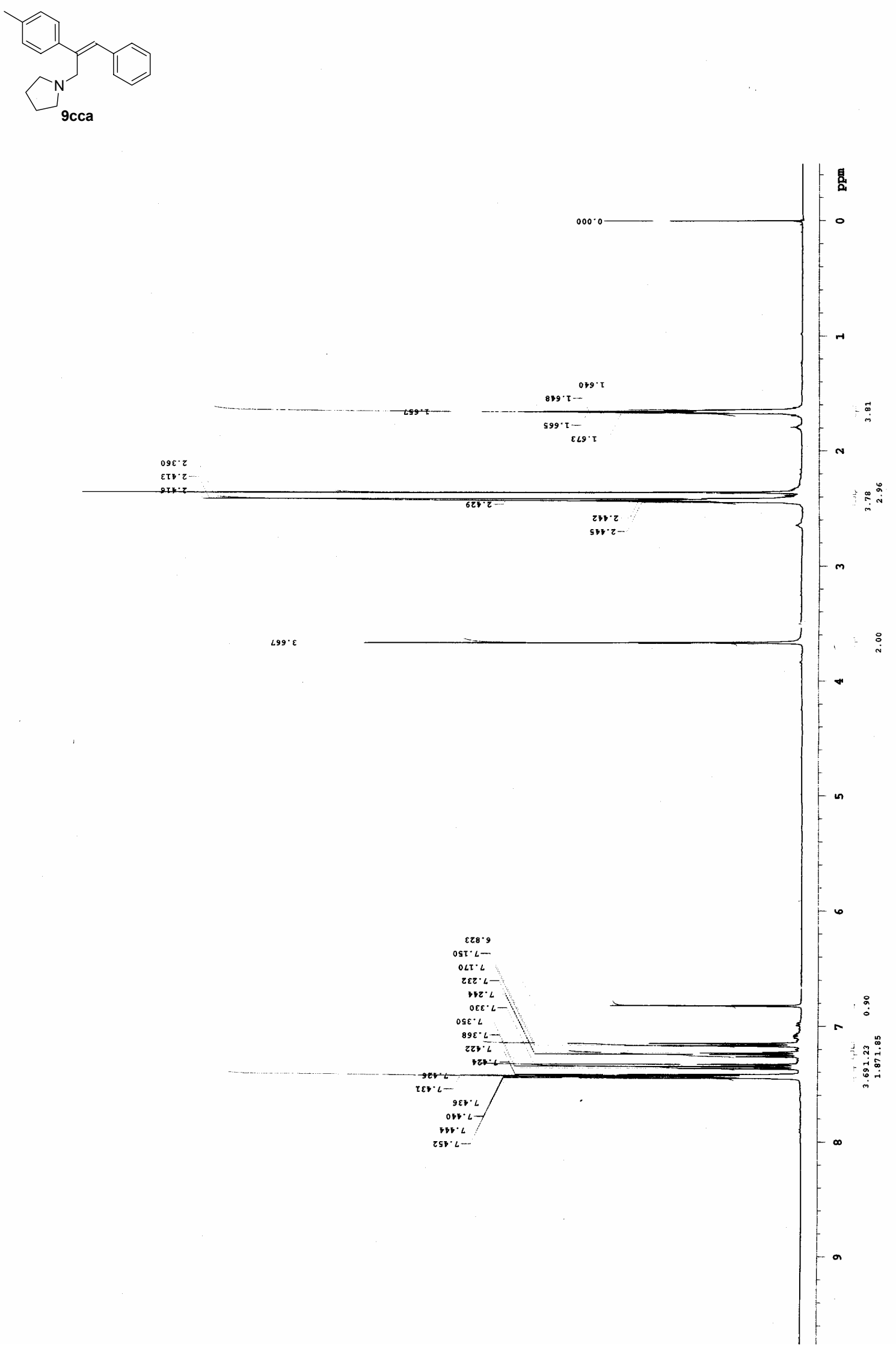
${ }^{13} \mathrm{C} \mathrm{NMR}$ (100MHz, $\mathrm{CDCl}_{3}$ )<smiles>Cc1ccc(C(=Cc2ccccc2)CN2CCCC2)cc1</smiles>

tos $98 \mathrm{~T}$ -

$002 \cdot 9 \mathrm{st}$

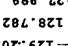

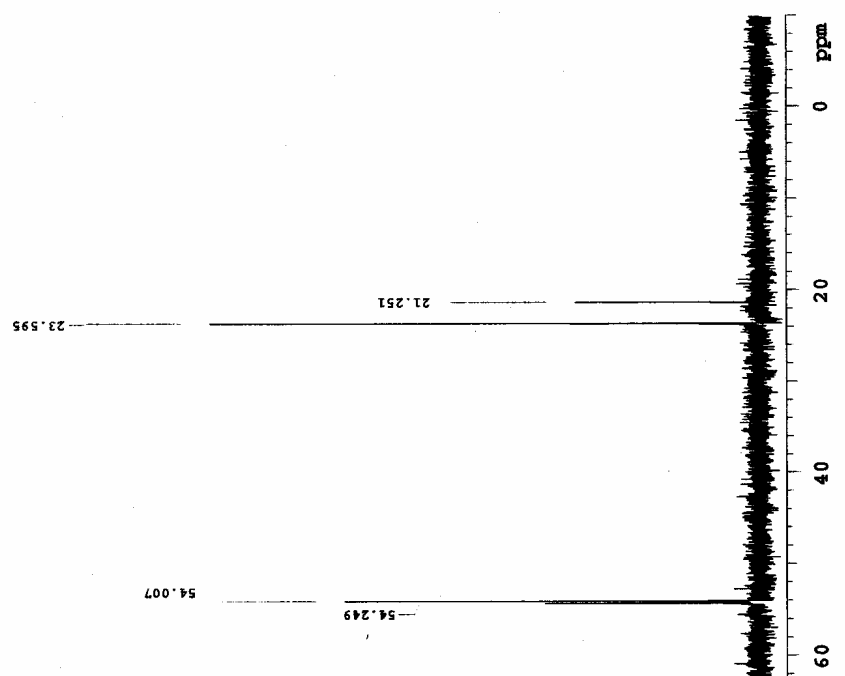

289.9

zะน' $9 \varepsilon \tau-$

$80 L \cdot \operatorname{L\tau T}$

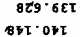


${ }^{1} \mathrm{H}$ NMR (400MHz, $\mathrm{CDCl}_{3}$ )

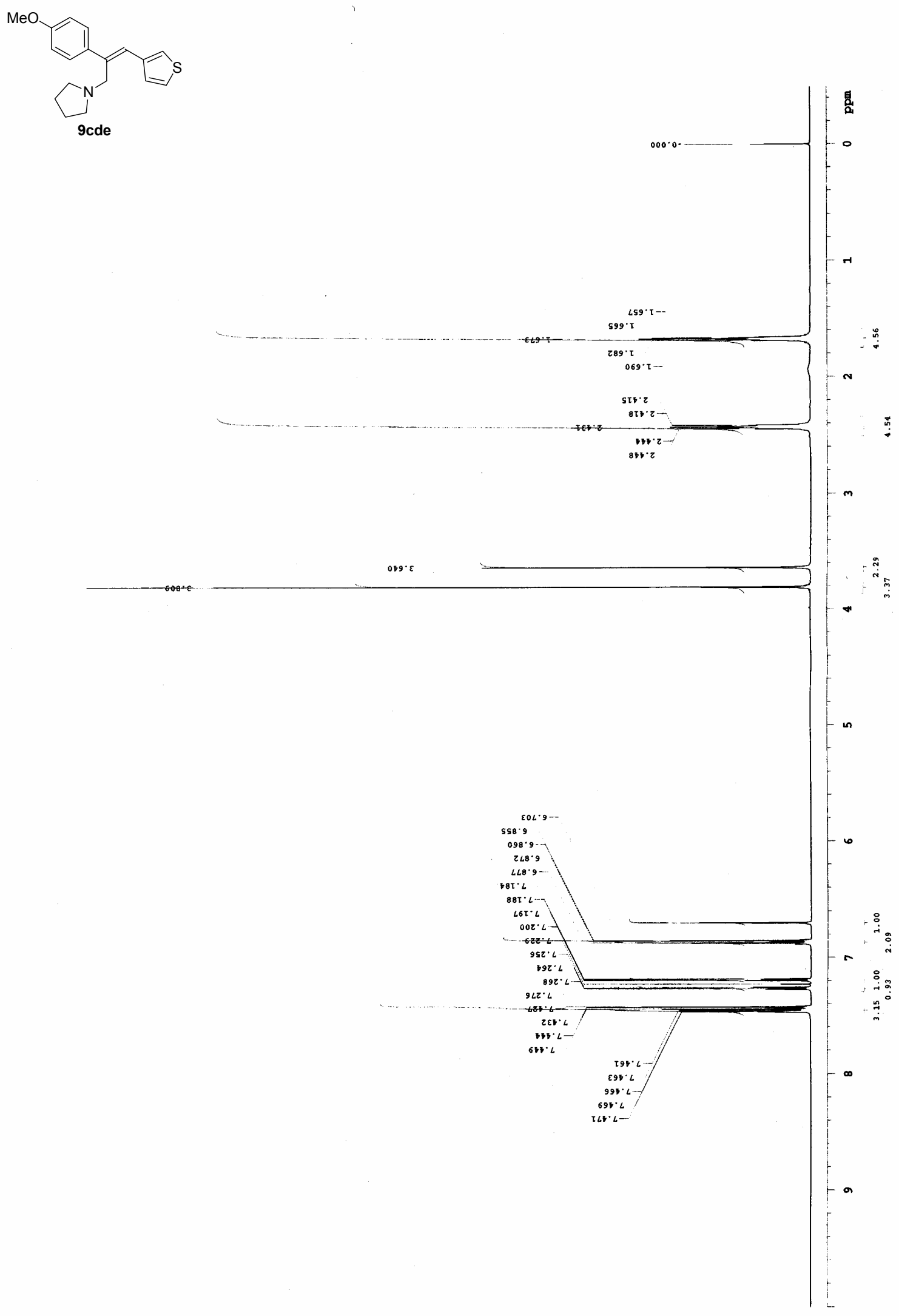


${ }^{13} \mathrm{C}$ NMR $\left(100 \mathrm{MHz}, \mathrm{CDCl}_{3}\right)$
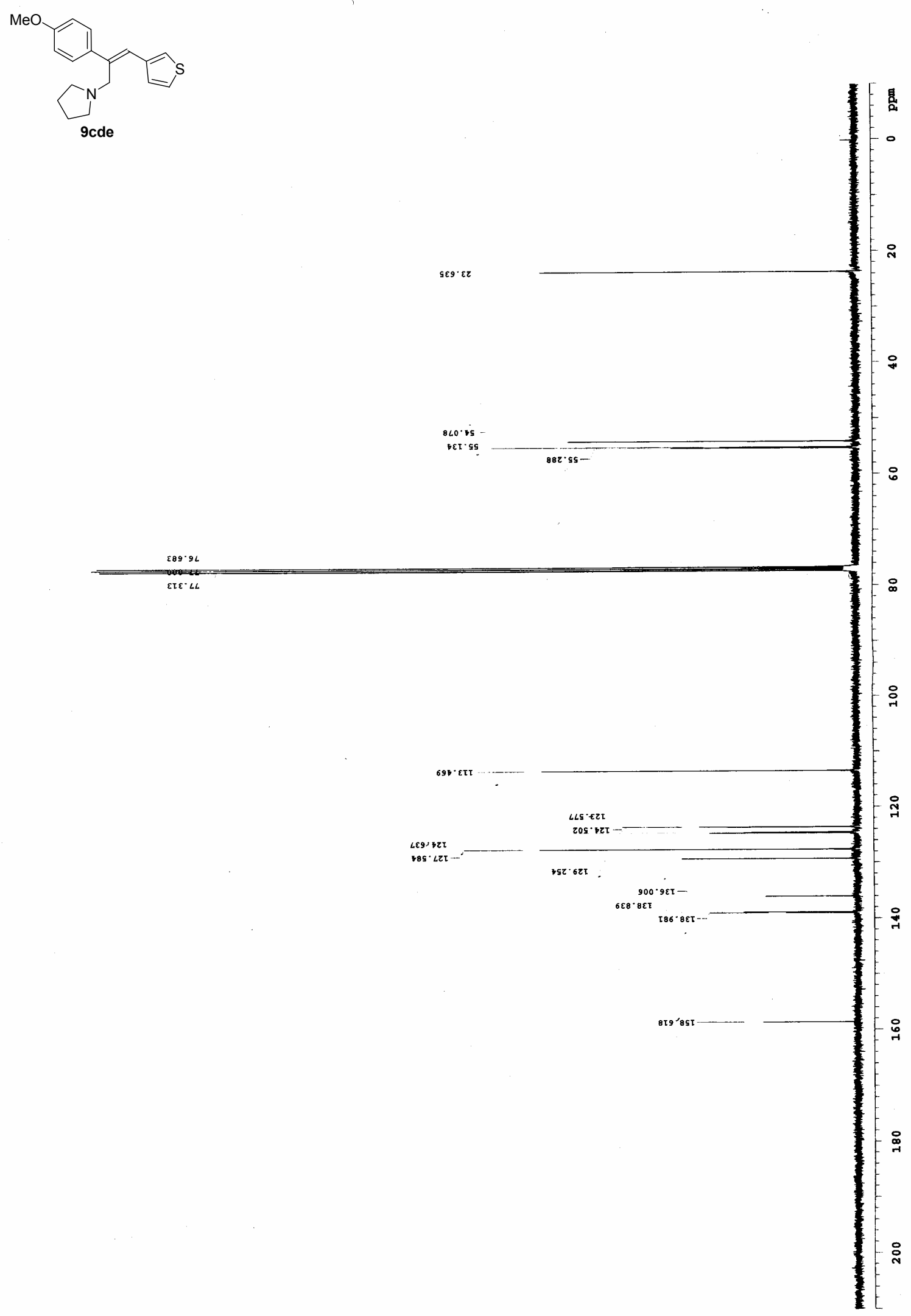
${ }^{1} \mathrm{H}$ NMR $\left(400 \mathrm{MHz}, \mathrm{CDCl}_{3}\right)$

$\mathrm{EtO}_{2} \mathrm{C}$<smiles>Cc1ccccc1C=C(CN1CCCC1)c1ccccc1</smiles>

9cij

$\llcorner\llcorner\mathcal{\varepsilon} \cdot \tau-$

ฤ6E. 1

2 รโจ.

$9 \angle S^{\circ}$

$58 S^{\circ} \tau$
$\varepsilon 65^{\circ} \tau$
$z 09^{\circ} \tau$
$0 . g^{\circ} \tau$

$0 \mathrm{Ta} \cdot \mathrm{T}$

$L L z \cdot z$
$90 \varepsilon \cdot z$

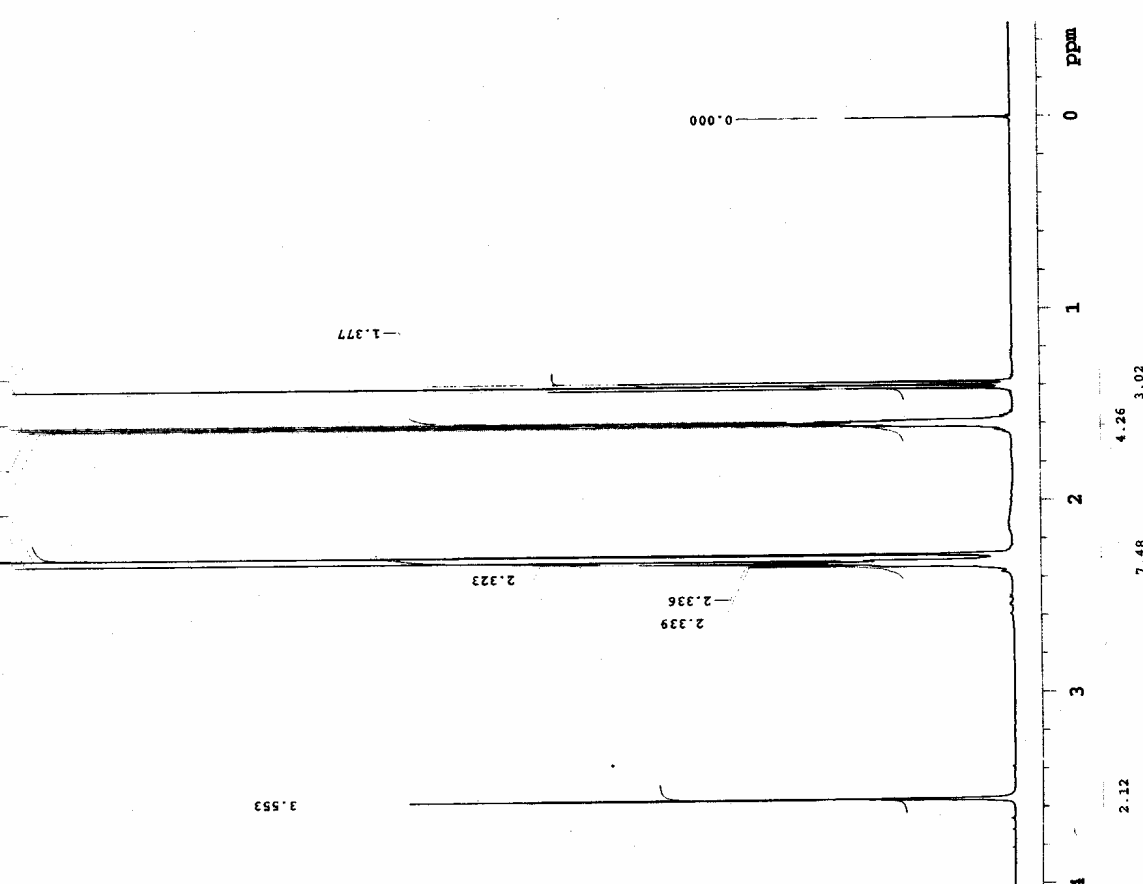

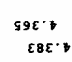

8เะ $\div-$

TOF.

$86 \mathrm{~T} \cdot \mathrm{L}$

$00 z \cdot\llcorner$
$z \varepsilon z \cdot L$

$z \varepsilon \tau \cdot L-$
$\bar{s} \tau \cdot\llcorner$

$582 \cdot L$
$56 z \cdot L-$
$\varepsilon 0 \varepsilon \cdot L$

Eor. $\angle$

हค9. $L$

t8ז $\cdot 2$

$\angle B T \cdot L$

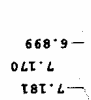

ovg. 4

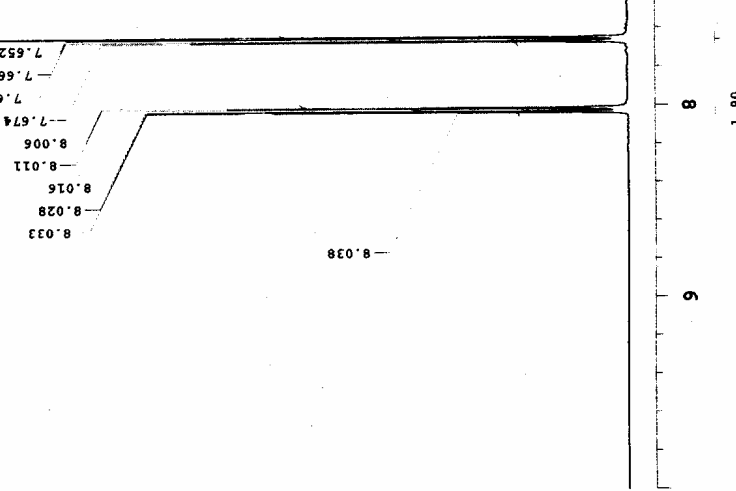

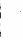

$\stackrel{\leftrightarrow}{\sim}$

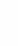


${ }^{13} \mathrm{C}$ NMR (100MHz, $\left.\mathrm{CDCl}_{3}\right)$
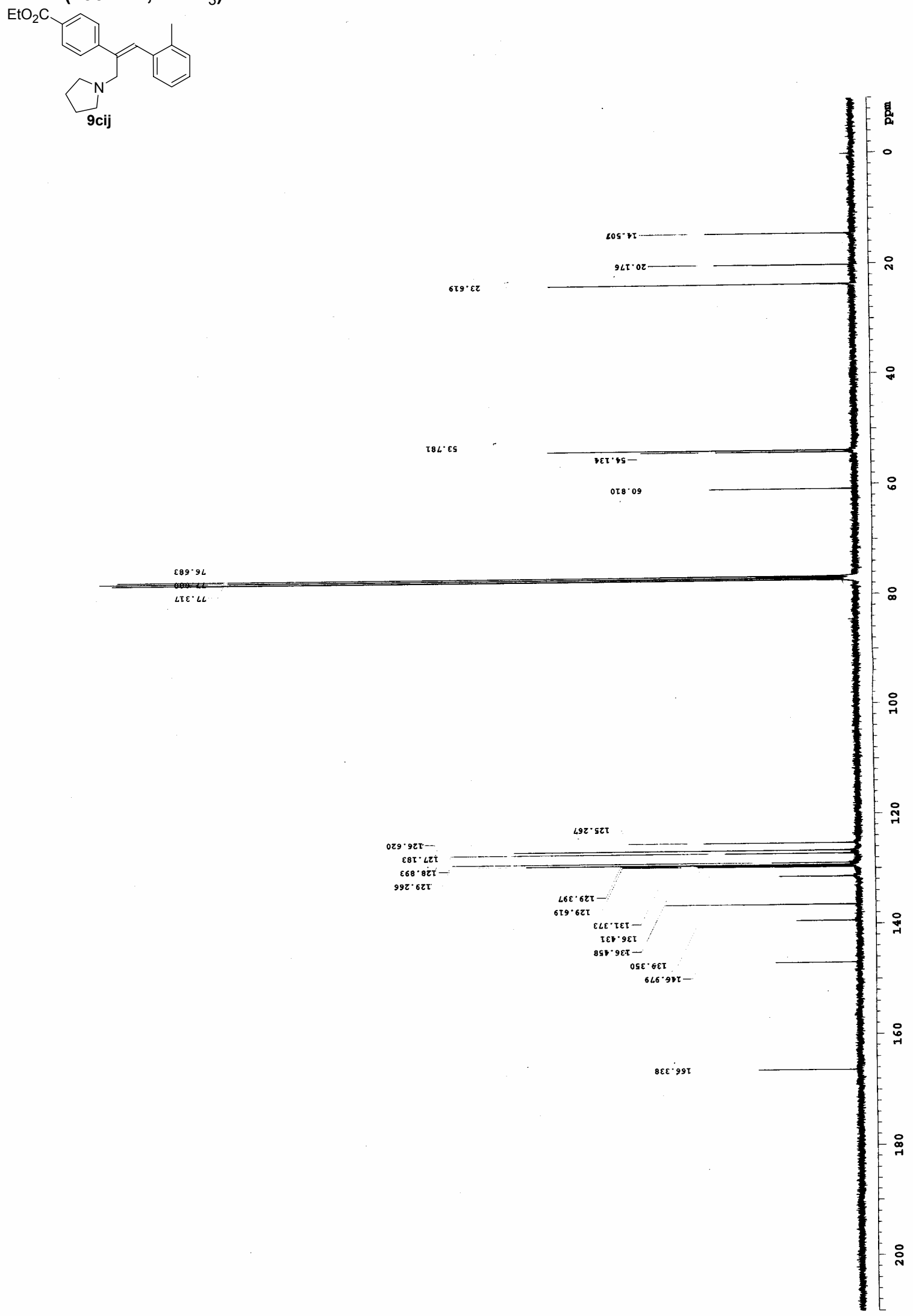

S43 
${ }^{1} \mathrm{H}$ NMR $\left(400 \mathrm{MHz}, \mathrm{CDCl}_{3}\right)$
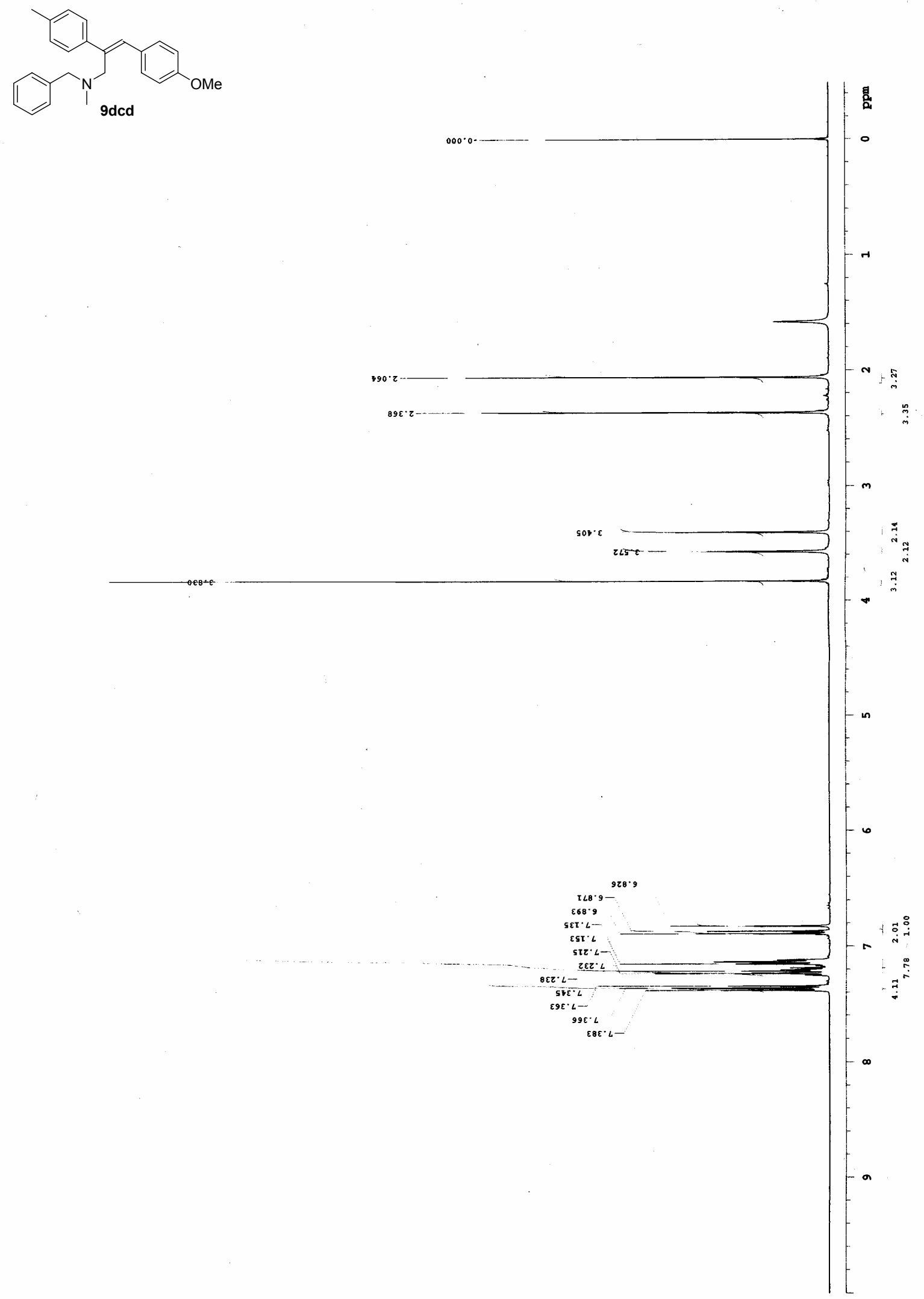
${ }^{13} \mathrm{C} \mathrm{NMR}\left(100 \mathrm{MHz}, \mathrm{CDCl}_{3}\right)$

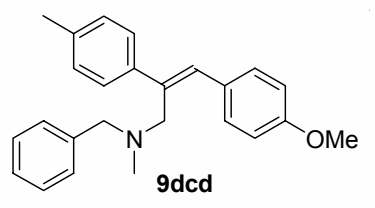

289.96

$\frac{000 \cdot \angle L}{202 \cdot L L}$

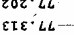

:

हZง - $8 z \tau$

$896^{\circ} \mathrm{Bz}$

EโE. OET

$9 L \cdot 0 \varepsilon$

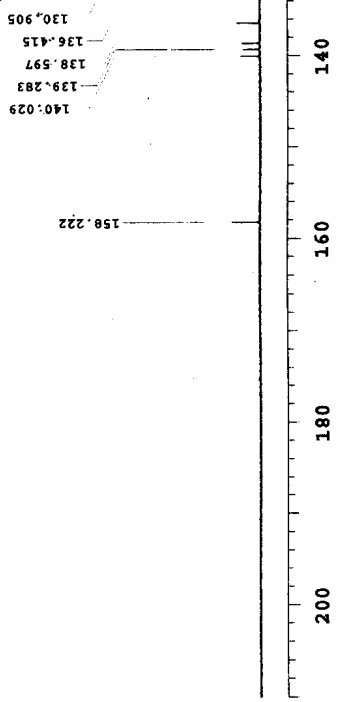

: 
${ }^{1} \mathrm{H}$ NMR $\left(400 \mathrm{MHz}, \mathrm{CDCl}_{3}\right)$
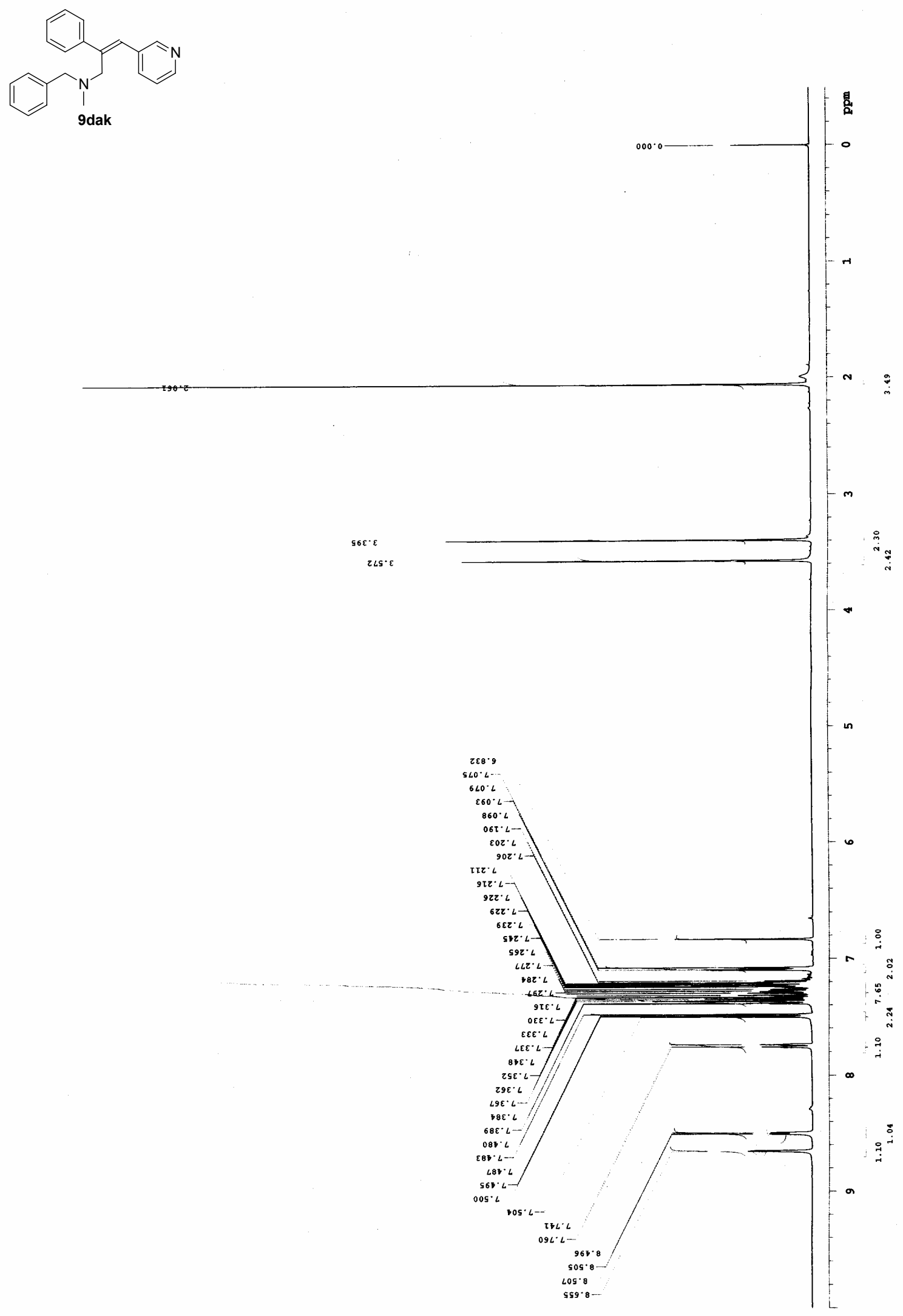
${ }^{13} \mathrm{C} \mathrm{NMR}\left(100 \mathrm{MHz}, \mathrm{CDCl}_{3}\right)$

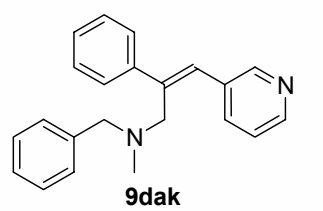

$\varepsilon 89 \cdot 94$

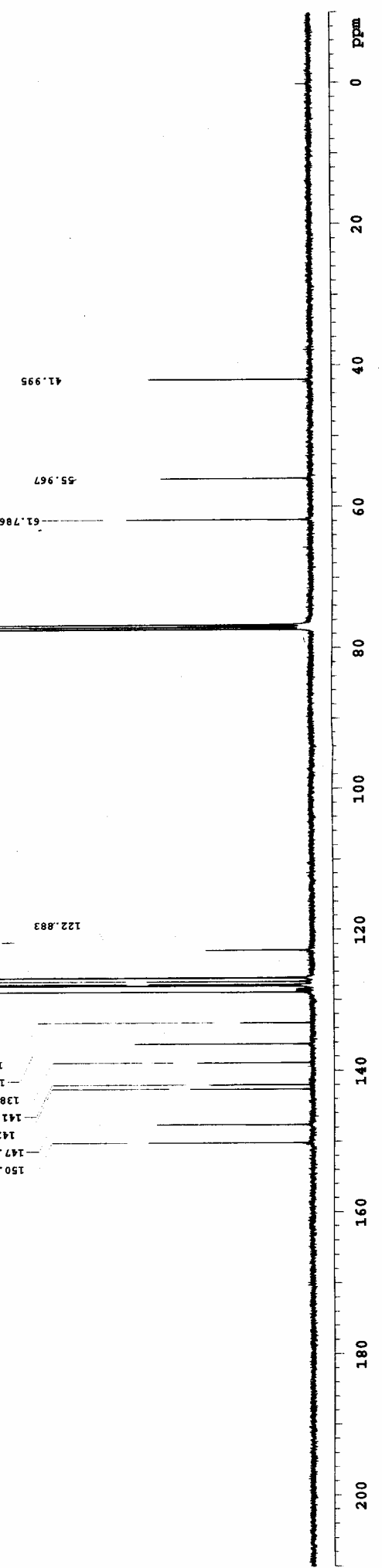


${ }^{1} \mathrm{H}$ NMR $\left(400 \mathrm{MHz}, \mathrm{CDCl}_{3}\right)$<smiles>CCOC(=O)c1ccc(/C=C(\CN(C)Cc2ccccc2)c2ccsc2)cc1</smiles>
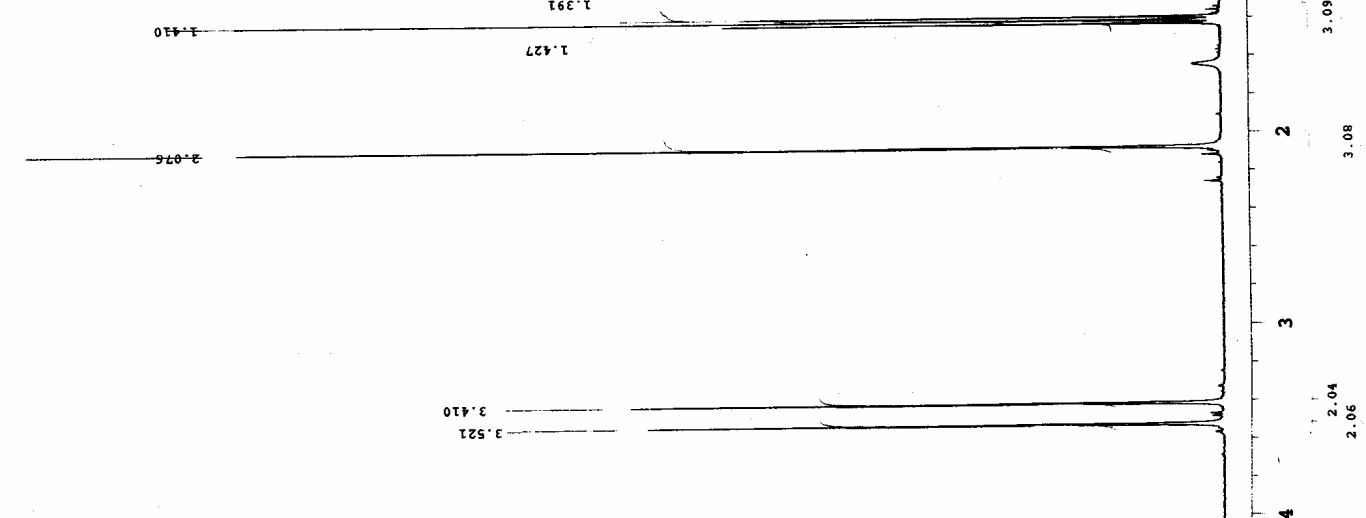

$\angle L E \cdot$

$09 \varepsilon^{\circ} \cdot$

เกเ

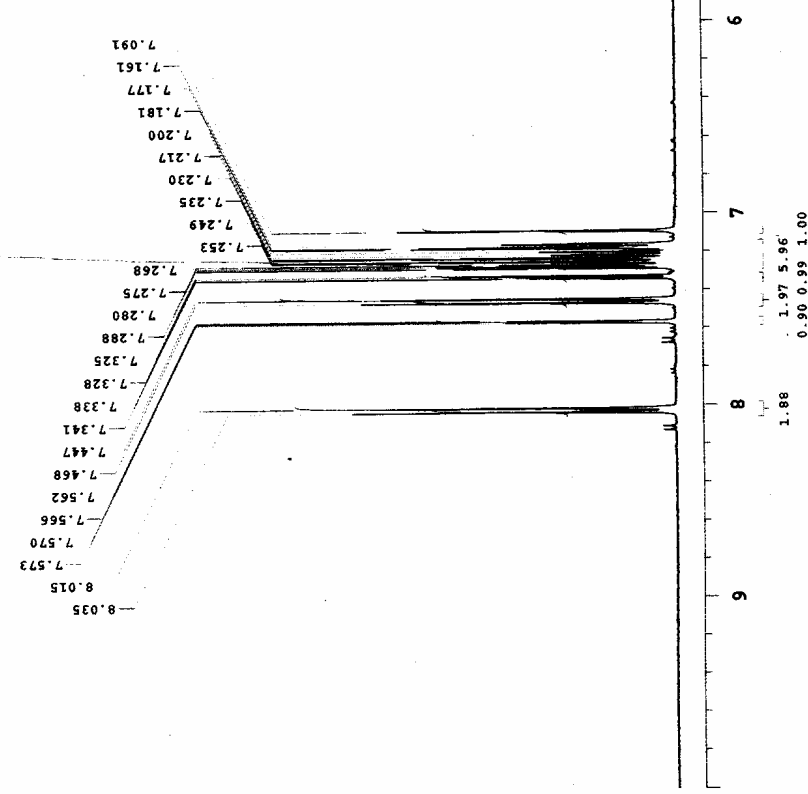


${ }^{13} \mathrm{C} \mathrm{NMR}$ (100MHz, $\mathrm{CDCl}_{3}$ )
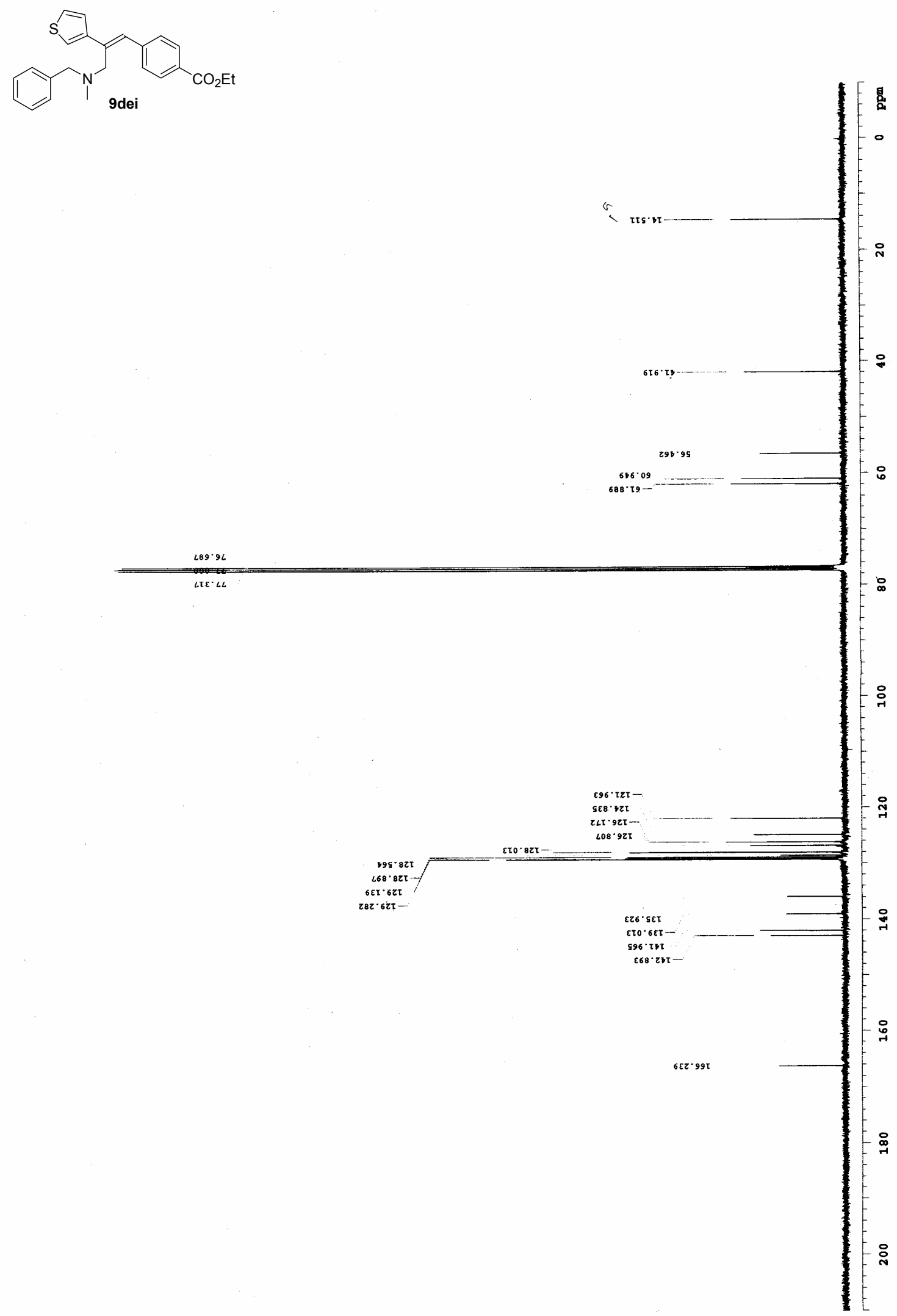
${ }^{1} \mathrm{H}$ NMR $\left(400 \mathrm{MHz}, \mathrm{CDCl}_{3}\right)$<smiles>CN(CC(=Cc1ccc(Cl)cc1)c1ccc(C#N)cc1)Cc1ccccc1</smiles>

9dlm

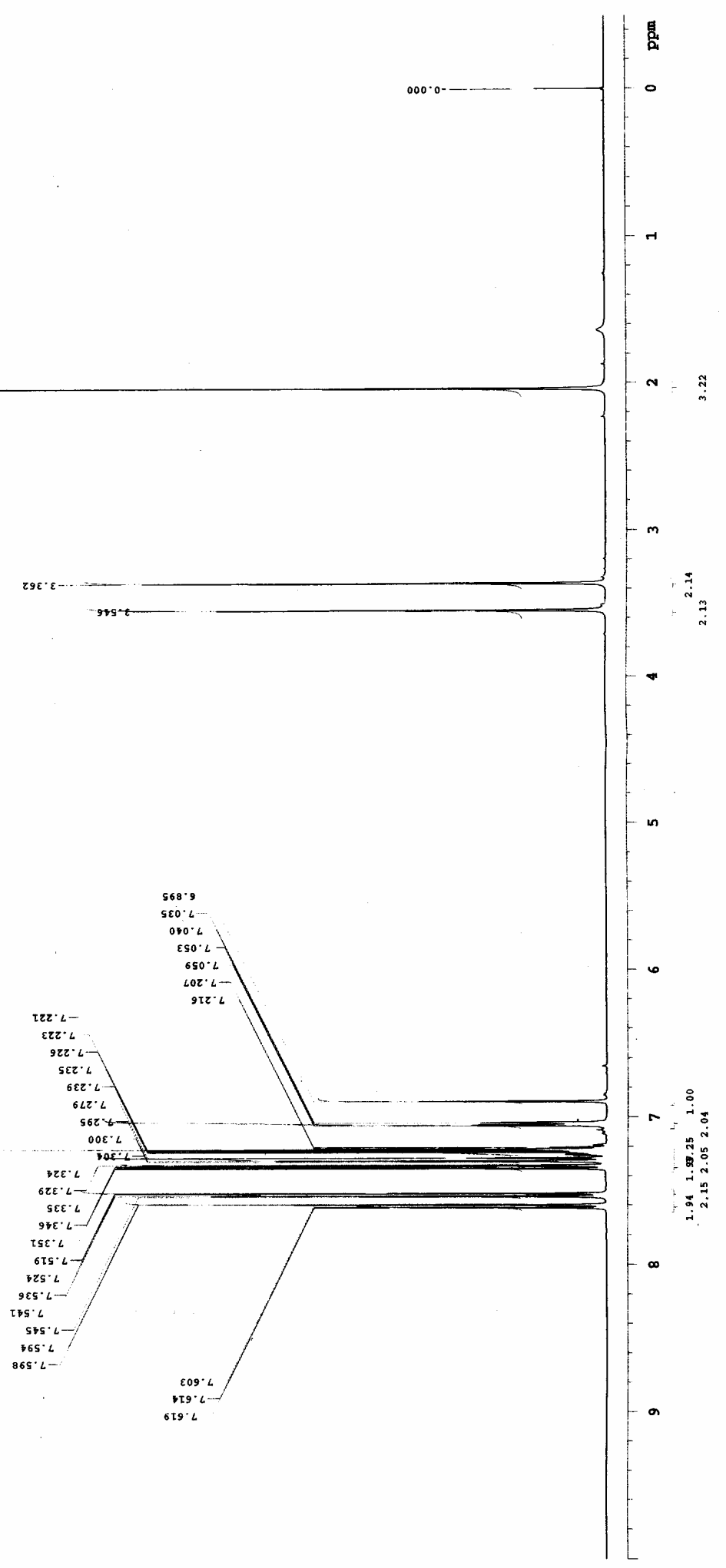


${ }^{13} \mathrm{C} \mathrm{NMR}$ (100MHz, $\mathrm{CDCl}_{3}$ )<smiles>C[C@H](Cc1ccccc1)CN(CC(=Cc1ccc(Cl)cc1)c1ccc(C#N)cc1)Cc1ccccc1</smiles>

$268 \cdot 8 \mathrm{TI}$ -

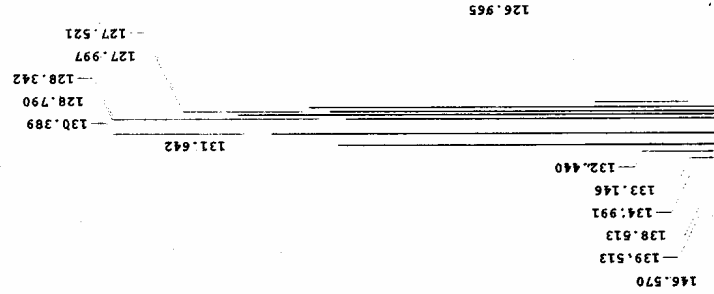

$$
0<5 \cdot 9 v
$$

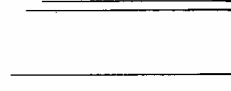


${ }^{1} \mathrm{H}$ NMR $\left(400 \mathrm{MHz}, \mathrm{CDCl}_{3}\right)$
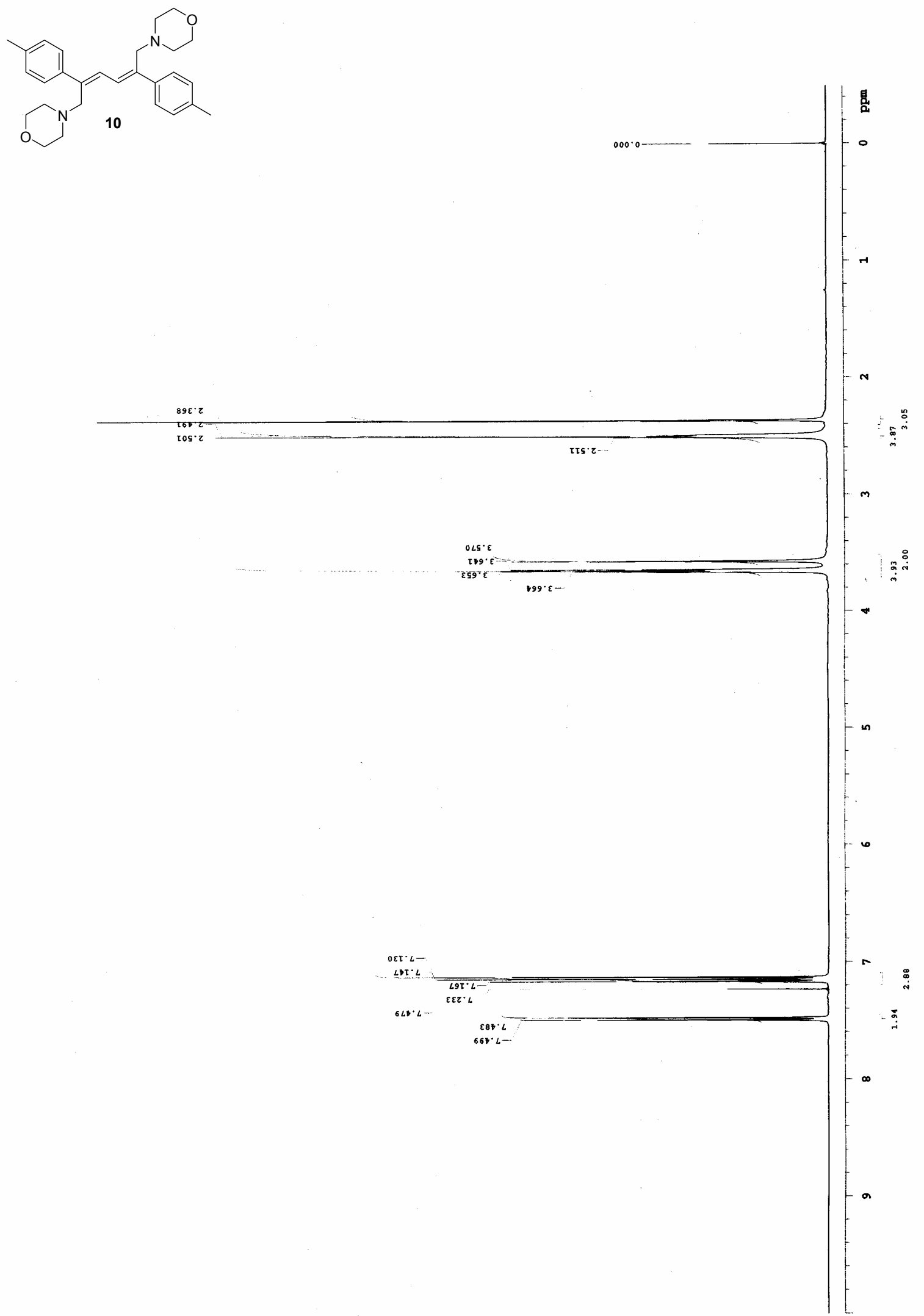
${ }^{13} \mathrm{C}$ NMR (100MHz, $\mathrm{CDCl}_{3}$ )
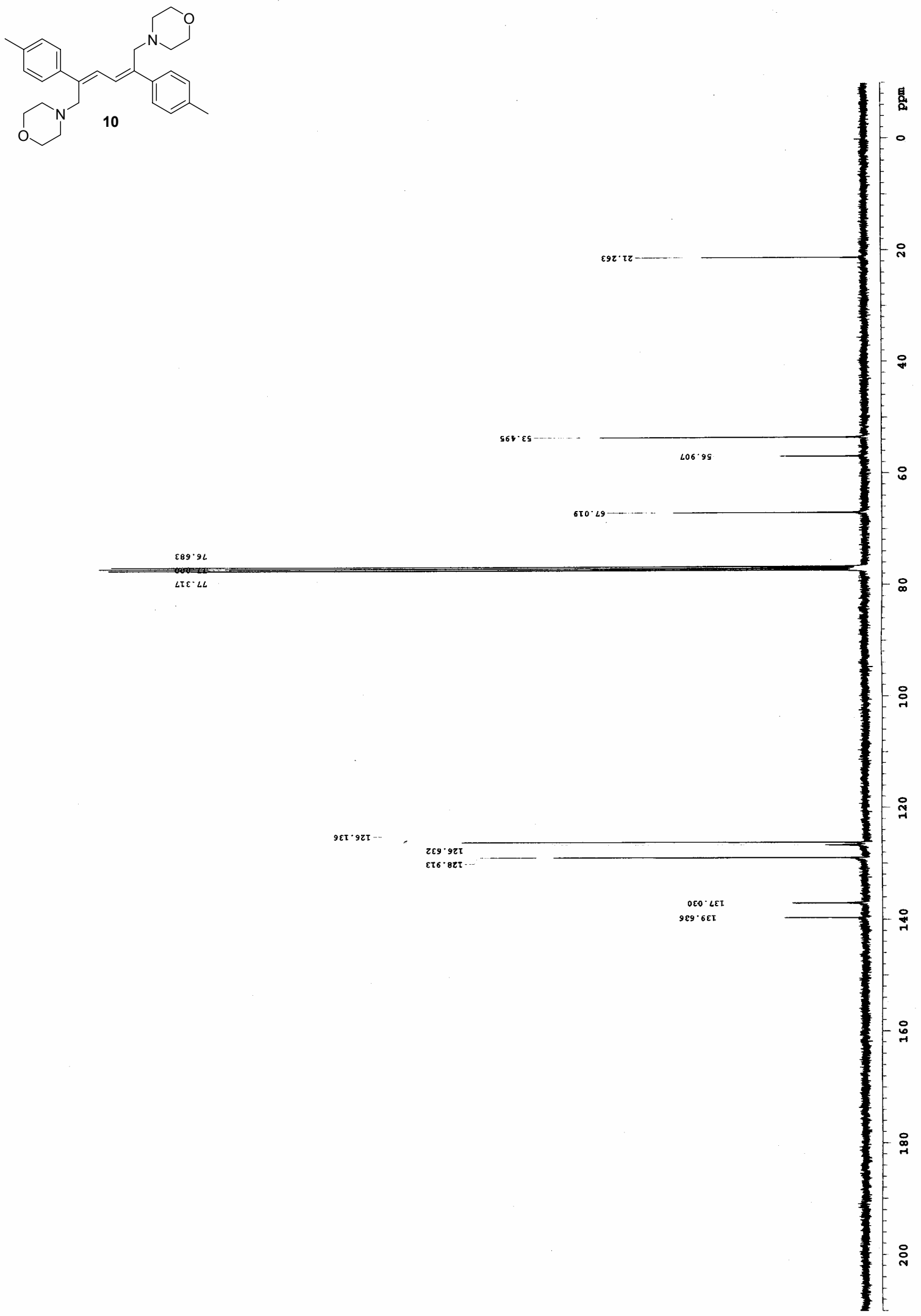
${ }^{1} \mathrm{H}$ NMR (400MHz, $\mathrm{CDCl}_{3}$ )<smiles>CCOC(=O)/C=C\C=C(\CN1CCOCC1)c1ccc(C)cc1</smiles>

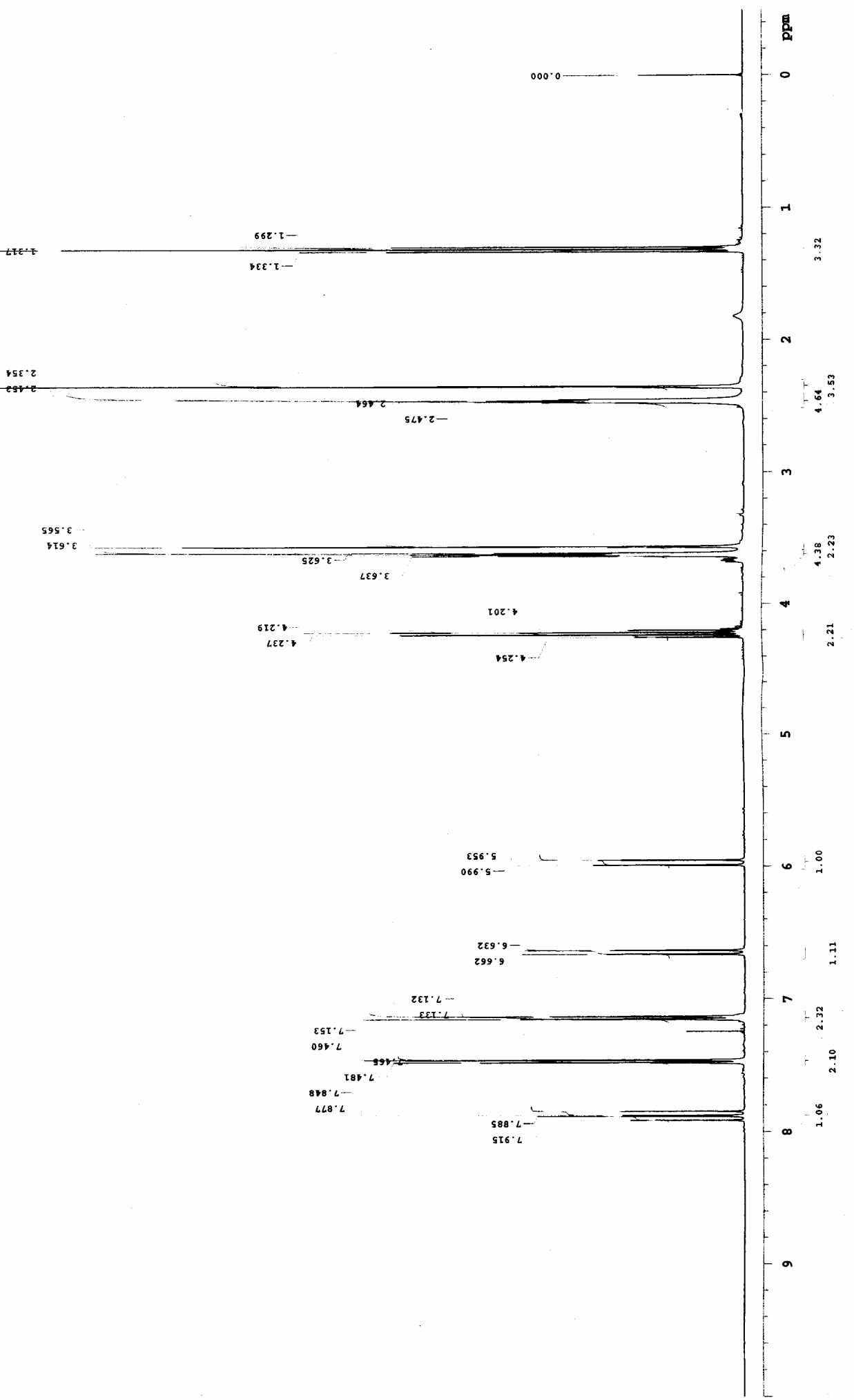


${ }^{13} \mathrm{C} \mathrm{NMR}\left(100 \mathrm{MHz}, \mathrm{CDCl}_{3}\right)$
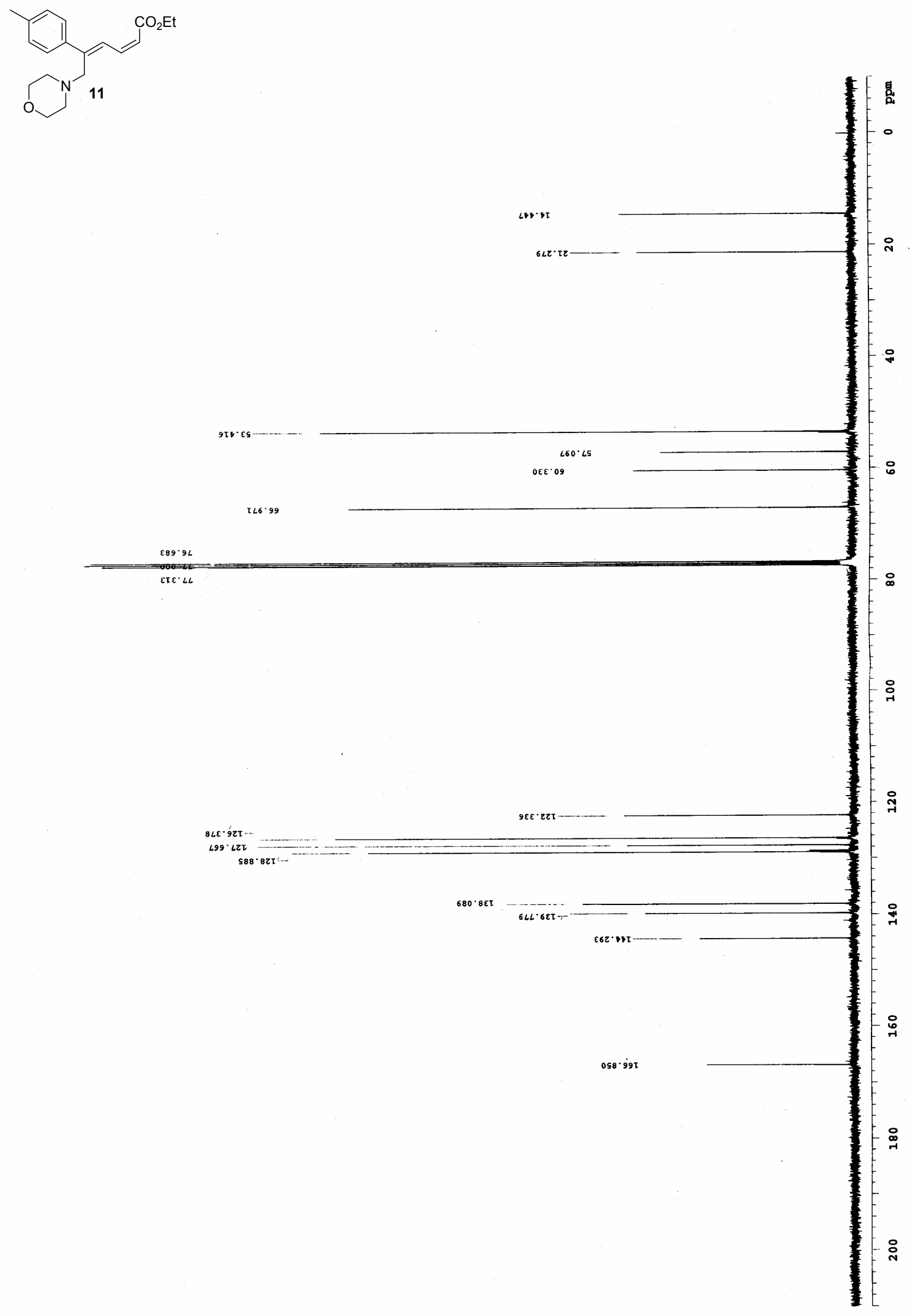
${ }^{1} \mathrm{H}$ NMR $\left(400 \mathrm{MHz}, \mathrm{CDCl}_{3}\right)$
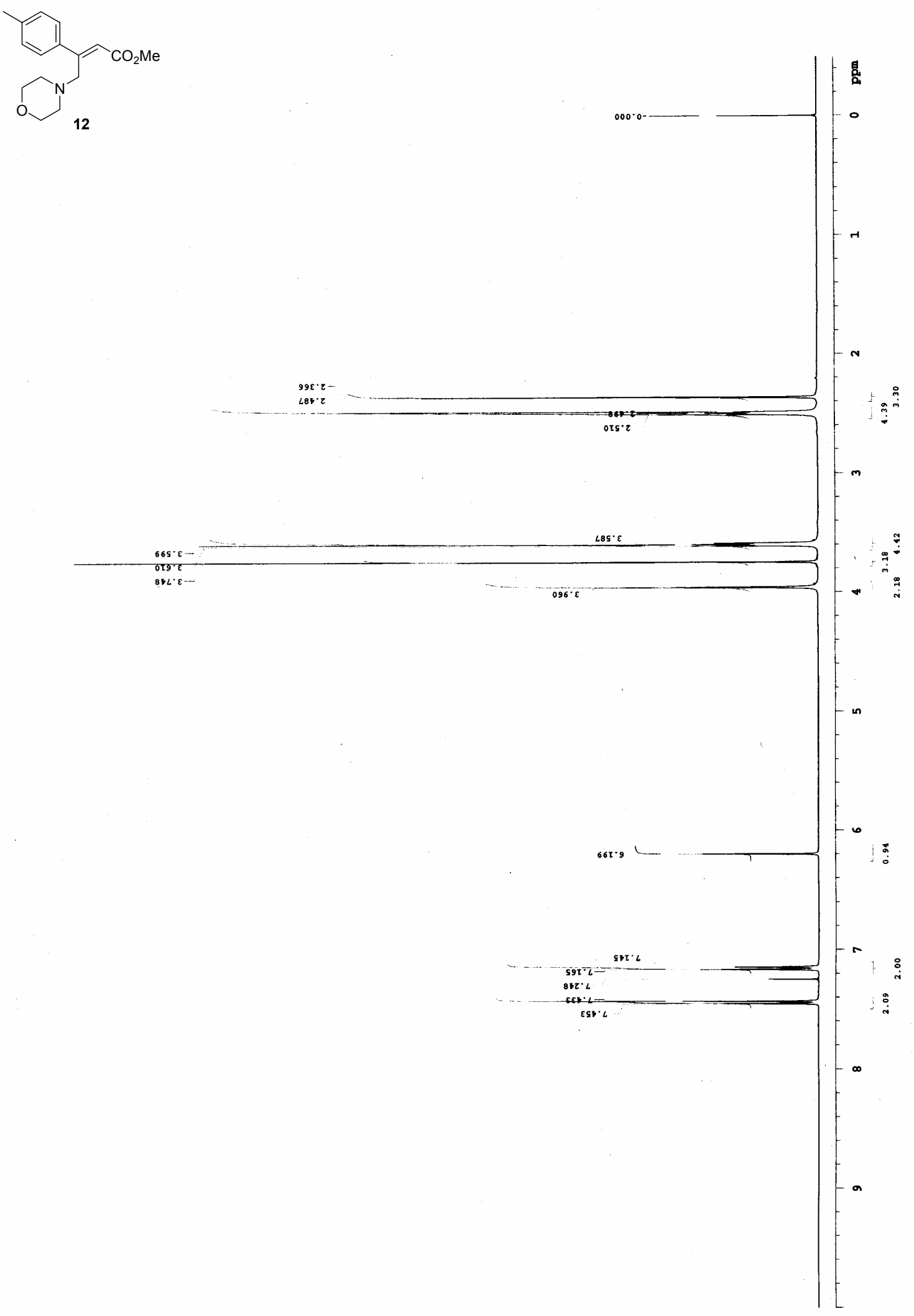
${ }^{13} \mathrm{C}$ NMR (100MHz, $\mathrm{CDCl}_{3}$ )

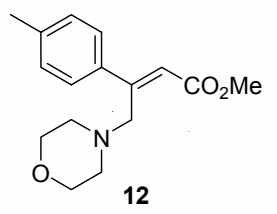

$\varepsilon 89 \cdot 96$

$20 \tau \cdot L L$

THE' T5-
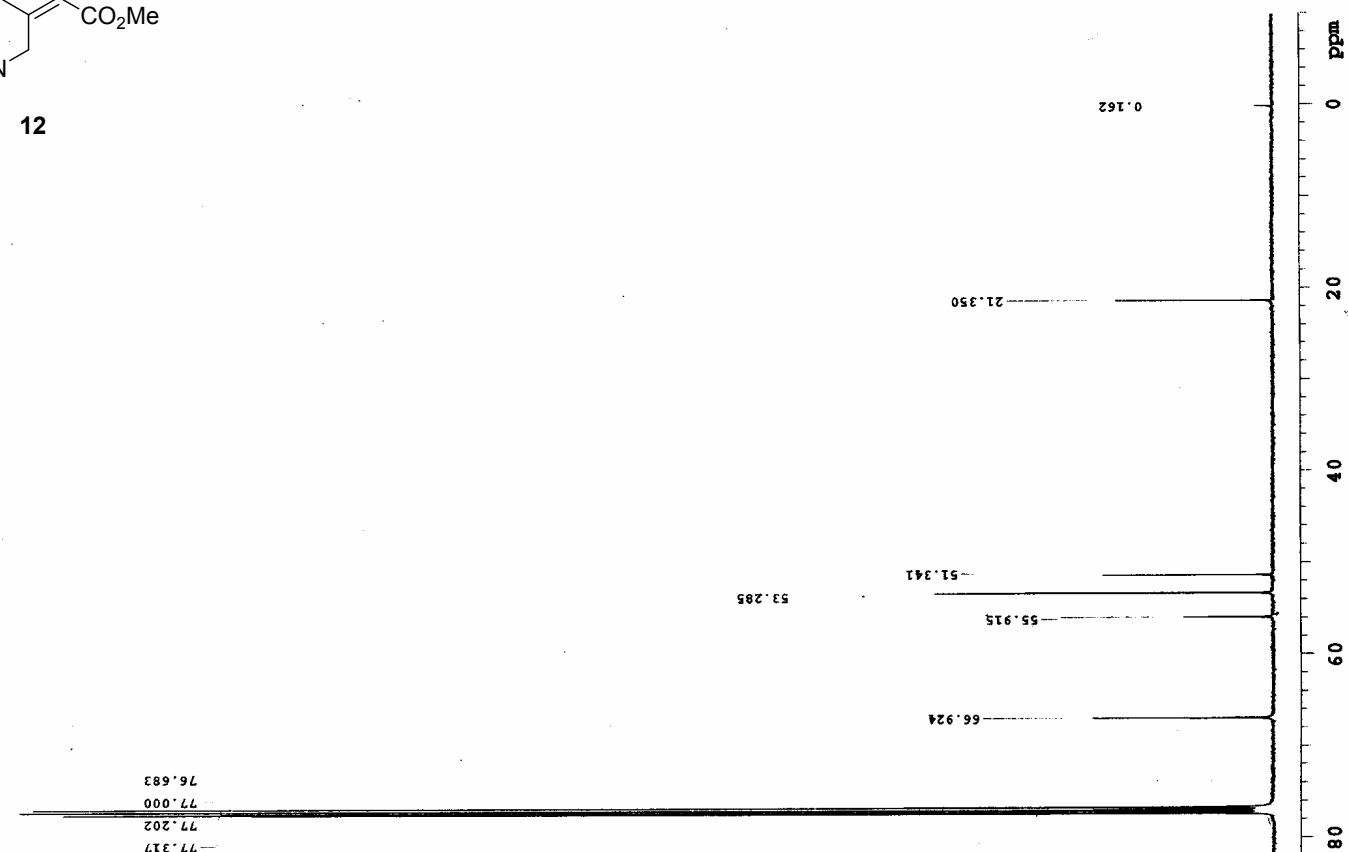
${ }^{1} \mathrm{H}$ NMR (400MHz, $\mathrm{CDCl}_{3}$ )
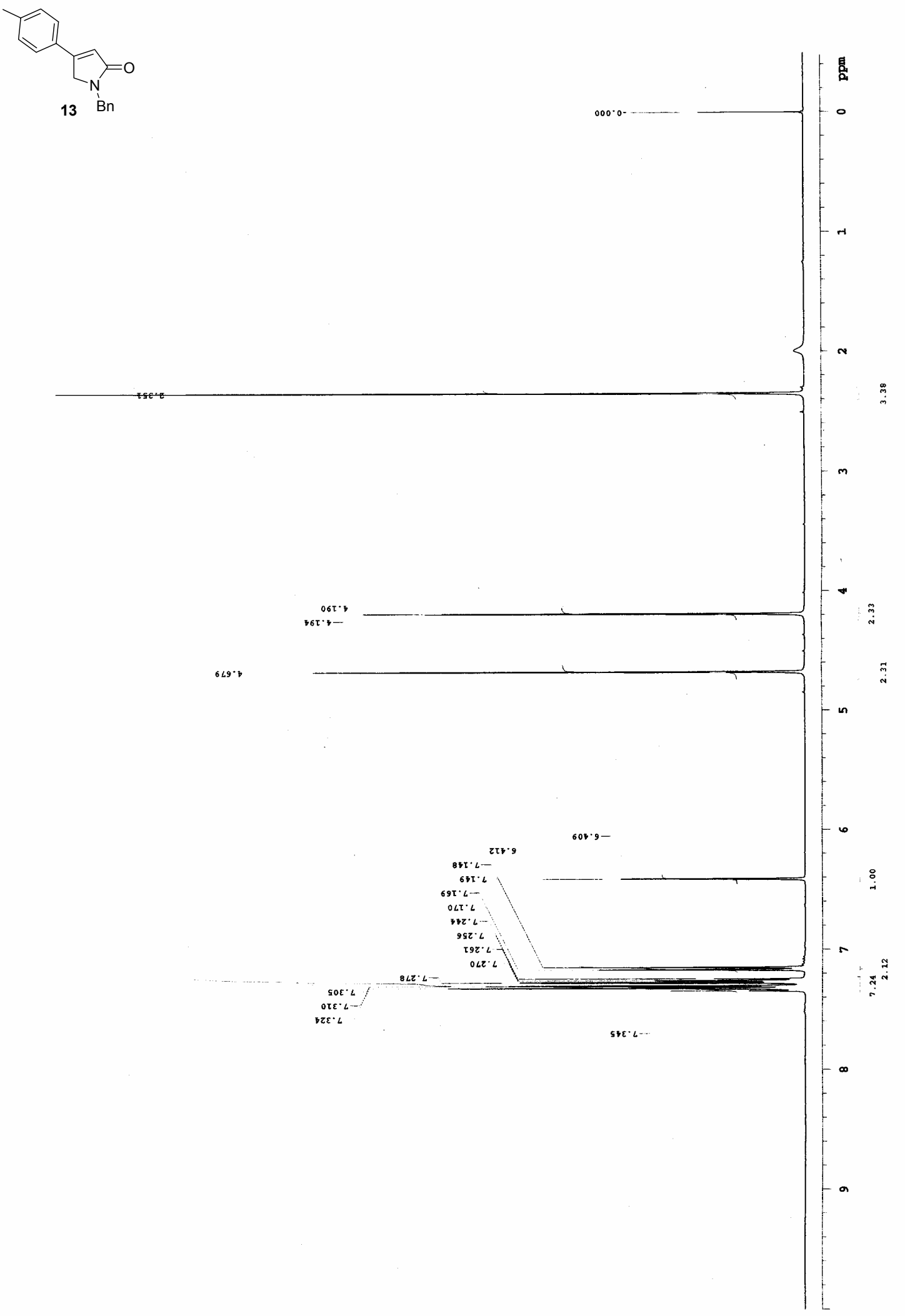
${ }^{13} \mathrm{C} \mathrm{NMR}\left(100 \mathrm{MHz}, \mathrm{CDCl}_{3}\right)$

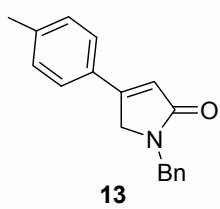

13

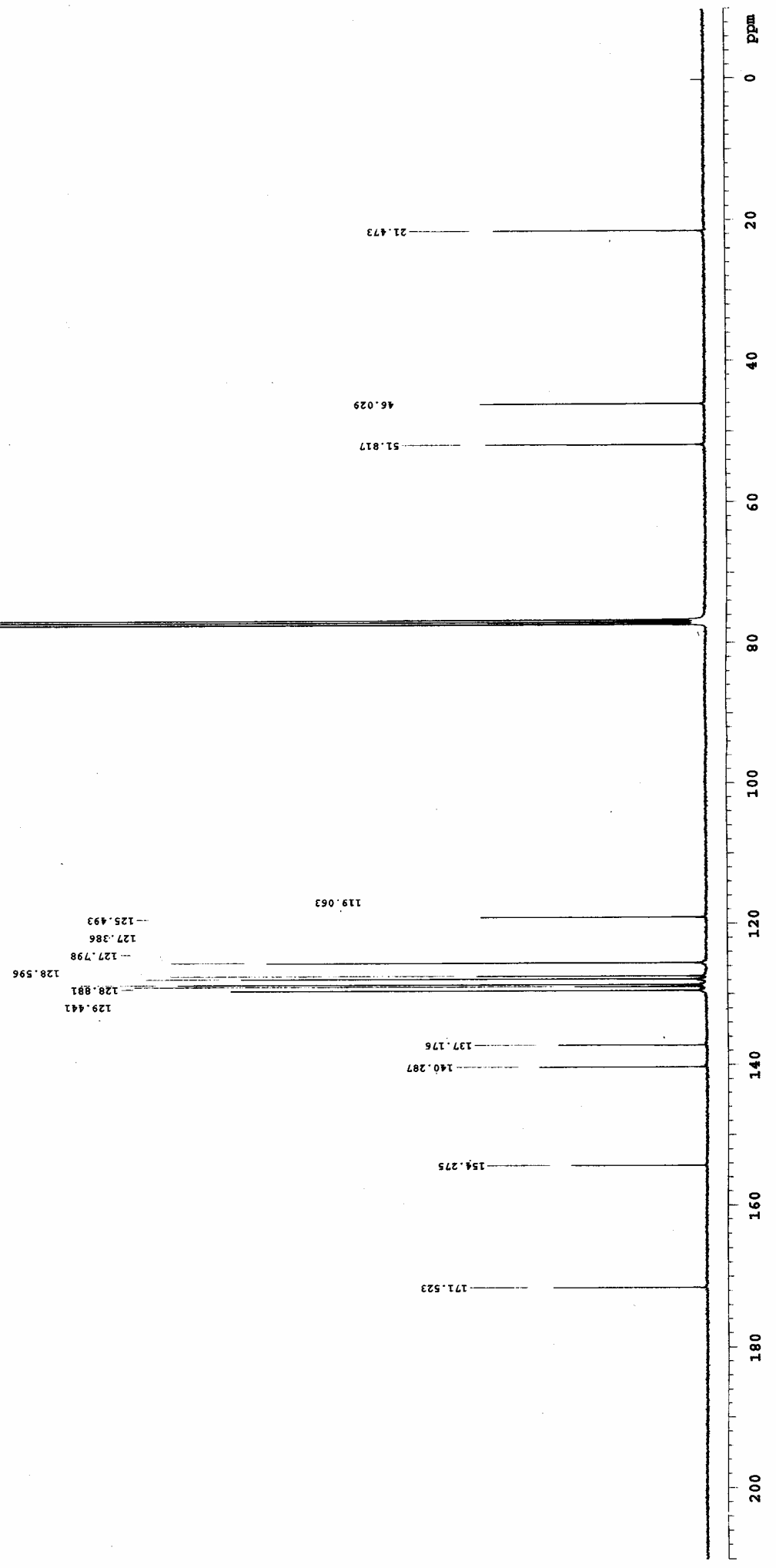


${ }^{1} \mathrm{H}$ NMR $\left(400 \mathrm{MHz}, \mathrm{CDCl}_{3}\right)$
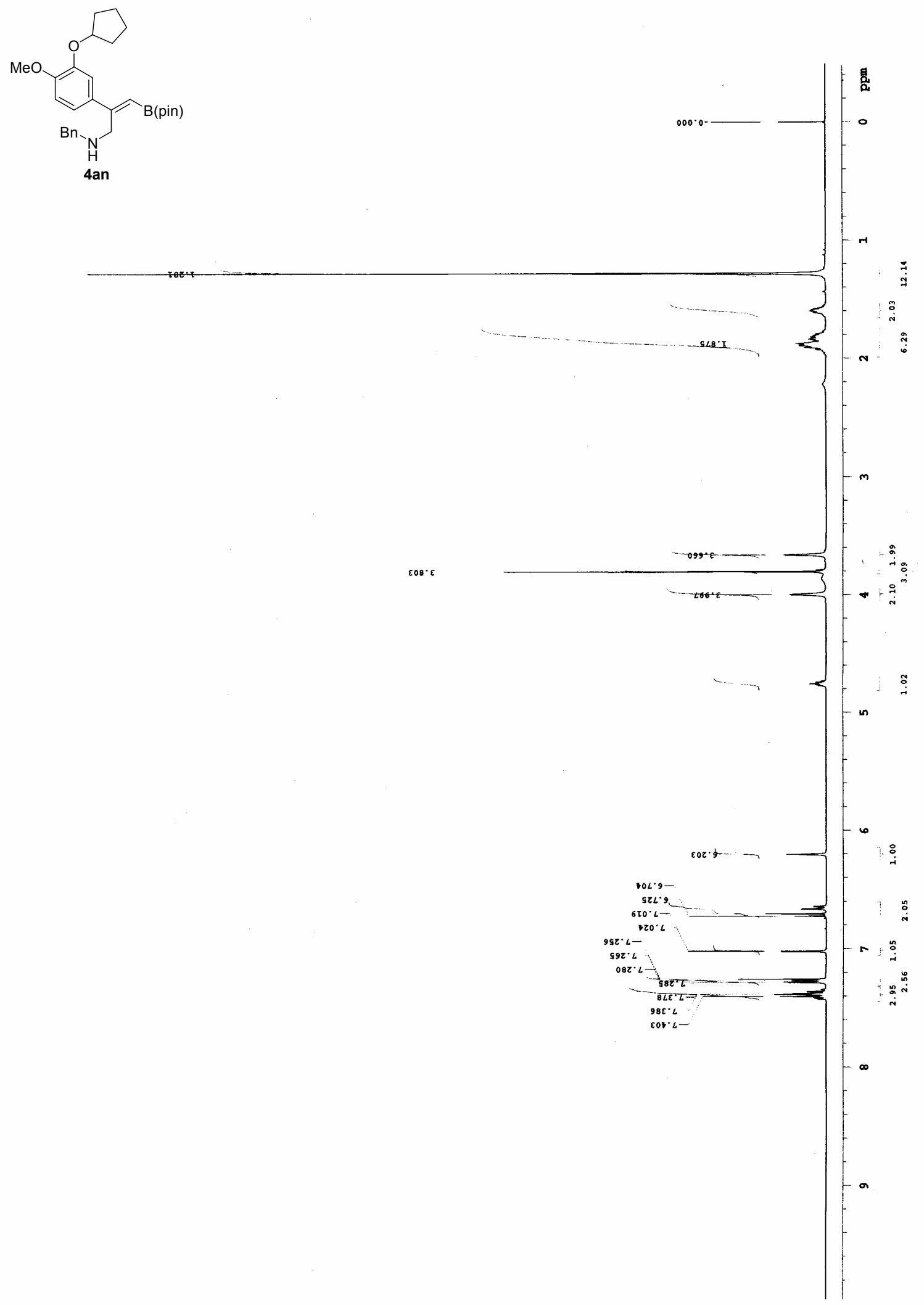
${ }^{13} \mathrm{C} \mathrm{NMR}$ (100MHz, $\mathrm{CDCl}_{3}$ )

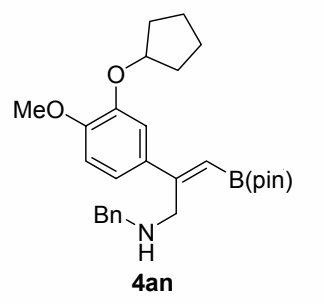

$\varepsilon 89 \cdot 9 L$
$000 \cdot \angle L$

(I)

LIE $\angle L-$

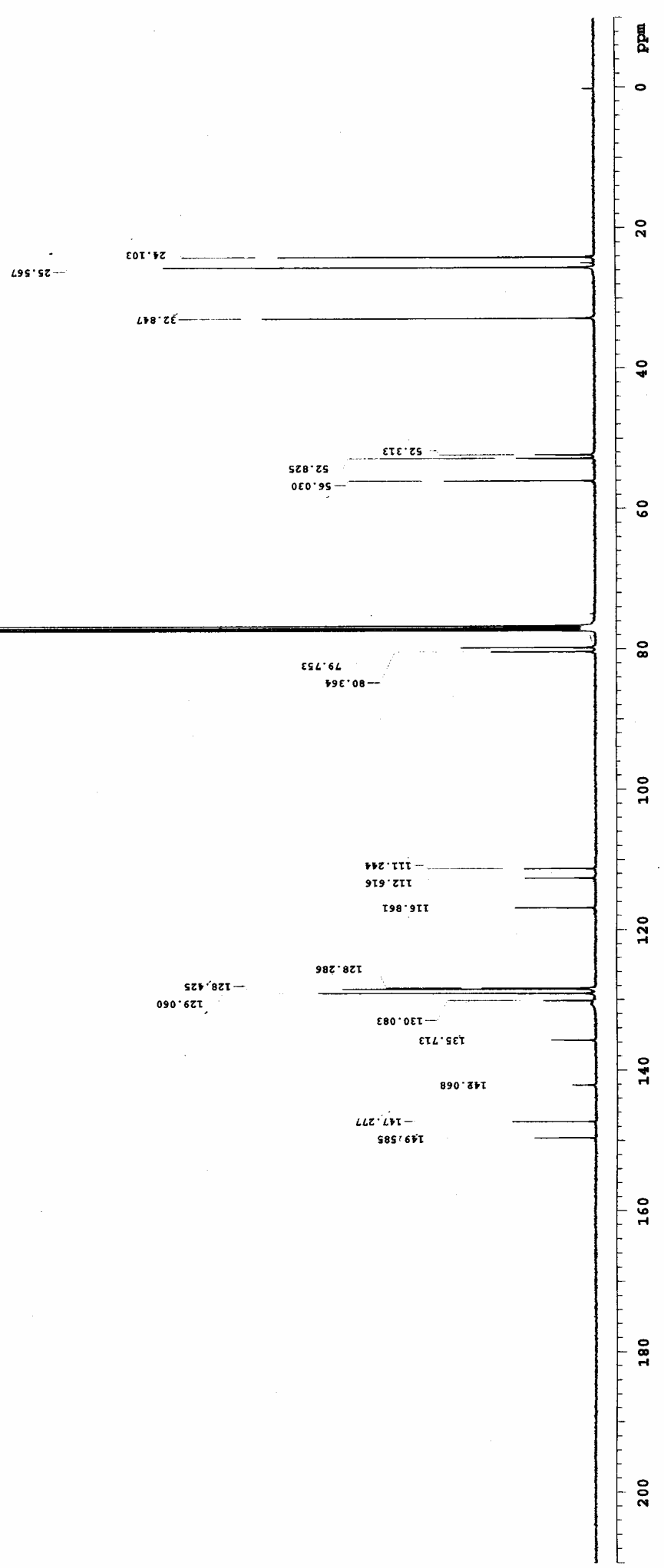


${ }^{1} \mathrm{H}$ NMR (400MHz, $\mathrm{CDCl}_{3}$ )
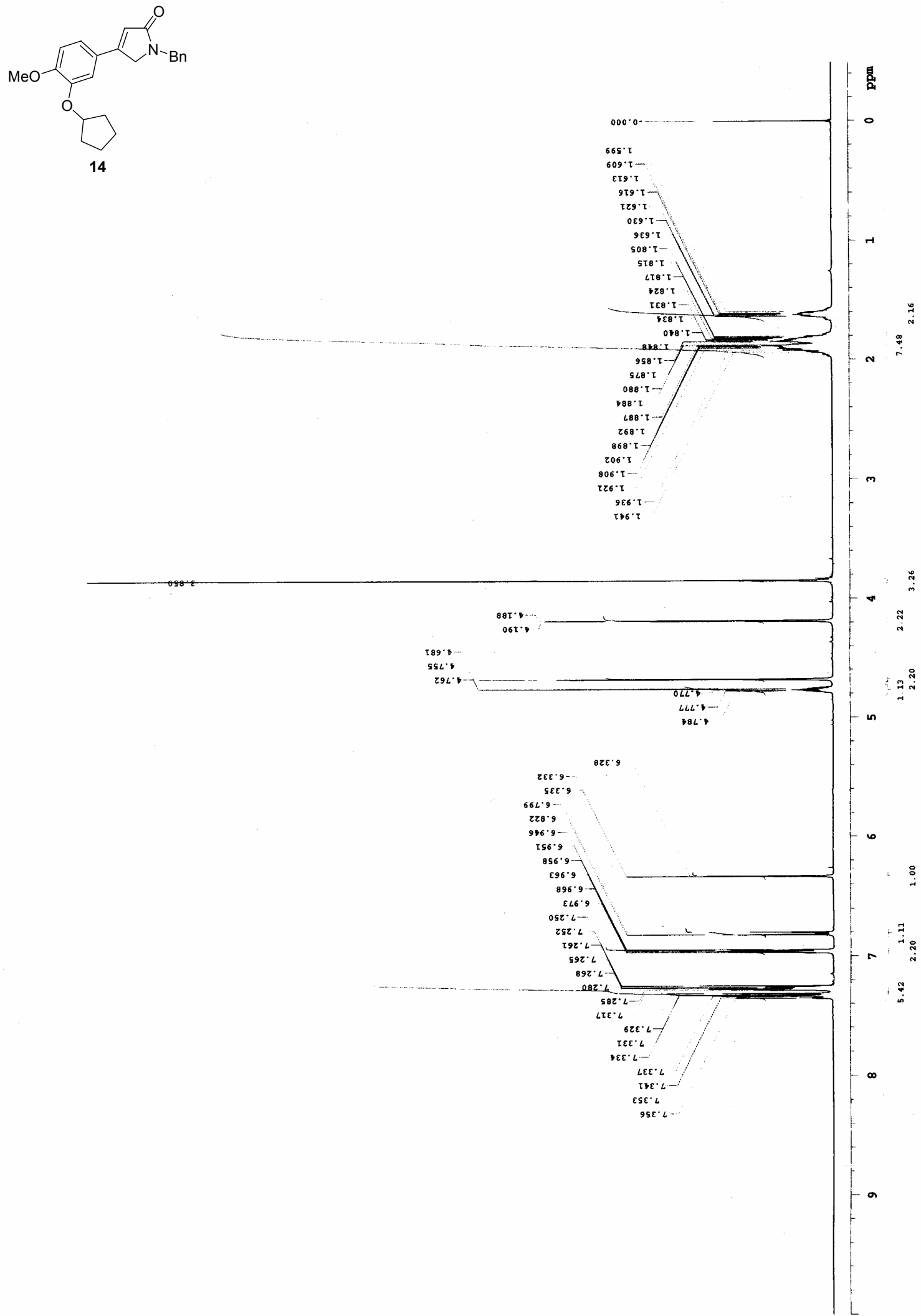
${ }^{13} \mathrm{C}$ NMR (100MHz, $\mathrm{CDCl}_{3}$ )
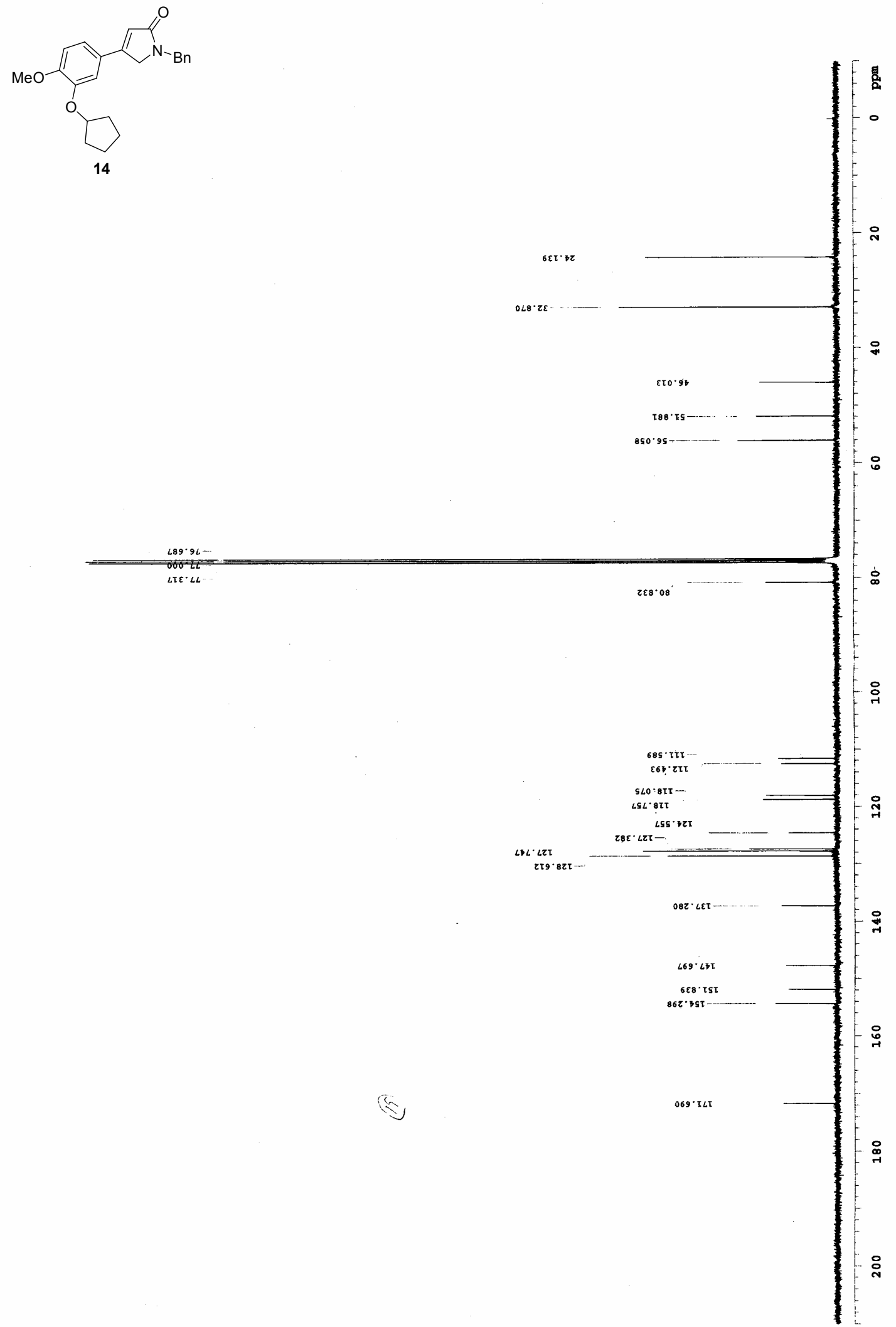


\section{${ }^{1} \mathrm{H}$ NMR $\left(400 \mathrm{MHz}, \mathrm{CDCl}_{3}\right)$}
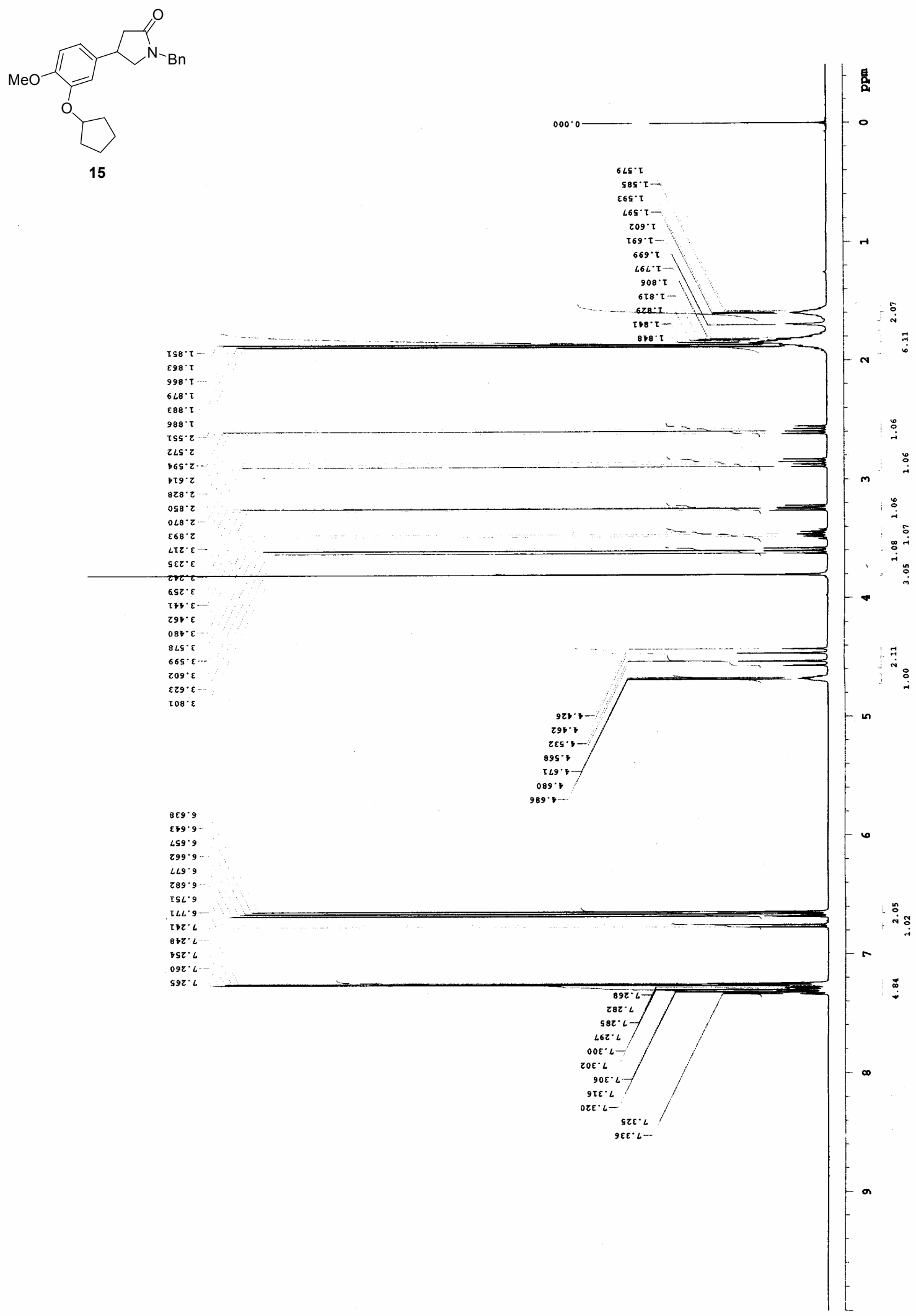
${ }^{13} \mathrm{C}$ NMR (100MHz, $\mathrm{CDCl}_{3}$ )
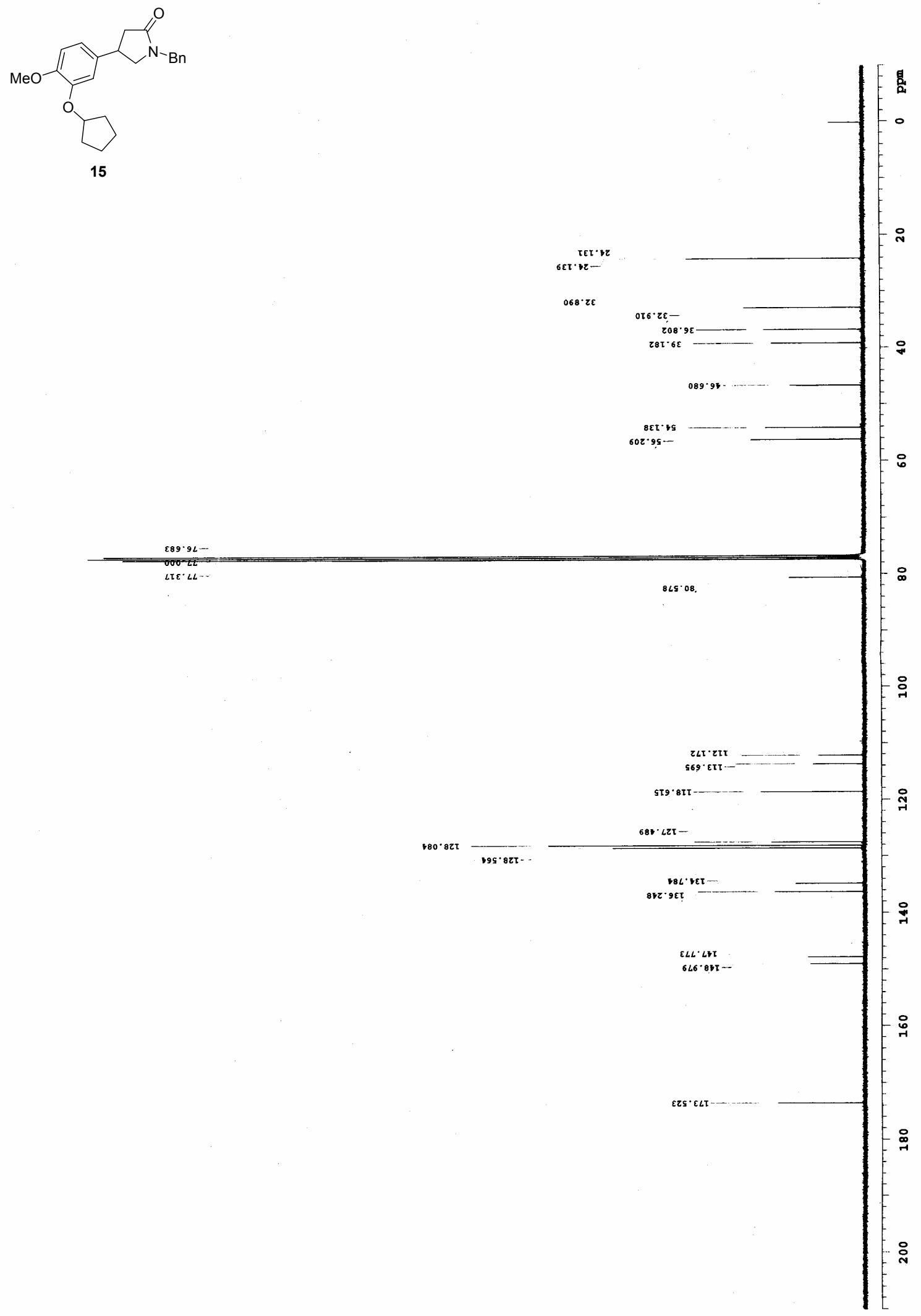
${ }^{1} \mathrm{H}$ NMR (400MHz, $\left.\mathrm{CDCl}_{3}\right)$

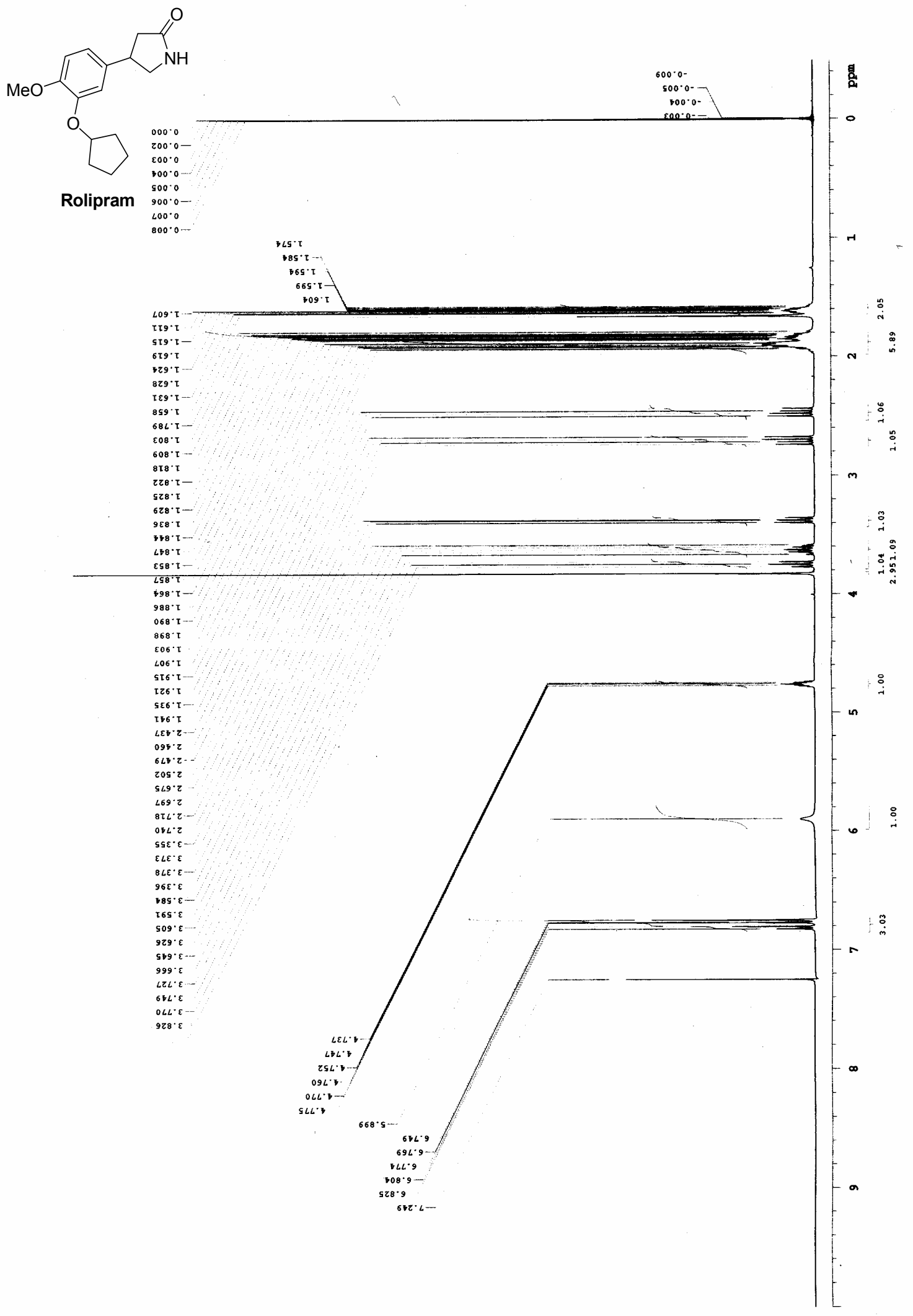


${ }^{13} \mathrm{C} \mathrm{NMR}\left(100 \mathrm{MHz}, \mathrm{CDCl}_{3}\right)$

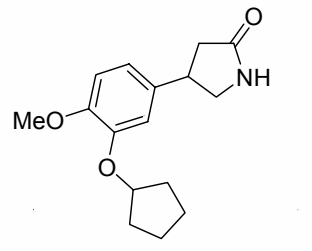

Rolipram

$\angle 89 \cdot 9 L-$

$90 z^{\circ} \angle L$

$2 \cdot L L \cdot$
$E \cdot L L$ 